Portland State University

PDXScholar

\title{
The Language of Transformation in a "Conversation for Possibility": A Metaphor Analysis
}

Deborah Jean Gabbert

Portland State University

Follow this and additional works at: https://pdxscholar.library.pdx.edu/open_access_etds

Part of the Philosophy of Language Commons

Let us know how access to this document benefits you.

\section{Recommended Citation}

Gabbert, Deborah Jean, "The Language of Transformation in a "Conversation for Possibility": A Metaphor Analysis" (1997). Dissertations and Theses. Paper 5486.

https://doi.org/10.15760/etd.7351

This Thesis is brought to you for free and open access. It has been accepted for inclusion in Dissertations and Theses by an authorized administrator of PDXScholar. Please contact us if we can make this document more accessible: pdxscholar@pdx.edu. 
THESIS APPROVAL

The abstract and thesis of Deborah Jean Gabbert for the Master of Science in Speech Communication were presented June 11, 1997, and accepted by the thesis committee and the department.

COMMITTEE APPROVALS:

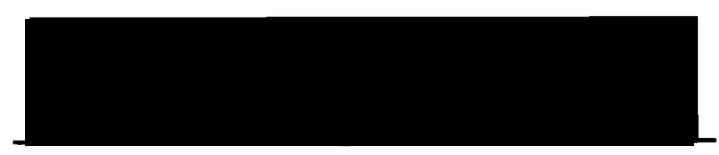

Susan B. Poulsen, Chair

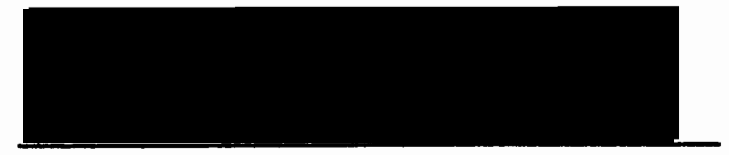

Peter Ehrenhaus

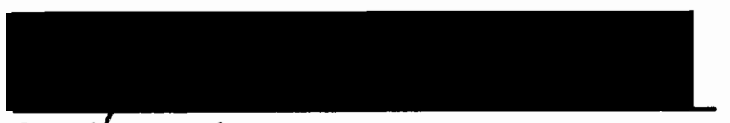

Stephen Reder

Representative of the Office of Graduate Studies

DEPARTMENT APPROVAL:

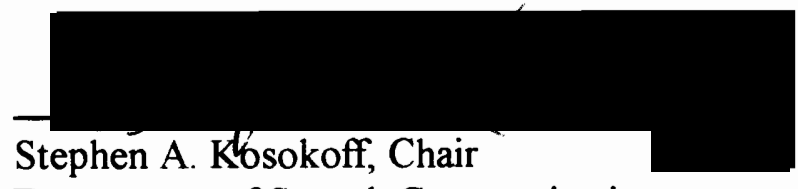

Department of Speech Communication

\section{ACCEPTED FOR PORTLAND STATE UNIVERSITY BY THE LIBRARY}
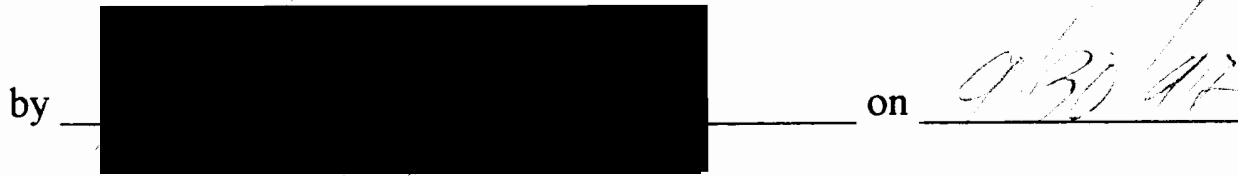


\section{ABSTRACT}

An abstract of the thesis of Deborah Jean Gabbert for the Master of Science in Speech Communication presented June 11, 1997

Title: The Language of Transformation in a "Conversation for Possibility": A Metaphor Analysis.

Metaphor allows us to understand and experience "relatively abstract or unstructured subject matter in terms of more concrete, or at least more highly structured subject matter" (Lakoff, 1993, p. 245). Lakoff and Johnson's (1980) and Lakoff's (1993) contemporary theory of metaphor contends that: metaphor is fundamentally conceptual; metaphoric structuring is the basis for the organization and functioning of much of the ordinary human conceptual system; and metaphorical language found in everyday speech is a surface manifestation of underlying conceptual metaphors. The metaphors most salient in a culture's discourse will reveal something about what is thinkable, knowable, and doable in that culture (Deetz, 1984). Metaphors have the power to create a perceptual shift, making possible new ways of thinking and acting that could create and be experienced as a paradigm-shift or transformation (Lakoff \& Johnson, 1980; Sackmann, 1989).

The present study investigated the intersection of transformation and culture using metaphor analysis to reveal and examine metaphors associated with Landmark Education Corporation (LEC). LEC is a training and development organization whose courses appear to be the catalyst for an experience of "transformation" and 
empowerment, as well as the core of a unique community of speakers.

"Texts" for the abstraction and interpretation of metaphors consisted of five in-depth interviews with LEC participants, participant notes from eight LEC courses, organizational artifacts, and field notes.

Interpretation and comprehension of emerging metaphors required a detailed explication of LEC's worldview, lending support to the idea that metaphors are indeed manifestations of how a culture thinks (Deetz, 1984). Analysis revealed a triad of root-metaphors (LIFE IS EMPTY AND MEANINGLESS, HUMAN BEINGS ARE MEANING-MAKING MACHINES, and LIFE IS A CONVERSATION) and four sub-systems of metaphors structuring: (1) language and conversation; (2) human "being"; (3) transformation; and (4) spatio-temporal relationships and values.

LEC's metaphoric system emphasizes "conversation" as creation, frames all modes of human "being" as products of this creation, and appears to facilitate empowerment by locating power within the individual and the individual within community. This study especially furthered understanding of the cultural embeddedness of metaphor and issues of cross-cultural interpretation. 
THE LANGUAGE OF TRANSFORMATION IN

A "CONVERSATION FOR POSSIBILITY":

A METAPHOR ANALYSIS

by

DEBORAH JEAN GABBERT

a thesis submitted in partial fulfillment of the

requirements for the degree of

\author{
MASTER OF SCIENCE \\ in \\ SPEECH COMMUNICATION
}

Portland State University

1997 


\section{ACKNOWLEDGMENTS}

Completing this project would simply not have been possible, let alone bearable, without the support of more people than I have the space, time, or energy left to specifically acknowledge. There are a few whose contributions (and suffering!) require special recognition:

My husband, Mark Bushbeck, who not only kept a roof over my head when the sky was falling (both metaphorically and literally!), but managed to love me when I was being unlovable, believe in me when I gave him no reason to, and allowed me to do it my way, even when my way seemed hopelessly "wrong." Like a true coach, Mark stood for who I am as a possibility and called me to be powerful.

Amanda Feller and Randy Baker, who provided shelter, inspiration, understanding, reality checks, moonlit walks, companionship, creativity, wisdom, hugs and stuff. Amanda and Randy are my soul-family, my spirit-guides on earth and my best friends.

Dr. Sue Poulsen, who was much more than the Chair of my thesis committee. Her unwavering patience, unswerving commitment, unyielding fortitude and unprecedented mentorship qualify her for sainthood, as well as gratitude, respect and admiration.

Franki Trujillo-Dalbey, Kathy Drew, Ruby Chen, Nobuko Higashi, Lyn Tan and Doreen Gilliam, whose generous listening, brilliant insights, and soulful camaraderie made the process stimulating and a lot less lonely.

My family and friends who understood my absences, didn't make me feel guilty when I procrastinated just a little and always encouraged me (to hurry up and get done so we could have some fun!).

The men and women whose time and sharing in the interviews was a priceless gift.

Thank you all. May the Metaphorce be with you! 


\section{TABLE OF CONTENTS}

PAGE

ACKNOWLEDGMENTS ................. . . . .

\section{CHAPTER}

1 INTRODUCTION . . . . . . . . . . . . . 1

2 REVIEW OF LITERATURE . . . . . . . . . . . . . 15

Metaphor, Thought \& Reality . . . . . . . . . . . . 15

Key Concepts . . . . . . . . . . . . . . . . 22

Metaphors \& Organizational Research . . . . . . . . . . . . . 29

Metaphors, Culture \& Issues of Communication . . . . . . . . 34

Descriptive Framework . . . . . . . . . . . 41

Ethnography of Communication . . . . . . . . 44 42

Empowerment, Transformation \& Community . . . . . . . 46

Social Construction of Reality . . . . . . . . . . . . 53

3 RESEARCH METHODS . . . . . . . . . . . . . 58

Qualitative Approach . . . . . . . . . . . . 58

Data Collection . . . . . . . . . . . . . . . . 59

Reliability \& Validity. . . . . . . . . . . . . . . . 68

Pilot Study. . . . . . . . . . . . . . . . . . . 74

Data Analysis . . . . . . . . . . . . . . . 75 
Metaphors \& Worldviews . . . . . . . . . . . . . 81

LEC's Worldview . . . . . . . . . . . . . . . . . 87

An Applied Ontology . . . . . . . . . . . . . . . . 87

The Nature of the World . . . . . . . . . . . . . . . . 89

The Individual's Place in the World . . . . . . . . . . . 95

The Range of Possible Relationships . . . . . . . . . . 105

5 FINDINGS--LEC'S EMERGENT SYSTEM OF METAPHORS . . 129

LEC's Root-Metaphors . . . . . . . . . . . . . . . . 129

LEC's Emergent System . . . . . . . . . . . . . . . . 139

Conversation . . . . . . . . . . . . . . . . . . 139

The "Being" in Human Being . . . . . . . . . . . . . . 159

Transformation . . . . . . . . . . . . . . . 174

Orientational Metaphor . . . . . . . . . . . . . . . 193

6 DISCUSSION \& CONCLUSION . . . . . . . . . . . . 206

Discussion of Findings . . . . . . . . . . . . . 206

A Critical Look at the Emergent System . . . . . . . . . . . 223

Limitations. . . . . . . . . . . . . . . . . . 232

Implications for Future Research. . . . . . . . . . . . . 234

Conclusion. . . . . . . . . . . . . . 236

REFERENCES . . . . . . . . . . . . . . 235 


\section{APPENDICES}

Appendix A . . . . . . . . . . . LEC Proposal Letter

Appendix B

Access Agreement

Appendix $\mathrm{C}-1 \& \mathrm{C}-2 \ldots \ldots$. . . . . Data Tables

Appendix D . . . . . . . . . . . . . . Informed Consent

Appendix E . . . . . . . . . . Interview Guide

Appendix F . . . . . . . . . Additional sources for information about Erhard, EST, and LEC 


\section{CHAPTER ONE}

\section{Introduction}

Lakoff and Johnson (1980), in their book Metaphors We Live By, build a compelling argument in support of their claim that the conceptual system giving rise to human thought and action, the conceptual system defining the everyday realities we experience, is "fundamentally metaphorical in nature . . human thought processes are largely metaphorical" (p. 3;6). This approach to metaphor is based on the assumption that ". . much of our ordinary conceptual system and the bulk of our everyday conventional language is structured and understood primarily in metaphorical terms" (p. 286). In other words, the way we think--the system of concepts giving rise to our thoughts--is fundamentally metaphorical in nature and this metaphoric nature is reflected in our daily talk:

Since communication is based on the same conceptual system that we use in thinking and acting, language is an important source of evidence for what that system is like. . . . Metaphors as linguistic expressions are possible precisely because there are metaphors in a person's conceptual system. . . . Since metaphorical expressions in our language are tied to metaphorical concepts in a systematic way, we can use metaphorical linguistic expressions to study the metaphorical nature of our activities. (pp. 3, 6-7)

Thus, metaphor is much more than a linguistic device, more than empirical verbal behavior or a "material manifestation" of a culture (Deetz, 1984). Metaphors not only describe a world in particular ways, they are the ways things are experienced in a world. The metaphors that are most salient in a culture will reveal something about what is thinkable, knowable, and doable in that culture: 
Metaphors are not lexical items that exist independently in a culture. Thinking, experiencing, and acting in a culture are based upon grand systems of metaphors. ... The patterns of metaphors, the system of similarities they entail, all trace the way a culture thinks" (Deetz, 1984, p. 218; 220).

Metaphors act as guides for human action and when our actions fit a metaphor, we reinforce the power of the metaphor to make experience coherent (Lakoff \& Johnson, 1980). Viewing metaphors in this way we begin to see their power to create, express and provide insight into individual and social realities.

The relationships among conceptual systems, metaphor, thought, and action imply that significant changes in the way an individual thinks and behaves are accompanied by changes in an individual's conceptual system, and thus, by implication, changes in the metaphors they live by. An experience that might create such significant changes, Deetz (1984) suggests, is learning to think, speak, and act within the context of a particular culture:

The learning of a language is central to acculturation, particularly as an adult moves from one culture to another. One of the ways a person comes to think in terms of another culture is through an adoption of their metaphors. As she or he learns these metaphors, she or he is learning more than particular metaphors ... she or he is acquiring an entire orientation to a set of problems. In doing so, experience comes to be structured in a new way. (p. 221)

If indeed metaphorical concepts infuse the very fabric of human thought and action, then it follows that the introduction of a new metaphor or system of metaphors into one's conceptual system could have the power to redefine one's reality, and hence, the nature of thought and action: 
New metaphors have the power to create a new reality. This begins to happen when we start to comprehend our experience in terms of a metaphor, and it becomes a deeper reality when we begin to act in terms of it. If a new metaphor enters the conceptual system that we base our actions on, it will alter that conceptual system and the perceptions and actions that the system gives rise to. (Lakoff \& Johnson, 1980, p. 145)

An appropriate word to label the experience described above might be

"transformation." A transformation can be defined as an experience that results in significant alterations in the ways people think, behave, feel, and relate to one another; an experience that changes perspectives, basic assumptions, and arguably, paradigms (Sackmann, 1989). Metaphors have a unique ability to trigger a perceptual shift, and, based on the preceding discussion, they may also provide an access for understanding the context and content of that shift.

\section{Study Purpose}

The purpose of this study was to investigate the intersection of metaphor, culture and transformation. This purpose was carried out using metaphor analysis to reveal and examine the meaning structures occurring within the cultural context of Landmark Education Corporation (LEC), a training and development organization whose courses appear to be both the catalyst for the experience of "transformation" and the core of a unique community of speakers.

Landmark Education Corporation (hereafter referred to as LEC) provides a curriculum of courses, beginning with a course called "The Forum," designed specifically to bring about "transformation" and create what might be called a 
"paradigm-shift"--an alteration in the way human beings conceive of and operate in

the world:

Its like, if you didn't know you were in that paradigm then you wouldn't ever sit and take a look at that or study that or think about it. Or question it or--because you didn't even know you were like that. ... [I]ts not just a concept, that is actually how the world is. That is your reality ... A paradigm is a reality. . so when you have the opportunity for a new reality, that's really powerful because it opens up all these new choices and possibilities that weren't there. They couldn't exist in the paradigm you lived in before. . . and you're not trying to change it, you're . . just shifting what is real for you. That's really powerful.(Jenna, 653-682)

A paradigm defines the limits of what we see, how we see, and the things we hold to be true. LEC claims that when we have the ability to identify the paradigms we live in and our own role in constructing and maintaining those paradigms, we can create whole new possibilities for living:

there is a shift in your thinking about other people and it is a transformation, I mean that's a word for it, because it really does alter your thinking about your relationships with other people. The way other people occur to you is different after the Forum ... that is certainly a transformation. . . . there can be incredible shifts in relationships just by a mere conversation. . . they call it the paradigmshift. . . that's exactly what happens. (Simon, 1199-1223)

Embedded in LEC's training content and expressed in the discourse of participants is evidence of a unique system of metaphors. Metaphors can be thought of as linguistic frames that capture or express cohesive structures of meaning that comprise a paradigmatic worldview (Smith \& Eisenberg, 1987). If, as Lakoff and Johnson suggest, metaphors in our speech are made possible by metaphors in our conceptual system, than studying the metaphorical structures in the discourse of a culture may 
provide unique insight into the shared reality or worldview of that culture.

\section{$\underline{\text { Cultural Context }}$}

The focus of this study is not the culture of the organization known as Landmark Education Corporation, but the cultural context shared by those who have participated in the courses that LEC offers. One might call this the "culture of participation." There are at least three practical reasons for making this distinction: (1) limitations in access to information (to be discussed in chapter three); (2) this researcher's primary interest in beginning to describe and understand the existing metaphoric system and the meaning participants make of this system, rather than critique the producers and production of that system; and (3) a need to maintain a manageable analytic scope.

The distinction between LEC's organizational culture and the "culture of participation" is admittedly artificial and problematic. Clearly the history, structure, goals, practices and symbolic meanings of Landmark Education as an organizational entity influence and are influenced by the system of meanings and experiences within the "culture of participation." Indeed, the cultural context shared by participants would not exist without the organizational entity that develops and delivers the curriculum, and with whom these individuals must interact if they wish to continue participating in LEC's courses.

Having acknowledged the artificial and problematic nature of the parameters adopted in this study, one must approach its findings and conclusions as a particular 
snapshot of the overall epic motion picture of LEC's culture. The brief sketch provided below is intended to introduce some of the relevant organizational aspects and issues associated with Landmark Education Corporation. This discussion is supplemented by an initial list of additional sources about LEC in Appendix F.

\section{Landmark Education Corporation}

LEC is an employee-owned, for-profit, training and development organization offering educational programs and curricula to individuals, organizations, communities, and institutions in more than 100 cities through 40 offices worldwide. LEC's "core" curriculum--"The Curriculum for Living"--consists of four distinct but interrelated programs designed to address issues in a developmental fashion, similar to the way conventional educational programs organize a curriculum of core courses sequentially to build students' competencies in key areas. The focus in the sequence moves from considering more individual issues to consider those of the individual participating in a network of social relationships and communities.

The four courses that make up the Curriculum for Living are The Landmark Forum (normally scheduled over three full days and one evening; tuition is $\$ 325$ ), The Forum in Action Seminar (an eight-session evening seminar series included in the cost of The Forum), The Advanced Course (scheduled over four full days and one evening; tuition is $\$ 700$ ), and The Self-Expression and Leadership Program (a comprehensive program meeting over a three-four month period with 10 weekly evening sessions and three full-day Saturdays sessions; tuition is $\$ 150$ ). The 
Landmark Forum is perhaps the most well known course in the Curriculum for Living; it the prerequisite course for participation in all other LEC programs and the one experience that all in LEC's speech community have in common. According to LEC, the Curriculum for Living is designed to:

[C]hallenge conventional thinking and perspectives, and provide new paradigms, opportunities, and tools--even use a new language--for effecting significant change and shifting the very nature of what is possible in people's lives, in their organizations, their community groups, and their institutions. (LEC Memo, 1993, p. 1)

In addition to the Curriculum for Living, LEC offers a Graduate Curriculum of approximately 30 workshops, seminars, and programs designed to explore specific areas such as communication, leadership, accomplishment, and creativity. According to a 1994 LEC News Release, approximately 60,000 people a year participate in the Curriculum for Living worldwide. Approximately 50,000 people participate in these"graduate" course offerings yearly (1994 LEC News Release). LEC also offers a Forum for Young People (ages 8-12 years) and a Forum for Teens (13-18 years).

Two courses in the Curriculum--The Forum and The Advanced Course--are led exclusively by a member of the "Forum Leader Body." This "body" is comprised of 44 men and women who must complete a rigorous, full-time training and apprenticeship commitment (5-7 years to complete depending on individual progress) before they can actually lead the Forum. The Teen and Young People's Forums are lead by members of the Forum Leader body specifically trained to work with youth. The remaining course offerings are lead by "seminar leaders" or "course 
leaders": volunteers who, after completing the Curriculum, spend several months or years training and developing themselves as program leaders.

All Landmark programs, as well as day to day operations of the organization, are dependent upon the substantial assistance of volunteers, all of whom are at least graduates of the Forum. Volunteer participation in LEC is called "assisting." Those who assist are part of the "assisting body" participating in the "Assisting Program." Assisting opportunities vary widely from aspects of course coordination and management to working in the Landmark offices answering phones, performing data entry, and other office related work. Only Forum Leaders and staff/employee positions are paid.

LEC was officially incorporated in January of 1991 in the State of California. LEC is a for-profit, employee owned company, with no employee owning more than two percent of stock in the company. Those who leave their employee positions sell their stock back to LEC. The management of LEC reports to a Board of Directors elected by the employee stockholders.

Origins and historical context. LEC's organizational initiatives and educational offerings evolved from the research and technology of Werner Erhard's controversial EST (Erhard Seminars Training) programs that began in the early 1970's. This evolution entailed comprehensive redesigning of Erhard's methods and materials into the programs that are today offered by Landmark Education. During the 1980's, Werner Erhard began disengaging himself from the work and the EST 
programs were permanently retired. By 1991, Erhard had licensed the totality of the program materials to LEC, giving up all rights of ownership. According to Landmark, Erhard has not been involved with the organization in any significant influential capacity, financial or otherwise, since that time.

The controversy attached to Erhard and EST involves a number of different dimensions. For example, Erhard himself was the focus of considerable media controversy in relationship to alleged financial and personal/family scandals (Self, 1992). Both EST and LEC have each received critical attention regarding various organizational procedures, policies and presentational methods (e.g. sales techniques, potential conflict between being for-profit and fulfilling educational /service goals, methods of influence used, etc.) (Jackson, 1996). Much of the negative publicity regarding Erhard and EST has been attributed to a campaign waged by the Church of Scientology to discredit Erhard "by any means necessary" (Welkos, 1991). The pejorative labeling of EST, and currently Landmark, as a "cult" has also been the subject of a variety of articles and letters--even a lawsuit against the Cult Awareness Network (CAN) for its public listing (which was later retracted by them) of LEC as a cult (Self, 1992).

I will not undertake an extensive historical explication or a comprehensive survey of relevant controversial issues in this thesis. However, I strongly recommend that interested readers consult the numerous sources available to more thoroughly explore the historical and controversial aspects of EST and LEC. One 
good starting place is an internet site titled "Landmark Education Corporation and the Forum: Rants and Raves" (http://www.inlink.com/ dhchase/forum.htm\#top). From this site one can access bibliographies of published books and articles, relevant news groups and internet links providing a full-range of perspectives and experiences regarding LEC. Several potential sources have been referenced in the preceding three paragraphs and several additional suggestions can be found in Appendix F.

Participation. Those who choose to participate in LEC come from diverse backgrounds in age, education, career, interests, socio-economic status, religious and spiritual commitments and ethnic heritage. The "average" participant is between the ages of $25-44(65 \%)$; with at least some college education (59\%) and careers that fall within the categories of "professional/technical," "manager/self-employed," and "sales" (72\%) (Sharing the Forum brochure, 1995).

For those who continue their association with LEC after the Forum, participation tends to take on the quality of participating in a "community." The community aspect that emanates out of LEC's programs can be attributed to a variety of components, although the experience of community can not be adequately accounted for as a sum of these parts.

One factor is that everyone who participates in LEC has completed the Forum which creates a base of shared meanings and experiences, whether or not people were in the same Forum. The Forum itself is a mentally, emotionally and physically 
intense experience involving three consecutive 12-16 hour days of participatory inquiry, dialogue, and "coaching"; enormous amounts of individual sharing of stories, insights, challenges, and a variety of interactive and individual exercises and assignments designed to facilitate the goals of the course.

Shared experiences, a sense of connection and acceptance, active participation, emotional release, cognitive stimulation, new language and ideas, and an opportunity for ongoing association--all these contribute to, but do not explain, the creation of community. The goals of this study do not include or hinge upon accounting for the creation of this community. What is important is that LEC is both an organization and the source of an active and distinct community.

My Involvement. My scholarly interest in LEC emerged out of my personal experiences as a participant in their course offerings. I completed the Forum in November of 1994 and went on to participate in the rest of the Curriculum for Living (the Advanced Course (AC), Forum in Action seminar (FIA), SelfExpression and Leadership program (SELP) and several "graduate" offerings: the Communication: Access to Power program (CAP), Breakdown seminar (Brkdwn), and Relationship seminar (Relshp). I have also participated with LEC in various assisting capacities, including a three month agreement to provide one-on-one "coaching" to a group of participants working on community projects in the SELP program.

My initial interests revolved around four inter-related themes. First is the 
observation of a unique vocabulary and "way of speaking" that appears to be integral to both the process and product of transformation. This "way of speaking" is introduced in the Forum and developed in subsequent courses as participants learn "how to speak the language of transformation" (pilot log, p. 8, 19). Second, LEC places an extraordinary and refreshing emphasis on the centrality of language and communication in creating the realities we experience. Everything, according to Landmark, is a function of communication. Third, I have observed a powerful climate of self-in-control and being-in-action that seems to manifest in people's lives as a result of their participation in LEC. Individuals develop (or discover) an ability and desire to take responsibility for and responsible action in creating their lives. And finally, I have mentioned the appearance of a definable community or coculture as an emergent property of participation in LEC's courses.

The overall purpose of this study is to contribute to our understanding of the ways in which metaphors both create and express individual and social realities by exploring the system of metaphors within LEC's "culture of participation." Metaphor analysis is an effective approach for providing access to the "deep" meanings of a culture and insight into the patterns of thinking, being, and acting that characterize a cultural system (Koch \& Deetz, 1981; Deetz, 1984).

\section{$\underline{\text { Research Questions }}$}

The following research questions guided this study: 
1. What constitutes the metaphorical system embedded in and abstractable from various LEC texts?

2. What is the experiential structure provided by LEC's metaphoric system?

3. How do metaphors and their experiential structure manifest in the discourse and lived experiences of those who participate? How do LEC participants make metaphors meaningful in their everyday lives?

4. What are the meaning structures associated with people's experiences of transformation, empowerment, and community?

\section{$\underline{\text { Research Goals }}$}

The process of answering the research questions posed above will provide more than insight into an alternative culture and reality. Fulfilling the purpose of this study also provides an opportunity to examine our taken-for-granted metaphors as we contrast what we know with what is unfamiliar. As we seek to better understand others, we create the possibility of seeing our own lives in new ways.

I say that interpretation is the process by which disclosure of new modes of being ... gives to the subject a new capacity for knowing himself. If the reference of the text is the project of the world, then it is not the reader who primarily projects himself. The reader rather is enlarged in his capacity of selfprojection by receiving a new mode of being from the text itself. (Ricoeur, 1976. p. 94).

\section{Chapter Summary}

The preceding chapter introduced Lakoff and Johnson's (1980) theory that metaphor is responsible for structuring much of the human conceptual system and that metaphors in our speaking are evidence of that conceptual structuring. Metaphors are embedded in cultural systems, shaping and reflecting the way a culture experiences a reality. The possibility that new metaphors might create and be 
experienced as a transformation was explored. Landmark Education Corporation, its courses, participants and unique cultural context was discussed in relationship to the purposes and questions of this study.

Chapter two presents the literature review and theoretical framework that informs this study. Chapter three presents the research methods used to carry out the study. Both chapter four and five present the findings of this study: chapter four explicates LEC's worldview and chapter five presents the emergent system of metaphors associated with that worldview. Chapter six provides a final discussion of and conclusions about the findings. 


\section{CHAPTER TWO}

\section{Theoretical Framework and Literature Review}

Metaphor, Thought \& Reality

The primary source of theoretical influence in this study is that of the symbolic and formative significance of metaphor in shaping and representing individual and social of social realities. Essentially, metaphor allows us to understand and experience "relatively abstract or unstructured subject matter in terms of a more concrete, or at least more highly structured subject matter (Lakoff, 1993, p. 245). (e.g. understanding A as B; understanding the internet as "a highway"). Lakoff and Johnson's (1980) and Lakoff's (1993) contemporary theory of metaphor contends that metaphor is fundamentally conceptual, that metaphoric structuring is the basis for the organization and functioning of much of the ordinary human conceptual system, that metaphor is "the main mechanism through which we comprehend abstract concepts and perform abstract reasoning" (Lakoff, 1993, p. 244), and that metaphorical language found in everyday speech is a surface manifestation of underlying conceptual metaphors.

We are largely unconscious of how language generally, and metaphor specifically, shapes our experience and actions. Much of the human conceptual system is structured by conventional metaphors that have become fixed, sedimented over time through habitual use, and no longer recognized as metaphors. These conventional metaphors form our everyday ways of conceptualizing experience and 
find expression in our everyday discourse. For example, the conventional metaphor TIME IS A VALUABLE COMMODITY sanctions expressions such as "Quit wasting time" and "That was worth the time I spent on it." Conventional conceptual metaphors such as this occur as literal, but are still based on mapping two different domains of experience onto one another, functioning "automatically," "below the level of consciousness," with "no noticeable effort" as it shapes both our "our understanding of experience and the way we act on that understanding" (Lakoff, 1993, p. 245).

In all aspects of life. . we define our reality in terms of metaphors and then proceed to act on the basis of the metaphors. We draw inferences, set goals, make commitments, execute plans all on the basis of how we in part structure our experience, consciously and unconsciously, by means of metaphor (Lakoff \& Johnson, 1980, p. 158).

Think of the metaphors we use to understand and talk about our experience of life:

life is a game, life is a journey, life is hard, life is a stage, life is a roller-coaster ride. Metaphoric entailments associated with a particular metaphorical concept highlight and make coherent certain aspects of our experience, allowing us to understand and experience one kind of thing or domain of experience in terms of another. These same entailments that create understanding through highlighting also downplay or obscure other aspects of experience that are inconsistent with that metaphor (Lakoff \& Johnson, 1980).

Consider for a moment what type of entailments follow from the metaphor "life is a game." Life as a game involves strategies, moves, rules, winners and losers, 
competition, chance, skill, discernable beginnings and definite outcomes, etc.

Viewing the world through the metaphor LIFE IS A GAME hides aspects of life that may be inconsistent with our understanding of games such as process, destiny, harmony, or cooperation. The systematic set of entailments of a game provide us with a particular way of conceptualizing the experience of life and our concepts have important implications for how we structure our experiences and enact our responses in the world.

New metaphors, like conventional metaphors, highlight and downplay particular aspects of experience through their systems of entailments. They can provide a unique perspective about a domain of experience and thus, make possible new ways of thinking and acting. Consider the recent popularity of the INFORMATION HIGHWAY metaphor after Vice President Gore's 1993 speech:

Today, commerce rolls not just on asphalt highways, but along information highways. . . think of the National Information Infrastructure as a network of highway . . . and I'm not talking about just one eight-lane turnpike. I mean a collection of Interstates and feeder roads made up of different materials in the same way that roads can be concrete or macadam--or gravel. Some highways will be made up of fiber optics. Others will be built out of coaxial or wireless. (cited in Rohrer, 1995)

The "highway" metaphor itself was not a novel one, but the rich domain of experiences contemporary industrialized cultures like the U.S. have with highways allowed it to be extended in a variety of novel ways to provide new understandings of our experiences, as well as providing a basis for a variety of arguments and social policy initiatives regarding internet technology. Further examples of such novel 
extensions can be seen in some quotes from contemporary news reports and headlines: "White House counts two million cybertourists," AT\&T stalled on the info-highway," and "Potholes along the information highway--if this is the highway, I'd rather hitchhike on a country road" (cited in Rohrer, 1995). Accepting a new metaphor, such as THE INFORMATION HIGHWAY, and reinforcing it with action appropriate to the metaphor (passing social policy for example) may lead us to view the metaphor as true (Lakoff \& Johnson, 1980).

This cognitive approach to metaphor, unlike others, puts metaphor in the driver's seat, so to speak. Language, thought, and hence action, are all metaphorically structured, not just metaphorically expressed or influenced. Other scholars have duly noted the power of metaphor in creating and communicating meaning, shaping and reflecting our realities, although there is still disagreement regarding just how metaphor works and what it is for.

Black (1962, 1993), discusses the distinction between substitution, comparison, and interaction views of metaphor. The substitution view of metaphor is one in which a metaphorical expression is viewed as being "used in place of some equivalent literal expression," either to remedy some gap in the our literal vocabulary or as a stylistic move for the purposes of aesthetics (1962, p. 31). Either purpose, says Black (1962), constitutes metaphor as a deviation.

The comparison view of metaphor is characterized by "the presentation of the underlying analogy or similarity" between the literal and figurative, resulting in 
"some transformation of a literal meaning" (Black, 1962, p. 35). In this view, metaphor is different from simile or analogy only in the form of its expression, which implies rather than explicitly states the comparison. The similarity between the two parts of the metaphor is then, already present, and the metaphor an artful device for expressing it.

Neither of these views, according to Black, adequately accounts for the phenomenon of metaphor. Instead, he poses an interaction view of metaphor, the origins of which can be located in the work of Richards (1936). Black (1962) interprets Richards as saying that in metaphor, our thoughts regarding two different things interact or become "active together" "to produce meaning that is resultant of that interaction" (p. 38). In this way, both the tenor and the vehicle of the metaphor (or in Black's terms, the focus and the frame) acquire new meaning. In the example "the poor are the negroes of Europe," Black explains:

that in the given context the focal word "negroes" obtains a new meaning, which is not quite its meaning in literal uses, nor quite the meaning which any literal substitute would have. The new context (the "frame" of the metaphor in my terminology) imposes extension of meaning upon the focal word. . . f for the metaphor to work the reader must remain aware of the extension of meaning--must attend to both the old and the new meanings together. $(1962$, p. 39)

The system of associated commonplaces (otherwise known as entailments) of the frame of a metaphor acts to select, emphasize, suppress, and organize the features of the focus in a manner similar to a filter: "We can say that the principal subject is 'seen through' the metaphorical expression" (Black, 1962, p. 41). Black's 
(1993) view of metaphor would allow that they sometimes act as "cognitive instruments" in constituting and revealing a particular view of reality. Still, Black's interaction view of metaphor--even what he calls "strong" metaphor--maintains a primarily representational view of metaphor as a device of communication for conveying and creating meaning.

Lakoff and Johnson's (1980) theory of metaphor goes beyond the substitution, comparison and interactional views to argue that metaphor is inseparable from the way the human mind works; our use of metaphoric linguistic expressions becomes observable evidence for our cognitive structure and processes. How metaphor works and implications for how it is and can be used, become issues of central importance, beyond what they might represent or reveal about conceptions of reality.

Ortony (1993) suggests that contemporary scholars of metaphor "vary in their theoretical persuasions" regarding the significance and centrality of metaphor "almost along constructivist/nonconstructivist lines," representing different opinions about the relationship between language and the world (p. 3). Nonconstructivist positions tend to treat metaphors as deviant, ornamental, and figurative variations of "normal" literal language usage, requiring explanation as to how they violate linguistic rules. In this view, metaphoric language would be seen as providing a figurative rather than literal description of reality. The roots of the nonconstructivist positions can be traced back to Aristotle's original treatment of metaphor in the 
Poetics as a decorative, but deviant figure of speech that, when used sparingly, could add power to one's speech. In Aristotle's view, metaphors were implied comparisons derived from the logic of analogy, which in the contemporary study of metaphor is equivalent to the "comparison" theory of metaphor (Ortony, 1993). Richards (1936) comments on the historical trend of viewing metaphor as deviation from language's normal operation instead of its fundamental and constitutive form:

Throughout the history of Rhetoric, metaphor has been treated as a sort of happy extra trick with words, an opportunity to exploit the accidents of the versatility, something in place occasionally, but requiring unusual skill and caution. In brief, a grace of ornament or added power of language, not its constitutive form. (p. 90).

Richard's himself approached metaphor as "omnipresent" in language and constitutive in its functioning--thought itself, according to Richards, was metaphoric, proceeding by comparison, "and the metaphors of language derive therefrom" (p. 94). As such, Richard's work in metaphor is consistent with constructivist positions on metaphor.

Constructivist positions regard language use and comprehension--and thus, metaphor--as essentially creative activities in the construction of meaning (Ortony, 1993). On the constructivist side of the continuum, rhetorical scholars view metaphor as a basic symbol using process by which our knowledge and experience of reality occurs:

Underlying this view is the assumption that we cannot know reality in any objective way. We do not stand apart from the world and perceive reality and then interpret or give it meaning. Rather, we have or know a reality only 
through the language by which we describe it. We constitute reality through our use of symbols. (Foss, 1989, p. 188)

Metaphor from this view is significant in its power to make reality accessible to us: "what ever language we use to describe reality is a metaphor because it enables us to see reality as something" (Foss, 1989, p, 188). This conception of metaphor highlights its constitutive, epistemic and culturally-situated properties.

\section{$\underline{\text { Key Concepts }}$}

\section{Metaphorical Concepts and Metaphoric Linguistic Expressions}

Essentially metaphor allows us to understand and experience one, typically less structured kind of thing, in terms of another, typically more structured kind of thing. Lakoff and Johnson (1980) propose that we understand and define the world as domains of experience or experiential gestalts that "characterize structured wholes within recurrent human experiences" (p. 117). These domains are a product of our experiences with our bodies (perceptions, emotions, mental and motor capabilities), our interactions with the physical environment (moving, eating, manipulation of objects), our interactions with other people (in social, political, economic, and spiritual contexts). Lakoff(1993) tells us that we can understand metaphorical concepts as mappings from a "source-domain"--the domain of experience being used to organize--to the "target-domain"--the domain of experience one is attempting to structure. It is in the interaction (or the coherence) of the two domains--the tenor 
and the vehicle, the focus and the frame--that a metaphor organizes our understanding of one thing in terms of another.

The mapping of a metaphorical concept represents a set of ontological correspondences (Lakoff, 1993) or entailments. So, for example, in the metaphorical concept LIFE IS A GAME, the target-domain is "life" and the source-domain is "game." If I speak of the metaphorical concept LIFE IS A GAME, I am referring to a set of correspondences that characterize this concept or mapping. For example:

People you interact with correspond to players.

Actions following from decisions correspond to moves.

Forces impinging on actions correspond to rules.

Plans enacted to achieve specific outcomes correspond to strategies.

The mapping tells us how experience, in this case life, is being conceptualized:

The mapping is primary, in that it sanctions the use of source domain language and inference patterns for target domain concepts. The mapping is conventional, that is, it is a fixed part of our conceptual system, one of our conventional ways of conceptualizing [experience]. (Lakoff, 1993, p. 208)

In this way, the correspondences and inference patterns of the source-domain are mapped onto the target domain. Lakoff(1993) notes that contemporary researchers of metaphor use the term "metaphor" to indicate a metaphorical concept or mapping. Metaphorical concepts then, are realized and expressed in metaphoric linguistic expressions: "Metaphors as linguistic expressions are possible precisely because there are metaphors in a person's conceptual system" (Lakoff \& Johnson, 1980, p. 6). Possible metaphoric linguistic expressions generating from the metaphorical concept LIFE IS A GAME might be: 
"What's your next move?"

"It was obvious he had worked out a superior strategy when he out maneuvered me."

"Who are all the key players?"

As suggested by Lakoff and Johnson (1980) and Lakoff (1993), the word metaphor is used in this study to indicate a metaphorical concept or conceptual mapping, represented in the form of all caps, such as LIFE IS A GAME.

Mappings and their sets of correspondences can be referred to as domains of experience or experiential gestalts--multidimensional structured wholes whose various dimensions are defined in terms of concepts emerging naturally from our experience (Lakoff \& Johnson, 1980). In the LIFE IS A GAME example, the gestalt of GAME arises from our experience with games. We experience life as a game when we recognize that the GAME gestalt fits our perceptions and actions as we go about our daily lives.

In this way we classify particular experiences in terms of experiential gestalts in our conceptual system. . . It is by means of conceptualizing our experiences in this manner that we pick out the 'important' aspects of an experience. And by picking out what is 'important' in the experience, we can categorize the experience, understand it, remember it. (Lakoff \& Johnson, 1980, p. 83)

Classifying our experiences in this way is what tells us what to do--what action is called for, appropriate, inappropriate, expected, etc. Lakoff and Johnson thought it important to reinforce the distinction between

(1) the experience itself, as we structure it, and (2) the concepts that we employ in structuring it, that is, the multidimensional gestalts [or metaphorical concepts]. The concept [e.g. GAME] specifies certain natural dimensions [e.g. players, rules, winning/losing, etc.] and how these dimensions are related. There 
is correlation, dimension by dimension, between the concept [GAME] and the aspects of the actual activity [of playing a game]. This is what we mean when we say that a concept fits an experience. (p. 83)

\section{Structural Metaphor}

Structural metaphors operate much as the term suggests: they impose the structure and knowledge of one domain of experience (the source-domain) onto a less clearly delineated domain of experience (target-domain). It is through our knowledge of the source-domain that we structure and understand the targetdomain--we understand one concept within the structure or frame of another concept. Cultural context is a crucial component influencing a metaphorical concept's capacity to provide such understanding, since all cultures may not have the same understanding of or experience with the source-domain (Lakoff \& Johnson, 1980). For example, the INFORMATION HIGHWAY metaphor would not provide much in the way of useful source-domain experience in a culture that had nothing resembling a highway or did not value highways.

Metaphoric structuring, it should be noted, is partial: "If it were total, one concept would actually be the other, not merely understood in terms of it" (Lakoff \& Johnson, 1980, p. 13). This partial structuring of a concept will focus our attention on certain aspects while simultaneously preventing us from focusing on those aspects that are inconsistent with the metaphoric structure being imposed. Consider how the INTERNET AS INFORMATION HIGHWAY has seemed to eclipse the use of an earlier internet metaphor of "surfing." Surfing is a recreational 
frame; the goals appropriate to surfing (sport, adventure, leisure, skill, etc.) do not fit with the goals of commerce, speed, and progress that are part of the "information highway" structuring.

\section{Orientational Metaphor}

Orientational metaphors organize whole systems of concepts with respect to one another, rather than one concept in terms of another. Lakoff and Johnson focus primarily on spatial orientations such as up-down, in-out, front-back, on-off, deepshallow, etc. Orientational metaphors are grounded in the experience of our physical bodies functioning in our physical and cultural environment; they provide understanding by giving concepts spatial orientations derived from this physicalcultural experiential basis. Our physical and cultural experience provides a variety of possible bases for orientations. For example:

\section{HAPPY IS UP; SAD IS DOWN}

I'm feeling up. That boosted my spirits. My spirits rose. You're in high spirits. Thinking about her always gives me a lift. I'm feeling down. I'm depressed. He's really low these days. I fell into a depression. My spirits sank. (Lakoff \& Johnson, 1980, p. 15)

Possible physical bases for such a metaphor include: Drooping posture typically goes along with sadness and depression, erect posture with a positive emotional state; when people are happy the tend to smile and the smiling facial expression is up, where a frown, associated with sadness, is down. Orientational metaphors are not arbitrary. An overall system of orientational metaphors will exhibit external coherence (e.g. HAPPY IS UP is coherent with GOOD IS UP) as well as 
systematicity among the linguistic expressions associated with a metaphor (e.g. if "I am walking on cloud nine" meant "I am horribly depressed" it would be incoherent with HAPPY IS UP).

As reflected in the previous discussion, metaphors are culturally situated and thus, subject to and framed in cultural terms. Orientational metaphors tend to be among the most fundamental in a culture and thus, a culture's most fundamental values and the metaphorical structure embedded in the culture will be coherent with one another. In U.S. mainstream culture, for example, "up" is generally valued (e.g. "He moved rapidly up the corporate ladder"), unless of course we are talking about inflation. Lakoff and Johnson (1980) explain that prioritization of these kinds of variations might be based on which orientation has the clearest physical base. In this case, MORE IS UP has priority over GOOD IS UP because "more" has a stronger grounding in physical, concrete experience than "good" which is an abstract interpretation. Prioritization of orientational metaphors and the values they indicate also applies to subcultures and to "groups whose defining characteristic is that they share certain important values that conflict with those of the mainstream culture" (Lakoff \& Johnson, 1980, pp. 23-24), and to the value systems of individuals that may conflict with the metaphors of mainstream culture.

In general the major orientations up-down, in-out, central-peripheral, activepassive, etc., seem to cut across all cultures, but which concepts are oriented which way and which orientations are most important will vary from culture to culture. (Lakoff \& Johnson, 1980, p. 24). 
In other words, all cultures seem to recognize the major variations of spatiotemporal orientations humans share the experience of physical bodies in a physical environment. However, how these orientations are used to structure concepts and how they are prioritized is influenced by the unique experiences of a particular culture.

\section{Ontological Metaphor}

Ontological metaphors allow us to understand our experiences by partially structuring them in terms of entities or substances. In this way, we identify experiences that are not clearly discrete or bounded as physical objects or substances that are, thereby allowing us to

refer to them, categorize them, group them, and quantify them--and, by this means, reason about them. . . Human purposes typically require us to impose artificial boundaries that make physical phenomena discrete just as we are: entities bounded by a surface. (Lakoff \& Johnson, 1980, p. 25).

Like orientational metaphors, ontological metaphors are experientially based; in this case it is our experience with physical objects that gives rise to the metaphorical concepts. In most cases, ontological metaphors, like orientational metaphors, are not noticed as metaphorical, but as actual descriptions of phenomena. For example, expressions like: "He cracked under pressure" and "He broke down." are indicative of two common ontological metaphors in our culture that provide a model for understanding the mental phenomena: THE MIND IS A BRITTLE OBJECT and THE MIND IS A MACHINE. The expressions above occur as natural because 
these two metaphors are integral to our culture's understanding of the human mind.

Metaphoric linguistic expressions (e.g."We need wider highways to accommodate the information loads of cutting edge technologies") are generated from metaphorical concepts that map inference patterns from a source-domain (HIGHWAYS) to a target-domain (THE INTERNET). The categories of structural, orientational and ontological metaphors provide a useful heuristic tool for analyzing different types of metaphorically structured experience and examining the coherence among the metaphors comprising a system.

\section{Metaphors and Organizational Research}

This theoretical framework for approaching the study of metaphor is strengthened by studies focusing on uncovering and understanding the systems of thinking and action that characterize a particular organization. Three in particular are discussed here. The first is an explication of how to use Lakoff and Johnson's framework to uncover the social realities of an organization. The second uses the concept of a "root-metaphor" to study conflict within an organization The third explores the possibility for metaphors to trigger and facilitate processes of organizational transformation.

\section{Metaphor Analysis and Organizational Realities}

Koch and Deetz (1981) explained and illustrated the use of Lakoff and Johnson's (1980) theoretical framework as analytic tool for metaphor analysis in organizations: 
which accomplishes three interrelated tasks: (a) provides a description of the meaning structures in organizations which constrain discourse, (b) clarifies the meaning and effect on social reality of verbal expression in the organization, and (c) provides a basis for criticism of and intervention in the existing social reality of the organization. (pp. 1-2)

They argue that meaning structures comprising the social reality of an organization are produced and reproduced in members' metaphorical expressions. This proposed relationship between metaphorical expressions and social realities has powerful implications for the significance of metaphor as the "taken for granted 'deep' meaning that contextualizes action and understanding as organizational members go about their work":

When we say, then, that members of an organization share a social reality, we are not simply saying that they share a view of the world and a way of talking about it; more fundamentally they share a world. What is thinkable, doable (in terms of possibility), is neither a matter of choice nor persuasion but serves as a background from which all choice and persuasion emerge. (Koch \& Deetz, 1981, p. 3).

Koch and Deetz (1981) successfully illustrate how metaphor analysis can be used to isolate and illuminate the predominant metaphors and their entailments in order to describe the "current reality and conceptions" of the organizational members (p. 13). Exposing the current reality also reveals the possibility of other realities. Although Koch and Deetz focused on explicating an organizational reality, their method can be readily adapted to metaphor analysis within a particular cultural context (see pp. for an example of such an application). 


\section{Root-Metaphor Analysis}

A field study conducted by Smith and Eisenberg (1987) used metaphor analysis to expose fundamental differences in world view between management and employees of Disneyland, represented by conflicting "root-metaphors." A "rootmetaphor" is a conventionalized symbolic frame, the roots from which a particular worldview and an associated system of metaphorical concepts and metaphoric linguistic expressions "stems." One of the identifying characteristics of rootmetaphors is their "ability to undergird a broad area of meaning" capturing "a fundamental, underlying worldview" and providing "an inferential base for understanding more discrete attitudes and behavior" (Smith \& Eisenberg, 1987, p. 369).

In the case of Disneyland, extensive document analysis and a series of interviews with management and hourly employees revealed two primary rootmetaphors: the "Disney experience as drama" and "the Disney experience as family." The "drama" metaphor was evident in organizational talk and philosophy from the outset of Disneyland's creation. The "family" metaphor, while it seemed to exist during the early years, began to emerge with a new vitality after the loss of Disney's founder resulted in management attempting to cultivate the idea of a "Disney philosophy" to embody the spirit of Walt Disney. Both employees and management seemed to adopt the "family" metaphor, but when financial problems resulted in "bottom-line" managerial decisions such as wage-freezes and redistributed pay 
scales, employees felt management was violating the spirit of Disneyland and threatening the Disney family. Smith and Eisenberg demonstrated how identifying multiple, changing, and/or competing root-metaphors and their entailments can provide insight into the nature of organizational conflict. This analysis also shed light on the evolution of meanings within the organization as employees and management responded to significant impacts on the organizational reality.

\section{Metaphors and Organizational Transformation}

Sackmann (1989) investigated the possibility that metaphors might be useful tools for communication during the process of organizational transformation. Sackmann describes transformation as going beyond "incremental change" and the "step-wise" improvement or amplification of existing states or conditions (p. 464). Transformation is "revolutionary" rather than "evolutionary"; a fundamental change in the commonly held and collective beliefs, assumptions, feelings, and actions that comprise the culture of an organization. She poses the question: "How can basic assumptions or beliefs be changed that are collectively held and taken-for-granted in an organization?" (pp. 464-65).

Building on the power of metaphor to transmit large amounts of information holistically, to trigger shifts in perception, and to make the abstract concrete with vivid and easily remembered images, she hypothesizes that carefully selected metaphors could be used to "to influence employees' thinking, feelings, and their construction of reality in ways that facilitate organizational transformation," 
initiating the process "by triggering a perceptual shift," in which the choice of metaphors influences "the direction, interpretations of, and feelings about the shift" (p. 469). Two kinds of metaphor are suggested: targeted metaphors that indicate a transformative process toward pre-specified goals and adaptive metaphors that facilitate a process where the "future is unknown and the goals are likely to change" (p. 468). Targeted metaphors, such as "engineering," suggest a very focused approach with clear strategies, tactics, and desired outcomes. Such a metaphor can be useful as long as the relevant environment cooperates by remaining stable and predictable. Since stability of this nature is rare in the case of a human work force, targeted metaphors may not provide the kind of flexibility needed to address the range of activities and presence of unknowns in the process of transformation. Using multiple metaphors might address this need for flexibility.

Sackmann noted that successful organizational transformation could be accomplished using "adaptive" metaphors that can respond to changing conditions and the presence of "unknowns" without becoming obsolete. "Adaptive" metaphors

describe a transformation process whose outcome cannot be specified at the outset. They connote a continuing process of search, taking action, and adjustments toward 'fuzzy' goals which may change during the process" and adaptive metaphors "can accommodate random variation and changing conditions without becoming obsolete. (p. 471)

In Sackmann's case study, the organization expressed verbally and behaviorally the adaptive metaphors of "philosophizing" and "gardening" to indicate a organizational reality incorporating exploration, interaction, collaboration, and joint efforts in an 
ongoing adaptive process. The "philosophizing" metaphor allowed the organization to search out their identity and direction, while the "gardening" metaphor provided ways for implementing and maintaining the new reality negotiated during the "philosophizing" stage of transformation. Adaptive metaphors seem appropriate in situations that require individual "ownership" of changes and an openness to ongoing learning.

Sackmann cautions that while metaphors can be useful, they are not a precise instrument for creating change. Metaphors are still communicated using language, and thus, will not necessarily mean the same thing to different people. Similarly, two different people may use two different metaphors to characterize the same behavior. In testimony to the power of metaphors, one must pay attention to the potential for them to be used unethically, especially when one considers that it is typically people in power who get to impose new metaphors (Lakoff \& Johnson, 1980).

\section{Metaphors, Culture and Issues of Communication}

In chapter one the claim was made that metaphor analysis is an appropriate approach for the study of the meaning structures of cultural systems. Three studies presented in this section develop aspects metaphor and metaphor analysis in the context of culture and communication. The first study is an example of metaphor analysis used to provide insight into Malaysian students shared meanings about their interpersonal relationships and to shed light on problems of cross-cultural communication. The second study examines a pervasive root-metaphor in English 
speakers' discourse about communication--the "conduit metaphor"--and explores the ways in which this "preferred frame" for conceptualizing communication perpetuates particular communication problems and prevents any enduring shift to view communication through a different frame. The third study introduces the concept of "generative" metaphors as "problem-setting frames" and explores the possibility of re-structuring these frames to produce new ways of "seeing as."

\section{Metaphor Analysis and Culture}

Building on the work of Koch and Deetz (1981), Deetz (1984) develops a case for using metaphor analysis as an interpretive approach to reveal meaning structures that are produced and reproduced in the discourse of cultural systems.

Each metaphor is a witness for the system of which it is a part. The appearance of a metaphor in speech at the same time is part of the production of the metaphorical system and reproduces the system that exists. The metaphors in this sense produce their own conditions for being understood" (Deetz, p. 219).

In revealing the systems of metaphors one also gains insight into the coherence of thought and action in a culture and glimpse the way cultural members "structure time and space and orient themselves to things about themselves. . . the patterns of metaphors, the system of similarities and differences they entail, all trace the way a culture thinks" (Deetz p. 219; 220; 221).

Deetz reports on a study in progress that involves the sizeable population of Malaysian students (including Malays, Chinese, and Indians) on the Southern Illinois University Campus. The majority of these students are either required or advised to 
take a basic interpersonal communication course. The difficulty experienced by many of the Malaysian students in the course did not seem explained by the more obvious problems with language, speaking in groups, or different cultural and religious norms about what kinds of personal experiences are appropriate to talk about. The students appeared to experience trouble making sense of parts of the conceptual material which lead to investigating just how Malaysian students (and particularly the Malays) conceptualized interpersonal relationships.

Ten interviews were conducted by a Malaysian student (highly fluent and trained in metaphor analysis) with Malaysian students present in the U.S. for less than two months. The interviews were non-directive in style, eliciting "spontaneous conversation about relationships" (p. 222). Metaphoric linguistic expressions were extracted from these conversations and sorted into metaphoric structures with associated entailments. An informal check with the Malaysian students confirmed the accuracy of the metaphoric structures developed.

North American metaphors for relationships tend to treat a relationship as "an object having a quasi-independent existence" formed through the actions of the individuals. The Malaysian students conceptualization of relationships was considerably different in content and in the implicit ontological claims being made about the relationship and its participants (p. 223). For example, the metaphors did not frame the relationship has having an objective existence independent from the people in relationship. A variety of other differences in the way Malaysian students 
conceptualize relationships emerged from the analysis, creating an opportunity for better understanding between the cultural groups and providing new ways of experiencing familiar concepts. The analysis also suggests that metaphor analysis can be used successfully to investigate the hidden meaning structures that impact both intra- and inter-cultural communication.

The "Conduit" Metaphor and "Frame Conflict"

One of the earliest observations I made about LEC is that they seemed to operate using a different "framework" for conceptualizing issues of communication. Reddy's (1993) research revealed a "conduit" root-metaphor for communication which provides a useful analytic contrast, in addition to illuminating the issue of "frame conflict."

The "conduit" metaphor represents a particular worldview or a "preferred framework for conceptualizing human communication" as if "language transfers" meanings between communicators (e.g. "try to get your thoughts across better," "Her feelings didn't come through to me"; p. 166-67). Reddy describes the conduit metaphor as having a "major" and "minor" framework. In the "major framework" the metaphoric linguistic expressions indicate that

(1) language functions like a conduit, transferring thoughts bodily from one person to another; (2) in writing and speaking, people insert their thoughts or feelings in the words; (3) words accomplish the transfer by containing the thoughts or feelings and conveying them to others; and (4) in listening or reading, people extract the thoughts and feelings once again from the words. (p. 170) 
The "minor" framework suggests three additional categories:

(1) thoughts and feelings are ejected by speaking or writing into an external "idea space"; (2) thoughts and feelings are reified in this external space, so that they exist independent of any need for living human being to think or feel them; (3) these reified thoughts and feelings may, or may not, find their way back into the heads of living humans. (pp. 170-71)

There are at least two important social and psychological implications of the "conduit" metaphor for how human beings approach and enact communication. The first is believing that communication is what Reddy calls "success without effort system," rather than a system that requires considerable energy and effort from participants (p. 186). In other words, if communication is simply putting meaning into the appropriate words and sending it off or if I project meaning objects out into space to be (or not be) collected by others, then successful communication should be relatively effortless.

The second implication of the conduit metaphor is that what little amount of effort it does imply is necessary in communication, is primarily located in the speaker. That is, the speaker must invest some effort in selecting the best word containers for his or her meanings, but once that is achieved, he or she has merely to transfer them to the listener who can then extract the meanings. This viewpoint might also suggest that with practice and experience, a speaker ought to develop considerable skill in this activity. The conduit metaphor, thus, trivializes the role of listening, locating skill (or lack of) in the speaker. 
I see a third implication resulting from the dominance of the conduit metaphor in our understanding and experience of communication: the absence of responsibility. I see the conduit metaphor creating a situation where neither the speaker or listener is required to experience themselves as responsible for creating a successful or effective communication transaction. A speaker, perceiving him or herself as using the appropriate words need not take responsibility for an unsuccessful attempt to communicate. Likewise, a listener can defer responsibility for expending effort toward creating shared meaning to the speaker, who is responsible for putting his or her meaning into the appropriate words.

Unfortunately, it seems awareness of the conduit frame is not enough to exorcise it from our discourse about discourse. According to Reddy, while we may be able to think in terms of another frame for periods of time, the conduit metaphor will likely persist until or unless rigorous renovations of the English language cease the reproduction of the framework of our everyday speaking:

The logic of the [conduit] framework runs like threads in many directions through the syntactic and semantic fabric of our speech habits. Merely becoming cognizant of this in no way alters the situation. Nor does it appear that one can adopt a new framework and develop it while ignoring the cloth of the language. For everywhere one runs into the old threads, and each one pushes conversation and thought back a little way toward the established pattern. . . I do not claim that we cannot think momentarily in terms of another model of the communication process. I argue rather, that that thinking will remain brief, isolated and fragmentary in the face of an entrenched system of opposing attitudes and beliefs. (Reddy, 1993, p. 176-77)

The phenomenon of "frame conflict" is by no means unique to the domain of 
communication. The underlying principles suggested here have implications for the entirety of this study where the metaphors being analyzed are situated in an alternative framework for viewing the world.

\section{Generative Metaphor, Frame Awareness, Frame Restructuring}

Schon (1993) explores what he calls generative metaphor: the process of "seeing as" in which "frames or perspectives" of one domain of experience are imposed on another "by which we gain new perspectives on the world" (p. 138). Using examples from the domains of product development and social policy, Schon demonstrates the way in which generative metaphors act as problem-setting frames, generating a particular way of seeing a problem that also sets the directions of problem solving. Clues to generative metaphor are present in the language used to tell stories about a problem or situation. He points out that since we do think metaphorically about problems we need to develop "frame awareness"--a critical awareness of generative metaphors. Frame awareness may also increase our attention to and experience of conflicting frames, thus creating the possibility for "frame restructuring."

Schon points out that "frame conflict" tends to be unresolvable by factual appeals became the different frames actually focus attention on different facts. Schon poses that responding to frame conflict by "constructing new problem setting stories that attempt to integrate conflicting frames"--the process of frame restructuring--is a process very similar to the making of generative metaphor ( $\mathrm{p}$. 
152). In this process, the old frames are not mapped onto one another; but rather, restructured to coordinate with one another. "The cognitive work of restructuring draws upon the richness of features and relations which are to be found in the concrete situation" (p. 158) from which individuals begin to construct new stories and descriptions about the issue or problem being addressed. Schon notes that research needs to address the particular kind of stance required that will allow people to "recognize descriptions as descriptions rather than as reality" (p. 160).

Schon's work does not address the sort of difficulties that Reddy (1993) points out regarding the relative embeddedness of certain frames in the larger context of the our conventional language. He does point out, however, many of the potentially necessary components in creating successful shifts.

Issues of culture, communication and the problems and possibilities of managing differing metaphoric frames are particularly relevant in this study of a cultural context whose metaphors are situated within both the process of producing a paradigm-shift and the alternate view of reality that is the product of that shift.

\section{Descriptive Framework}

Metaphor analysis may proceed in a number of ways, with or without further framing. This study was informed and contextualized by a number of sensitizing concepts that organized my ideas, observations, and analysis during the process of carrying out this study (Bulmer, 1979). The ethnography of communication sensitized me to the issues of the cultural embeddedness of language and organized 
my observations of LEC as representing a "speech community" with a distinct "way of speaking." The concepts of empowerment, transformation and community are presented together as a body of thought that guided my observations regarding the experiences of Landmark's participants (e.g. the consequentiality of participation). The social construction of reality could not be ignored in this study, both in terms of the processes of creating and maintaining any social reality, and because LEC incorporates social constructivist premises into the system of meanings and practices that comprise their worldview. The works presented here are offered as a "descriptive framework," a disclosure of those concepts that I, the research instrument, take into this endeavor:

the specification of a descriptive framework permits the systematic detection and, perhaps, correction, of researcher bias and partiality; if there is to be to be partiality, and inevitably there will be, let it be deliberate and therefore detectable, rather than unwitting and therefore insidious and enigmatic. (Philipsen, 1977, p. 47)

\section{Ethnography of Communication: Speech Community and Ways of Speaking}

Metaphor, according to Lakoff and Johnson (1980), is both linguistic and conceptual, and our access to the conceptual aspects is through linguistic expressions. They repeatedly draw attention to fact that our understanding and use of metaphor is culturally situated and constituted. The linguistic expressions and metaphoric mappings that are the focus of this study are approached with the assumption that "speaking is, fundamentally, speaking culturally" (Philipsen, 1992, p. 124). As a cultural context, Landmark and its participants share a speech code: 
"historically transmitted, socially constructed symbols and meanings, premises and rules, pertaining to communicative conduct" (Philipsen, 1992, p. 124). For example, LEC is a primarily oral culture in which participants highly value both speaking and listening as creative acts. This aspect of LEC's speech code alone has implications for the rules, patterns, topics and meanings of communication in this culture (Philipsen, 1992).

Consider for example, the vocabulary and value for achieving a sense psychological completion in a conversation shared by LEC participants:

... its, very effective in, relating an idea when you can use a certain language, like "let's get complete" on something . . . Somebody [in LEC] knows exactly what you mean--"I'm not complete on that." And Landmark's very big on getting complete, which is really cool. Cause most people, don't. . . . there are a lot of questions left hanging in the air at end of a conversation, many times, and people aren't sure, to bring those questions up ... if the other person is already disengaged and starting to walk away. . . I think Landmark people are very quick to say: "I'm really not complete on, on this," or "Let's get complete on that." And the other person, instead of being put out, completely understands, and you'll talk it out until you're complete. (Simon, 1367-85)

Simon suggests that he has observed a contrast between the communicative values and practices as experienced through LEC, and those of the larger culture within which Landmark is embedded.

Thus, the context of culture was sensitized in this study by the concepts of the ethnography of communication. The ethnography of communication was called into being by Dell Hymes in 1962 with the publication of his essay, The Ethnography of Speaking. Hymes advocated a synthesis of linguistics (and specifically, 
sociolinguistics), anthropology, literary and folklore studies, and natural-language philosophy, to name a few of the relevant disciplines (Saville-Troike, 1982; Bauman \& Sherzer, 1974), in a framework he outlined to "guide and motivate inquiry into speech behavior" (Stewart \& Philipsen, 1984). Hymes maintained that language is situated in a context of culture, and cannot be understood for what it is separate from how and why it is used in social life:

As to basis: one cannot take linguistic form, a given code, or even speech itself, as a limiting frame of reference. One must take as context a community, or network of persons, investigating its communicative activities as a whole, so that any use of channel and code takes its place as part of the resources upon which the members draw. . . The ethnographer is likely to look at communication from the standpoint and interests of a community itself, and to see its members as sources of shared knowledge and insight. I believe that the only worthwhile future for the science of man lies in the realization of such an approach. (Hymes, 1974, p. 4, 8)

The goal of ethnographers of communication, then, is "to discover, describe, and interpret patterns of speaking in particular speech communities" (Braithwaite, 1991, pp. 145-146).

With the above goal in mind, the central focus of ethnography of communication is the "speech community" defined by Hymes (1972) as: "a community sharing rules for the conduct and interpretation of speech, and rules for the interpretation of at least one linguistic variety" (p. 54). Braithwaite (1991) defines an approach for identifying a speech community in which the observer defines and describes the degree to which: 
(1) a group of speakers share aspects of linguistic variation (i.e., covariation is present between language and social context), (2) a group of speakers share communication rules for speaking (i.e., the use of speech judged as appropriate by native speakers), and (3) shared meaning is present among a group of speakers (i.e., members successfully render coherent and intelligible speech in the community). (p. 146)

Thus, Braithwaite's approach requires that the analyst go beyond the simple identification of the existence of a speech community to making a case for varying degrees of community.

Saville-Troike (1982) notes, and linguists generally agree, that a speech community cannot necessarily be equated with those who share a common language, as speaking the language is sufficient for some degree of participation, but membership in the community is comprised of more than language knowledge and skills. Saville-Troike suggests that ethnographers begin with an "extralinguistically" defined social community since patterns of language use and interpretation and other sociolinguistic elements are product of the ethnographic exploration. This minimizes the problem of circularity arising from using the same criteria to determine and describe a speech community. Social science definitions of community all include some notion of shared knowledge, meanings, behaviors, possessions, interests, etc. One could begin there and then investigate the proposed community's communicative "repertoire in terms of the socially defined community" (p. 19).

Hymes outlined the construct of "ways of speaking," as indicating a distinctive way of "doing" communication and distinctive way of life, which together create a 
unique perspective for studying social life. This concept has been further developed and modified to include three key constructs: "spoken life," "ways of speaking," and "cultural communication" (Stewart \& Philipsen, 1984, p. 209). Spoken life refers to the facet of life in which spoken forms of interaction are the primary focus. A way of speaking is an interpretive and explanatory term denoting culture specific meanings of identity intersecting with the "most audible terms, tropes, modes and contexts for communication" (p.209). Cultural communication is concerned with the "communicative activity which performs the function of defining, affirming, and negotiating a shared sense of meaning" among a community of speakers (p. 209).

In summary, the guiding hypothesis for an ethnographer of communication is that language resources, structure and use are cross-culturally variable:

Communities differ as to what linguistic resources are available to their members, how linguistic resources are patterned in use, the functions that are served by language, and the valuation of language as an instrument of action in social life. Because they are variable, these resources, patterns, functions, and values must be discovered in each case. (Philipsen, 1977, p. 44).

\section{Empowerment, Transformation \& Community}

The concepts of empowerment, transformation, and community are related to this study via the observations made about participation in Landmark in chapter one. Namely, that the "transformative" experience of participation in LEC's courses seems to provide participants with both a sense of personal empowerment and collective identity that I have labeled community. Consider some of the comments 
participants themselves shared regarding their experience of community and empowerment:

Transformation in all areas, in communication ... going from a level of just me, the world is about me, just being so concerned with yourself and your life; versus really going to a point of being able to step outside that and function outside of that realm--I see that as transformation in communication. Because it provides something more in what can be done and what can be possible between people and what we can accomplish on the earth ....

[T]ransformation as far as just in my own life? I guess it would go back to having choices about things and possibilities, new possibilities and being able to choose different things then I chose before. . . Just being aware [of] what words I choose, the power of the words I choose. . . . Definite big huge transformation in what reality is. . how much power you have in creating reality ... what's reality for someone else and what reality are they living in. (Jenna, 942-1007)

the biggest impact it had on my life was just the connection with other human beings. . . Just the unbelievable humanity of people, paying attention to other people and really listening to what they said. . . How many people did that, 150 people? And just feeling connected with all of them all at once and it was . . almost overwhelming it was so cool. You know, and that was, I've never had anything like that. . . I have close friends, sure. But even that--that big group of people and everybody just being positive and saying "I will listen to you" you know, "say what you want." That was so unbelievable. (Mora, 146163)

... that's one of the great things that Landmark gives you . . you have a whole lot more ... love and respect, people being people, you're very understanding of people and kind of that unconditionality, cause that's the way humans are. . . one of the great things is, you thought that you were alone with all these weird feelings before the Forum and then you realized, one of the great breakthroughs in the Forum, is that you realize that we're all that way. And . . . all of a sudden, you become this community. . . (Simon 367-385)

These excerpts suggest that a potential outcome of participation in LEC's courses is a shift in an individual's experience of their relationship to other people that might indicate the possibility of "community." An awareness of the power of communication seems to be an important component in these experiences (especially 
notable in excerpts one and two) as does the development of empathy (excerpt \#3). Also suggested in these excerpts is that this experience of connection or community may be associated with feelings of empowerment. The research presented in this section explores the proposed relationship between empowerment, community and transformation

Rappaport (1984) provides a description that captures some of the complexity of empowerment that makes it difficult to define and study:

Empowerment is viewed as a process: the mechanism by which people, organizations, and communities gain mastery over their lives. However, the content of the process is of infinite variety and as the process plays itself out among different people and settings the end products will be variable and even inconsistent with one another. . . For some people the mechanism of empowerment may lead to a sense of control; for others it may lead to actual control, the practical power to effect their own lives. . . Empowerment is easy to define in its absence: powerlessness, real or imagined; learned helplessness; alienation, loss of a sense of control over one's life. It is more difficult to define positively because it takes on a different form in different people and contexts. (p. 3)

Empowerment, then, is both process and product, knowable only in the observable forms it takes, and yet, those forms may vary divergently:

"Understanding that $\mathrm{H}_{2} \mathrm{O}$ can be in liquid, gas or solid form is like the realization that empowerment for a poor, uneducated black woman can look very different than for a middle class college student or thirty-nine year old businessman" (p. 3). In general empowerment can be defined as "enhancing the possibilities for people to control their own lives" (Rappaport, 1981, p. 3). 
Kieffer (1984) concurs with Rappaport, commenting that while the idea of empowerment has become increasingly popular since the 1960s, and is an appealing concept for both theory and practice, its "applicability has been limited by continuing conceptual ambiguity" (p. 9). In order to gain a better understanding of what empowerment is Kieffer conducted in-depth interviews with 15 individuals who were characterized by 1) self-acknowledgment of personal transformation, 2) recognizable transition into proactive and multi-issue engagement, and 3 ) evidence of continuing commitment to involvement in local political processes or grassroots leadership roles. Kieffer asks the questions: How do individuals manage to move beyond their experience of powerlessness and oppression? In what ways do the shared experiences of these individuals provide insight into the idea of empowerment? Kieffer found that two themes occurred pervasively throughout people's experience of empowerment. The first he described as

a function of a continuing internal 'constructive dialogue' or the maintenance of the creative force of internal contradiction. . . In the struggle towards empowerment, conflict and growth are inextricably intertwined. It is essential that individuals continue to experience conflict to sustain their emergence. It is also essential that there be constructive channels and supportive resources for resolution of these continuing internal confrontations. (p. 25)

The second theme is that of the "dynamic of praxis": a cyclical process experience and reflection that evokes new understandings and hence, new and more effective actions (p. 16). This cycle of action and reflection is integral to learning and accumulating the experience and skills at the heart of empowerment. Kieffer 
suggests that "empowerment is a transforming process constructed through action" (p. 27). Empowerment can also be conceptualized as enduring body of commitments and capabilities Kieffer calls "participatory competence." "Participatory competence" is composed of three interrelated dimensions: (1) developing a more positive self-concept and sense of self-competence; (2) constructing critical and analytic skills for inquiring into the surrounding conditions of existence; (3) the cultivation of individual and collective resources for being in action (e.g. social, political, etc.).

Katz (1984) claims that because the process of empowering others involves dealing with the "generation and distribution of power in the form of access to and control over resources" (p. 204), empowerment tends to exist within what he calls a "scarcity paradigm." The "scarcity paradigm" tends to characterize Western thinking about the existence and distribution of resources. In assuming valued resources are scarce, individuals and communities must compete for them, accumulate them, resist sharing--indeed, it is often their supposed scarcity that makes them valuable. Katz further suggests that there is an alternative paradigm that can free human resources from the limiting perspective of scarcity: a "synergistic paradigm." Katz develops a model of "synergistic communities":

Synergistic community is a perspective for understanding the functioning of synergy within a community as well as a guideline for increasing synergy. In the context of a "synergistic community,' empowerment expands in an exponential fashion. Communities empower and become empowered. A synergistic community can be defined as one in which: 
1. A valued resource is renewable, expandable, and accessible.

2. Mechanisms and attitudes exist which guarantee that the resource is shared equitably among community members.

3. What is good for one is good for all.

4. The whole is greater than the sum of the parts. (p. 209)

Katz says that the field data indicate that a synergistic community is established by

"rituals of transformation" in which participants experience a transformation of consciousness. This is not necessarily a major, intense or radical alteration of consciousness. Nor is the ritual necessarily a dramatic or clearly demarcated event. . . This transformation seems to bring on a (new) way of experiencing self as embedded in and expressive of community. . . To establish the empowering environment which is found in a synergistic community requires a radical paradigm shift--a major shift in the way persons experience meaning and process and interpret data. (p. 208, 222)

"Education as transformation" is one method to create "rituals of transformation." The key to such a model of education is transforming consciousness such that a new experience of reality "in which boundaries of the self become more permeable" results in a sense of connectedness (p. 223). Katz also suggests that the transformation of consciousness in a recurring process of transition, rather than one of stages reached, barriers crossed, or definite outcomes.

Michael Lerner's (1986) work focuses on the concept of "surplus powerlessness," an psychological experience which, until addressed, precludes the possibility of any enduring experience of empowerment. Where powerlessness is the recognition and acknowledgement of social conditions that makes some individuals and groups more equal than others (i.e. class differences, gender differences, etc.), surplus-powerlessness is the perception that an individual is completely without 
power. Lerner claims that systematically maintained environmental conditions-reified structures of the dominant paradigms regarding success and failure, work life, etc.--undermine the human actualization we all seek, creating fragmentation and isolation, and eventually transforming powerlessness into surplus powerlessness. Lerner argues that the undermining of human actualization begins when we enter into and inherit a world constituted in language:

The language of "reality," the language that constitutes the world for us, simultaneously proclaims to us that the world as constituted is justly constituted. The very act of entering the linguistic system means entering into a world of meanings in which the fundamental fairness of how things are set up is assumed and asserted. (p. 147).

When people begin to act on their internalized notion of surplus powerlessness-when they accept that fragmentation and isolation are part of the way the world is-there is no longer room for the possibility of change, and thus, the actions that would facilitate change. Lerner claims that anything countering isolation would be the starting point in preventing and altering the condition of surplus powerlessness. Not surprisingly then, part of his answer to surplus powerlessness is creating community. He calls for a "psychology of compassion" and "compassion groups" as integral to undermining surplus powerlessness and creating movement toward personal and social action:

I believe that the first step towards building a mass psychology of compassion can best take place in a context in which people come together to tell each other their stories. While the frame of these groups may differ in terms of the initial reason why they come together, their shared purpose must be to create public spaces within which we listen attentively and 
caringly to others and let them hear us. Groups that do this can play a critical role as a first step for many people (p.339-340)

In order for such groups to be successful they would need to unconceal and disarm the invisible presence of reified conditions that contribute to the experience of fragmentation and isolation and develop empowering structures to replace it. The revealing of shared experiences begins the process of lessening isolation, as people begin to see that what they perceived as their own personal hang-ups are actually shared and collective experiences. Lerner describes how compassion groups can become "communities of compassion," where one

is no longer identifying oneself as a problem case by joining the group; but, rather, one is participating in a social movement, a cause, a common project, a newly emerging community. The meaning of the experience is totally different. . If hundreds of thousands of people were participating in these groups and talking about what they were learning about themselves, that fact would make people believe that something could be different in the rest of their lives. ( $p$. 341)

What Lerner suggests is possible out of such groups is various forms of "transformation" leading to the experience of self-as-powerful, and thus, personal action in a variety of forms and contexts.

\section{Social Construction of Reality}

The body of thought labeled the "social construction of reality" covers a range of ideas that share the basic premise that human meanings, understandings, and realities arise, persist, and can be altered through our communicative practices and processes. (Littlejohn, 1992). Berger and Luckmann's (1966) well known treatise, 
The Social Construction of Reality, systematically develops a comprehensive explication of the processes by which human beings produce, participate in and are impacted by constructed realities.

Language and social interaction, or conversation (which includes intra-personal communication), are the primary means by which subjective realities become "objectivated" and maintained through habitualized, institutionalized, and sedimented meanings and practices. "An understanding of language is thus, essential for any understanding of the reality of everyday life" (Berger \& Luckmann, 1966, p. 37). An institutionalized world is experienced as objective reality--reified.

Reification implies humans become forgetful of their own "authorship in the world" as the "dialectic" between human, "producer, and product is lost" (p. 85).

Berger and Luckmann also describe the processes by which social realities, and thus, objective realities, can be transformed or altered to varying degrees through processes of "resocialization" that must include a variety of "social and conceptual conditions":

The most important social condition is the availability of an effective plausability structure, that is, a social base serving as the 'laboratory' of transformation. This plausability structure will be mediated to the individual by means of significant others with whom he [or she] must develop strong affective ties. . . Alternation is predicated on a reorganization of "the conversational apparatus. The partners in significant conversation change. And in conversation with new significant others subjective reality is transformed. It is maintained by continuing conversation with them, or within the community they represent. (p. 157;159) 
The most important conceptual requirement for an alternation of reality is a readily available "legitimating apparatus" that will account for the sequence of transformation and provide the means by which the "old reality," including the social relationships and networks associated with the "old reality," can be reinterpreted within the new reality. In describing variations of and conditions leading to successful and unsuccessful socialization, Berger and Luckmann note that an individual may internalize a new reality, but rather than it being his or her reality, "it is a reality to be used" by him or her for specific purposes.

A society in which discrepant worlds are generally available on a market basis entails specific constellations of subjective and objective reality and identity. There will be an increasingly general consciousness of the relativity of all worlds, including one's own, which is now subjectively apprehended as "a world," rather than "the world." (p. 172-73).

This brief discussion barely scratches the surface of the implications for social construction of reality, and may not have introduced all of those ideas most relevant to this study. The social construction of reality contextualizes this study in at least two fundamental ways. First, much of LEC's educational material and methods actually incorporate social constructionist assumptions, making the social construction of reality part of their worldview and resultant practices. For example, participants in LEC seem to develop a heightened awareness of how their intrapersonal conversations shape and direct their subjective experiences and social actions: 
So when they [in the Forum] said that there were these conversations that you have with yourself, it just was like, hit it! . . because I used to catch myself that I would make these, what I called assumptions, not knowing they were really the conversations that I was having to keep myself, either justified or righteous or you know, and so its really freeing to know that, that is a conversation that I'm having with myself, you know, and that it really is destructive. It keeps me from having what I really want. Its not beneficial to me. So, when I say conversation, its this internal voice, that's almost, not my friend, you know. It keeps me from really, having the best of what I could have. (Leigh, 975-990)

Something else that was said in the Forum is that "life is a conversation." And when you can distinguish the conversations that you have, the automatic ones that come from, lets say your past, or comes from something that happened, you know, yesterday or the day before or when you were three years old--when you can distinguish that they were started then, as conversations, you don't have to automatically be run by them. . . . You then have the power to choose another conversation to have about the matter. (Ivan, lines 459-71)

Notice that becoming aware of subjective conversations includes the idea that past "conversations" continue to shape reality in the present. Helping people create an awareness of the crucial role of conversation in the creation and maintenance of subjective and objective reality is a central focus in LEC trainings.

The second way that the literature on social construction of reality applies is that the paradigm-shift and social reality associated with LEC appears to be created as an "alternation" of reality. Indeed, the processes Berger and Luckmann describe as contributing to creating such alternations are resonant with elements of Landmark's course design. For example, the intense and intensive experience of being in a course like the Forum, the experience of strong affective ties with other participants and the development of a very particular vocabulary and way of 
speaking that, if one chooses to continue association with LEC, necessitates at least some form of restructuring of one's conversational apparatus.

\section{Chapter Summary}

This chapter introduced the theoretical framework for this study, beginning with an explanation of Lakoff and Johnson's (1980) and Lakoff's (1993) contemporary theory of metaphor including: metaphors as mappings of experiential conceptual domains; and metaphoric linguistic expressions as generated from those mappings; and conventional, structural, orientational and ontological categories of metaphor. The literature review covered the use of metaphor analysis in organizational settings to investigate shared realities, conflict, and the process of organizational transformation. Studies using metaphor analysis to illuminate the influence of culturally embedded meaning structures on communication, as well as processes to generate new meaning structures, were also presented. Finally, the descriptive framework contextualizing metaphor analysis in this study was presented including: Ethnography of communication, empowerment, community, transformation and the social construction of reality. Chapter three will describe this study's research design, data collection methods, issues of reliability and validity and analytic procedures. 


\section{CHAPTER THREE--RESEARCH METHODS}

\section{Qualitative Approach}

The purpose of this study was to describe and understand the system of metaphors that shape and represent the patterns of thinking, being and acting in the "culture of participation" or community associated with Landmark Education Corporation. A qualitative approach was selected as most appropriate toward fulfilling this purpose. The unique strengths of a qualitative approach are best suited for research that is "exploratory or descriptive, that assumes the value of the context and setting, and that searches for a deeper understanding of the participants' lived experiences of the phenomenon" (Marshall \& Rossman, 1995, p. 39).

Participants' discourse and course notes, as well as organizational artifacts and the researcher's own lived experience as a participant in this community all became the "texts" for the explication and interpretation of LEC's metaphoric system:

Culture as it is to be understood is manifest in various empirical objects and activities including artifacts, forms of movement and speaking. ... An interpretive analysis of culture investigates these interconnections, revealing the system that supports the sense of these objects and activities. (Deetz, 1984, pp. 216-17)

As such, research goals were consistent with those of interpretive, hermeneutical inquiry (Deetz, 1984). Generally speaking, the goals of hermeneutic inquiry are to develop explanations and understanding through the interpretation of human artifacts and actions (Stewart \& Philipsen, 1984). The hermeneutic perspective also 
allowed for the self-reflexivity required of me as both researcher and participant in the community being researched.

\section{Data Collection}

Decisions regarding data collection procedures were contingent on negotiated access with LEC. Data collection methods included in-depth interviews, participantobservation, and acquisition of cultural documents/artifacts into the research log.

\section{$\underline{\text { Access }}$}

Submission of a detailed proposal to LEC (see Appendix A) resulted in the negotiation of conditional and limited access with the Chief Executive Officer by telephone. Appendix B documents, in detail, the negotiated conditions of access. The primary impact of limited access was a greater reliance on already available cultural artifacts and constraints on interviewee selection to individuals within my "personal network of relationships" (see Appendix B).

\section{Interviews}

A series of five "intensive" interviews or "guided conversations" (Lofland \& Lofland, 1995, p. 85) were conducted in order to discover participants' lived experiences of LEC's courses and concepts. McCracken (1988) notes that "less is more" when selecting respondents for intensive interviewing, since qualitative research is "much more intensive than extensive in its objectives" (p. 17). In addition to becoming the "texts" from which metaphoric expressions could be abstracted and 
analyzed, the interviews revealed relationships among abstracted meaning structures through detailed explanations, stories, and examples.

\section{Participant Selection}

The conditions of the agreement with LEC required the selection of interviewees from my personal network of associations as a participant in this community, constraining my choices to a certain amount of convenience. In addition to limitations of access, recruitment of participants was informed by two assumptions offered by Honigmann (1970): (1) "a common culture is reflected in practically every person, event, and artifact belonging to a common system," and that (2) "questions asked in the research can frequently be answered through samples selected by nonprobability methods" (p. 271). Following from these assumptions, this study used both convenience and judgment sampling (Honigmann, 1970). In general, judgment sampling allows the researcher to select participants whose experience, qualities and knowledge inform the goals and design of the research (Honigmann, 1970; Babbie, 1992).

Interviewees' participation with LEC varied from just the Forum (the minimum criteria for inclusion in the sample) to the completion six or more courses and some volunteer assisting with the organization. Another factor considered was the participant's level of formal education as that might influence the degree to which an individual adopted and/or integrated LEC's material (see Appendix C for data tables 
that present descriptive break downs of the interview data for both the pilot and thesis projects).

\section{Interview Process}

Interviews were scheduled at a time and place convenient to the participant. Care was taken to create a setting that was appropriate given our personal relationships, while being conducive to the goals of intensive interviewing. All interviewees had been notified in advance of my desire to audio record the interviews and all consented to this procedure. In addition to allowing me to attend fully to the interviewee and their responses, tape-recording insured accurate texts using native terms and linguistic forms, captured variations in the interview guide and provided an opportunity to monitor my interviewing skills (Ely et al, 1991; Lofland \& Lofland, 1995).

The interview process began by going over the informed consent form (see Appendix D) together before asking them to sign it. Special care was taken to be certain the participant understood LEC's position regarding the research being undertaken, as well as the procedures I would employ to insure confidentiality (pseudonyms, exclusion of identifying information). Any questions the participant had were answered and they were offered a copy of the consent form. Before asking the first question I reminded the participant that in my role as researcher I would endeavor to hear their meanings, rather than assume I understood, and thus, would likely be asking them to elaborate on many things that we would normally take for 
granted between us. Interviews ranged in length from 70 to 170 minutes. Extensive notes were not taken during the interview, however, a post-interview research log entry was done as soon after the interview as possible. The log entry was an opportunity to capture observations, questions, concerns, and methodological or theoretical issues.

\section{Interview Guide}

The interview guide (see Appendix E) was adapted from the materials tested in the pilot study. Questions were worded so as not to bias a particular metaphor (e.g. not asking "How have you used LEC material in your life" which might bias ontological metaphors or suggest a tool metaphor). Due to the potentially variable nature of the interviewees' background and experiences with LEC, the interview guide was designed to be used as a flexible format, rather than a tightly structured schedule of questions:

an [interview] guide is not a tightly structured set of questions to be asked verbatim as written, accompanied by an associated range of preworded likely answers. Rather, it is a list of things to be sure to ask about when talking to the person being interviewed. . . . You want interviewees to speak freely in their own terms about a set of concerns you bring to the interaction, plus whatever else they might introduce. (Lofland \& Lofland, 1995, p. 85, emphasis in original)

Thus, the interview guide became something of checklist to make sure intended topics were covered (Lofland \& Lofland, 1995). This flexible format provided a framework within which the participant could exert influence on the direction the interview took and address topics of importance not specifically included in the 
outlined interview guide (Ely et al, 1991; Patton, 1996). The notion of a "guided conversation" also more readily accommodated the dynamics of the personal relationship shared with each participant.

\section{Participant/Researcher Relationship}

McCracken (1988) notes that qualitative research typically involves a more demanding form of relationship between the researcher and the respondents. In this study, the respondents and I shared personal relationships (e.g. had engaged in some social activities external to LEC) contextualized by our mutual participation in LEC. Our shared membership in LEC's community was the most salient influence on the interviews.

One of the most interesting observations I made regarding the interviews was that my shared membership with the respondents brought LEC derived values, norms, and practices to bear on the interview context. One observation had to do with the experiential domain of "sharing," a highly valued and continuously reinforced LEC practice, being superimposed on the domain of interviewing (see chapter four pp. 115-120 for a detailed explanation of "sharing" as used in the LEC context). "Sharing yourself" in LEC is distinct from "talking about" or "explaining." Review of interview transcripts and log entries suggest that both I and the interviewees interpreted the interviews as opportunities to "share" their experiences with me. Two interviewees explicitly referred to their interview responses as "sharing" (Leigh, 1215-27; Simon,) and I frequently used the word "sharing" to refer 
to participant responses during the interview. Several participants indicated that the potential outcomes of "sharing" (creating insights, bringing their "transformation" present, etc.) were realized for them during the interview (Leigh, lines 1215-27; Mora, 1164-66; Ivan, 2813-2837). McCracken (1988) concurs that intensive interviewing offers potential benefits for respondents, as well as researchers.

During one interview, a woman who had just completed the Forum seemed to repeatedly diverge into tangential personal stories or insights. During post-interview logging I speculated that these tangents were a product of her "sharing" what for her were very recent "transformative" experiences. My own difficulty in managing the conversation I saw as influenced by the norms of "sharing" which created some tension for me between my roles as interviewer and participant. As interviewer I wanted to steer her back to the interview guide; as cultural member I wanted to listen generously and without "in-order-to's," that is, not listening as a means to an end.

This transference of LEC values and communicative practices into the interview context was overall, more advantage than disadvantage. LEC participants tend to come to value conversation as a creative act, which has implications for the perceived roles of listening and speaking. The LEC values of openness and authenticity, the context of safeness, acceptance, and connection that comes with shared membership, and the personal aspect of our relationships all contributed to participants' willingness to share their experiences and opinions freely. 


\section{Participant Observation}

The primary goals for participant observation were: (1) to be exposed to and participate in spontaneous and natural conversations that might provide insight about how metaphors were or were not realized in speech and behavior; (2) to participate in the community in a unfamiliar setting that might help manufacture distance by forcing me to experience LEC and myself in new ways (McCracken, 1988).

Participant observation took the form of a six month "assisting agreement"1 to work in an LEC office or "Center" for $31 / 2$ hours per week performing data entry and answering phones. Because of the access agreement negotiated with LEC, my role while at the Center was almost purely "assistant" rather than "researcher." The Center is a bustling and, much of the time, understaffed office. The working conditions were often tinged with urgency and there was always ample work to be done. Access conditions made it inappropriate for me to ask research-specific questions while at the Center. Office conditions made it nearly impossible to jot notes most of the time, however, and the office norms did not accommodate frequent runs to the rest room to record notes, as seasoned researchers report they

\footnotetext{
1"Assisting agreement" is the term LEC uses to denote a formal agreement to assist in a particular capacity for a specified amount of time. The agreement is formalized as a signed document.
} 
often do. Detailed descriptions of assisting sessions were logged as soon as possible afterwards.

\section{The Research Log}

The research log consisted of accumulated entries of various kinds including: notes taken by participants during LEC courses; organizational documents and artifacts; "field" notes; interview summaries; records of conversations not occurring during interviews or observations, but pertinent to the study; personal entries not specifically attached to interviews or observations containing reflections, musings, concerns; secondary data from the pilot study; and analytic memos. Ely et al (1991) identify the $\log$ as an integral part of the ongoing process of data collection and analysis:

As much as possible, our logs are chronological records of what we learn and our insights about how we learn it. ... The log contains the data upon which the analysis is begun and carried forward. It is the home for the substance that we use to tease our meanings and reflect upon them as they evolve. The log is the place where each qualitative researcher faces the self as instrument through personal dialogue about moments of victory and disheartenment, hunches, feelings, insights, assumptions, biases, and ongoing ideas about method. (p. 69)

Ely et al (1991) also emphasize that the $\log$ is the data; only that which is captured in the $\log$ is available for analysis and inclusion in the research endeavor.

\section{Course Notes}

LEC does not produce or distribute any form of recorded course materials such as manuals, books, or tapes. In addition, access restrictions prevented me from interviewing informants employed by LEC or viewing the materials course leaders 
train from. Thus, my sources for "texts" more central to LEC as the source of the metaphoric system were limited. Participant notes taken during LEC courses seemed the best alternative data source to address research needs. While such notes are subject to the problems inherent in note-taking (perceptual differences, listening skills and habits, etc.), it was reasonable to consider that notes would represent cultural meanings closer to those actually disseminated by the organization. In other words, during a course, people listen to "capture" what is being "taught," whereas discourse about experiences would tend to represent internalized, negotiated and thus more variable meanings. Course notes, in combination with other organizational artifacts, provided a sort of model of LEC's most salient metaphors which could be then compared, contrasted and woven into a more complete picture of LEC's metaphoric system.

Course notes used in this study were my own and those borrowed from an informant volunteering to share this information with me. Having the informant's notes served as a form of triangulation (Lincoln \& Guba, 1985) by providing a second source for this information. LEC courses represented in this sample are as follows: the Advanced Course (AC); the Self-Expression and Leadership Program (SELP); the Forum in Action seminar (FIA); the Breakdown seminar (Brkdwn); the Communication: Access to Power course (CAP); and the Relationship seminar (Relshp). All course notes, with the exception of one set, were taken prior to the conception of this study. 


\section{$\underline{\text { Documents/Artifacts }}$}

Promotional brochures, program descriptions, and LEC-produced organizational documents were collected to provide another body of texts from which metaphors could be abstracted. These were used in combination with course notes to contribute to the construction of the metaphoric system and to enhance understanding of LEC's worldview (Koch \& Deetz, 1981).

\section{Reliability and Validity}

All who propose to engage in scientific inquiry must consider issues of reliability and validity and include in their research plan, methods and techniques for increasing the trustworthiness and demonstrating the credibility of their findings (Lincoln \& Guba, 1985; LeCompte \& Goetz, 1982). While research in the qualitative/naturalistic paradigms differs from that in the quantitative/positivistic paradigm in their goals, methods, and underlying assumptions, they have as much need for reliability and validity as any other form of scientific inquiry (Kirk and Miller, 1986). In all forms of research, reliability is concerned with replicability of research findings and validity with the accuracy of findings:

Reliability is the degree to which the finding is independent of accidental circumstances of the research, and validity is the degree to which the finding is interpreted in a correct way. ... Objectivity is the simultaneous realization of as much reliability and validity as possible. (Kirk \& Miller, 1986, p. 20) 


\section{Reliability}

Establishing reliability, while challenging in any form of research, poses special challenges for those conducting qualitative inquiry due to the nature of the data and the research processes involved in collecting and analyzing qualitative data:

Because of factors such as the uniqueness or complexity of phenomena and the individualistic and personalistic nature of the ethnographic [this term used to refer to a variety of qualitative designs] process, ethnographic research may approach rather attain external reliability. (LeCompte \& Goetz, 1982, p. 37)

The most significant challenge to reliability in this study was my "researcher status position" within the community of LEC. My unique position in this community posed a considerable challenge to the reliability of this study. Extensive participation in the research context means that to a certain extent, LEC's ways of thinking and speaking, their values, norms, and practices, have become my own or at least are very familiar to me. This has implications for whether or not an independent researcher without this degree of participation would come to the same analytic conclusions as I. Physical access to information as well as the degree of trust afforded me were additional factors of my social position that complicated reliability. Replication would require the researcher either be or become part of this community by participating in LEC's courses in order to develop a corresponding position (LeCompte \& Goetz, 1982). Without minimal membership (i.e. completing the Forum) a researcher might have limited access to informants (unless he or she knew people in the community) and little access to other than promotional material. 
A researcher with a more limited social position could still produce legitimate results, but not achieve replicability. However, the choice was made to make use of my social role in order to provide insight into a virtually unstudied culture. Lofland and Lofland (1995) acknowledge the importance of making informed research choices: "We urge only that your decision be consciously made and that you clearly articulate to yourself the basis or bases for making it" (p. 27).

Conscientious, thorough, explicit, confirmable and ongoing explication and documentation is the recommended method for addressing nearly all issues of reliability (Kirk \& Miller, 1986; LeCompte \& Goetz, 1982). In this study, the research log was used extensively for reflecting upon and recording my role as the research instrument (biases, assumptions, reactions, inferences, etc.); for explicating theoretical premises and defining concepts; for careful description of data collection methods, sources, and contexts; and for detailed documentation of analytic procedures and findings.

\section{Validity}

Two questions capture the issues involved in matching "scientific explanations of the world with actual conditions in it":

First, do scientific researchers actually observe or measure what they think they are observing or measuring? This is the problem of internal validity. Second, to what extent are the abstract constructs and postulates generated, refined, or tested by scientific researchers applicable across groups? This addresses the issue of external validity. [emphasis mine] (LeCompte \& Goetz, 1982, p. 43) 


\section{Internal Validity}

Lincoln and Guba (1985) suggest several techniques for addressing problems of internal validity, or what they call the "credibility,"of research findings (p. 301). Four of these apply to this study: (1) activities to increase the probability of producing credible findings (prolonged engagement, persistent observation, triangulation); (2) peer debriefing to provide an external check on the inquiry process; (3) member checks to provide "for a direct test of findings and interpretations with the human sources from which they have come"; and (4) referential adequacy--an activity in which archived "raw" data is used to check preliminary findings (Lincoln \& Guba, 1985, p. 301).

(1) Activities to increase credibility. Prolonged engagement is based on investing enough time in the research setting to learn the "culture" or context in which the data occur, to provide the opportunity to test for misinformation resulting from researcher or respondent distortions, and to build trust and rapport (Lincoln \& Guba, 1985). My scholarly interest in LEC began taking form in the fall of 1995 , approximately one year after initially becoming involved. Over the last year and a half of my association with LEC has been modified to incorporate my researcher stance. The time invested provided me with the benefits of prolonged engagement: trust and rapport within the community and in-depth knowledge of the culture, language, norms and practices contributing to the research context (Ely et al, 1991). 
Prolonged engagement has provided me with the much needed understanding of LEC as a context in which the data are embedded, "rendering the inquirer open to the multiple influences ... the impinge upon the phenomenon being studied (Lincoln \& Guba, 1985).

Where prolonged engagement provided scope of understanding, persistent observation, also achieved through my participation in the research context, provided depth of understanding. This depth allowed distinctions to be made between salient, relevant data and those that while interesting, did not contribute to answering the research questions. The risk of "premature closure" was mitigated by generating alternative conclusions and explanations in log entries and conversations with peers.

Credibility of findings was also addressed by employing modes of triangulation in terms of sources and methods. Multiple sources of data was achieved through using course notes from many different courses (in some cases, two sets per course from different participants) and multiple interviews (including archived pilot study data reintroduced later in analysis). The use of different data gathering methods also contributed to credibility through triangulation, as this study relied on interviews, participant observation and document analysis.

(2) Peer debriefing. Peer debriefing is another technique to increase credibility of findings in which the researcher exposes him or herself and the research to a disinterested peer in order to explore questions, test tentative findings, address 
biases, test methodological decisions, and provide much needed catharsis and connection to express emotions and alleviate isolation. These goals were accomplished in bi-monthly group thesis meetings with Dr. Poulsen and five other graduate students working on thesis projects. Students took turns presenting data and tentative findings, posed methodological and theoretical questions, challenged each others' assumptions and leant a much needed ear--sometimes even a shoulder-when the loneliness, frustration and doubt threatened to eclipse our progress. Lincoln and Guba do not adequately define the term "disinterested," however, it is safe to say that while this group was interested that its members succeed, this did not prevent critical thinking and honest discourse.

(3) Member checks. Findings were checked periodically with a member of the LEC community to determine if emergent metaphors and conclusions about the study context and population accurately reflected the worldview of that community.

(4) Referential adequacy. Referential adequacy was accomplished for interviews and course notes by setting aside some data during analysis of these two data sets. Unanalyzed pilot study interview transcripts--secondary data--was "archived" to provide a method for creating "referential adequacy" for the interviews (Lincoln \& Guba, 1985). Two sets of course notes were also set aside during initial analysis of this data set. Archived data was not "recalled" until tentative findings had been generated from thesis data. Once recalled, the essentially "raw" data was used to test 
the validity of conclusions, concepts, metaphors and interpretations of the emerging findings.

\section{External Validity}

Concerns for external validity were addressed through earlier described processes of documentation. "Thick description" used in log entries and to contextualize analysis also contributed to the quality of the "data base that makes transferability [external validity] judgments possible on the part of potential appliers" (Lincoln \& Guba, 1985, p. 316).

\section{Pilot Study}

A qualitative pilot study was conducted in order to increase the reliability and validity of this research project by allowing me to practice my interviewing, observation and analytic skills; to develop an effective interview guide; and to enhance my ability to work as both researcher and participant in this cultural context.

Five in-depth interviews were completed, ranging in duration from 70 to 180 minutes. Judgment sampling allowed for the selection of interviewees with varying depth (number of courses) and breadth (number of years) of participation in LEC, in order to better test the flexibility of the interview guide and procedures (Babbie, 1992). Interviewee selection was mitigated by issues of convenience as I relied on my personal network of associations in LEC for recruitment purposes. All 
interviews were conducted with informed consent, protection of confidentiality, audio-recorded and transcribed.

Detailed field/course notes were recorded during my participation in a 10 week LEC seminar during which I practiced "making the familiar unfamiliar" (Lofland \& Lofland, 1995).

The interview guide began as moderately to highly structured and evolved into a more loosely structured topic guide allowing a more conversational, participatory approach that was more appropriate given my personal relationships with the participants. Analysis of the pilot data indicated that metaphor analysis would be a fruitful method for researching this setting.

\section{Data Analysis}

The process of metaphor analysis was conducted using the analytic procedures of coding and memoing, which were relied upon as tools for interacting with the data. Data collected were organized into two main "bodies" for analysis: (1) "Texts" representing the discourse of LEC as the source of the metaphoric system (course notes, promotional brochures, and miscellaneous organizational artifacts) and (2) "Texts" representing the discourse of LEC participants to represent the domain of the metaphoric system as lived and negotiated by the community participants (interview transcripts). Combining these sources reduced the likelihood of missing significant metaphors during analysis (Koch \& Deetz, 1981). Analysis in this study proceeded in three general stages: (1) analysis of participants' texts; (2) analysis of 
LEC texts; and (3) inter-relating findings from the two sets of data. Initial stages of metaphor analysis were conducted within each data body and the final stages involved comparing, contrasting and integrating findings from each body of data into the final product.

\section{Analytic Procedures}

\section{Coding \& Analytic Memos}

A code can be defined as "the word, or short set of words, you apply to the item of data .... These are labels that classify items of information pertinent to a topic, question, answer, or whatever" (Lofland \& Lofland, 1995, p. 186). A code is essentially a category of meaning assigned to some piece of data. Coding was used to identify salient conceptual themes (e.g. "sharing," "empowerment"), patterns, and domains of experience suggesting metaphoric structuring. Potential categories of metaphor (orientational, structural, ontological) were also coded. Codes were allowed to emerge from the data, guided heuristically by the theoretical framework, sensitizing concepts and "native" terms. Codes were the initiating and organizing concepts for most analytic memos.

Analytic memos are written records of small pieces of analysis in which the researcher describes, explores, captures hunches, makes connections between data, etc. Memos give form to the abstract thinking of analysis:

Analytic memos can be thought of as conversations with oneself about what has occurred in the research process, what has been learned, the insights this provides, and the leads these suggest for future action. These memos are 
written about entries in the log, and they themselves become part of the log. (Ely et al, 1991, p. 80)

Memos were used extensively throughout the analytic process. Each memo was organized around a particular piece of data, concept, theme, or puzzlement and carefully numbered and catalogued. This system aided in ongoing analysis, allowing cross-referencing and retrieving information with relative ease (Strauss \& Corbin, 1990).

\section{Metaphor Analysis: Stages and Procedures}

The approach to metaphor analysis taken in this study was grounded in Lakoff and Johnson's (1980) theoretical framework which constitutes the human conceptual system as metaphorically structured and defined. While their premises suggest a general approach to analysis, Lakoff and Johnson do not specify techniques, steps or processes for conducting such an analysis. For the purposes of this study, information on analytic process was drawn from Koch and Deetz (1981) and Deetz (1984) who developed and reported on a general set of steps aimed at conducting metaphor analysis congruent with Lakoff and Johnson's framework.

The four basic steps adapted from these articles include: (a) recording members' talk (documents already recorded); (b) isolating metaphoric linguistic expressions from the data; (c) sorting identified metaphoric linguistic expressions thematically and beginning to identify the metaphoric concepts being evidenced; followed by (d) repeated sorting of identified metaphors to reveal patterns or "clusters" forming 
coherent groups and working out the entailments of clusters to examine the experiential structures. These steps were used heuristically and adapted to accommodate the two main bodies of data.

\section{Stage One: Participant Texts}

The primary goal at this stage was to generate thematic categories or themes of talk in the interviews, rather than extracting metaphoric linguistic expressions and sorting them into clusters of metaphors. This decision emerged early in stage one as a result of two realizations. The first had to do with the breadth of interview data. The nature of the interview questions allowed for considerable range in the topics discussed, which resulted in a lack of conceptual unity. Consider the difference between asking people to tell you about their experiences with conflict vs. asking people to tell you about their experiences in the Forum or with LEC. The first question is likely to generate metaphoric linguistic expressions in the domain of conflict. The second question, asked in this study, generated a number of different concepts most salient to that individual's experiences. While all Forums cover the same basic material, different people seem to focus on different aspects of that material depending on their individual needs, interests, goals, intellectual development, etc. Because metaphoric linguistic expressions are uttered in relationship to some concept (e.g. domain of experience), interview data required analysis and coding of salient concepts before looking at metaphoric linguistic expressions. 
The second realization had to do with the "transformational" nature of LEC's work. LEC presents a host of new concepts and metaphors that must be negotiated or coordinated with the individual's existing conceptual structures. Therefore, interview responses were laced with numerous conventional metaphors that complicated extracting and sorting of those metaphors specific to LEC's metaphoric system. Since LEC's metaphoric system represents an alternation in socially constructed reality, different interviewees displayed different levels of participation in this "resocialization" process evidenced by their use and understanding of various LEC related concepts (Berger \& Luckmann, 1966). Thus, the result of stage one was a collection of concepts, themes, and categories of talk, as well as some initial metaphoric structuring of those concepts, themes and categories.

\section{Stage Two: LEC "Texts": Course Notes and Documents}

Stage two focused on constructing a more comprehensive picture of LEC's metaphoric system that could be reconciled with the interview data and followed more closely the four basic steps for metaphor analysis outlined previously. The first step--recording the discourse--was already accomplished. Five out of nine sets of course notes were typed up, the others left in handwritten form and organized in a notebook. Two sets of notes were left out of the first analytic pass and consulted later as a check on tentative findings. Five brochures were included in the analysis, as well as miscellaneous documents. 
Coding in this data set consisted of identifying both conceptual themes and metaphoric linguistic expressions within each set of notes or document individually. Memoing began with the process of isolating and extracting metaphoric linguistic expressions, which were then sorted and clustered thematically by the metaphoric vehicle or the experiential domain being structured. As the metaphorical concepts began to emerge, metaphoric entailments were explored. Stage two included performing a detailed explication of LEC's worldview which provided the experiential structure necessary for understanding and interpreting the emerging metaphors. The process of explicating the worldview also revealed LEC's rootmetaphor structure.

\section{Stage Three: Inter-relating and Integrating}

In the third stage of analysis, the emergent system of metaphors was compared with the categories and themes of talk generated from the interview data to explore if and how the metaphors, their entailments and experiential structures actually occurred in the discourse of cultural participants. 


\section{CHAPTER FOUR--FINDINGS:}

\section{EXPLICATION OF LEC'S WORLDVIEW}

The contents of both chapters four and five are devoted to findings generated during this study. Chapter four provides a detailed explication of LEC's worldview, as well as a rationale for this explication and a discussion of the relationship between metaphors and worldviews. Chapter five presents the emergent system of metaphors abstracted from analysis of LEC texts and interview transcripts.

\section{Metaphors and Worldviews}

Analysis of the present data suggested that participation in LEC courses involved processes of constructing and reframing domains of experience for many key concepts in this community and without knowledge of these culture-specific domains of experience, metaphoric mappings utilizing these domains would be difficult to interpret. It became clear that the ability to understand many of Landmark's emergent metaphors did in fact require some familiarity with this culture's worldview.

Within the framework of the conceptual theory of metaphor, any metaphor that occurs in speaking is not an isolated artifact of language; rather

each metaphor is a witness for the system of which it is a part. The appearance of a metaphor in speech at the same time is part of the production of the metaphorical system and reproduces the system that exists. The metaphors in this sense produce their own conditions for being understood. (Deetz, 1984, p. 219)

Metaphors producing and reproducing their own conditions for being understood 
has important implications for interpreting and understanding the metaphoric systems of different cultures. As discussed in chapter two, the understanding provided by a metaphor involves mapping knowledge about a source-domain of experience onto knowledge about a target-domain (Lakoff \& Johnson, 1980; Lakoff, 1993). The knowledge about experiential domains necessary for understanding a metaphor will tend to be culture-specific (Deetz, 1984) which suggests that interpreting metaphors of another culture requires familiarity with larger symbolic system and experiential context, in other words the paradigm, worldview or shared reality within which those metaphors operate.

A culture's worldview can be thought of as the extension of a metaphoric system's entailments and inference patterns (i.e. experiential structure): a paradigm of shared ways of thinking, experiencing, and acting in a culture that are "based upon grand systems of metaphors" (Deetz, 1984, p. 218). The terms "worldview" and "paradigm" are used interchangeably in this study to label an "implicit or explicit view of reality." (Morgan, 1980, p. 606). Guba and Lincoln (1994) describe a paradigm as "a set of basic beliefs" representing "a worldview that defines, for its holder, the nature of the 'world,' the individual's place in it, and the range of possible relationships to that world and its parts" (p. 107). Metaphors then, are at the very least, ways of understanding and enacting an aspect of a particular paradigm or worldview: "[M]etaphors are not neutral representations of reality; they are manifestations of particular ideologies and world-views, and have implications for 
what counts as information, and for what is thinkable" (Smith \& Eisenberg, 1987, p. 369). The concept of a "root-metaphor" is itself a metaphor that maps a relationship between metaphors and worldviews. A "root-metaphor" is a conventionalized symbolic frame, the roots from which a particular aspect of a worldview and an associated system of metaphorical concepts and metaphoric linguistic expressions "stems" (Smith \& Eisenberg, 1987). Since Lakoff and Johnson's (1980) theory of metaphor indicates that the "human conceptual system is metaphorically structured and defined" (p. 6), it is reasonable to consider that a worldview, as a coherent set of concepts for viewing the world, is thus, metaphorically structured and defined.

\section{LEC: A Paradigm-Shift}

The relationship between metaphors and worldviews (or paradigms) is particularly relevant to this study where the metaphors of interest are, arguably, both instrument for and product of a "paradigm-shift": an alteration of the organization, structure or concepts of one's worldview. Metaphors have been noted for their ability to create a perceptual "shift" of figure-ground relationships that can lead to a "transformation" in the way one views and acts in the world and to influence one's interpretations of and feelings about such a shift (Schon, 1993; Sackmann, 1989). LEC's courses are specifically designed to produce such a "fundamental shift" or "transformation" in the way human beings view and operate in the world:

We see that our "reality" may be held in place by our views, our experiences and beliefs, by the particular conversations we or others have about it. What takes place in the Forum is a transformation, a shift in our "reality," in what 
we already know. . . . This shift or transformation is not a one-time event, but a moment-to-moment approach to being alive--an approach that enables us to think and act outside existing views and limits and redefine ourselves and the reality we have known. When this transformation occurs, the world becomes framed in a new way--the way the world approaches us and the way we approach the world changes. The result is a new freedom and power in being, in creating who we are, and a fundamental shift in what we see and know as possible. (LEC Forum promotional material, 1996) [emphasis mine]

If indeed our conceptual system is metaphorically structured, then, as Lakoff and Johnson (1980) suggest, experiencing such a "transformation" or "paradigmshift" would be very much a matter of metaphor. It is my contention that the work of LEC is intended to "transform" or "shift" participants' "conventional" paradigm views through a process of reframing, reemphasizing and deemphasizing "conventional" concepts, as well as introducing new and unfamiliar concepts, and providing practical methods by which to integrate this new system of concepts into daily living. I adapt the term "conventional" from Lakoff and Johnson's (1980) claim that the human conceptual system is largely structured by a conventional system of metaphorical concepts, "that is, metaphors that structure the ordinary conceptual system of our culture" and that are "reflected in our language" (p. 139).

The term "conventional" as used here is intended to represent the dominant or mainstream U.S. culture worldview; the system of established meanings, views, practices, and customs that comprise the domain of our "primary socialization" (Berger and Luckmann, 1966). The term "conventional" also points to a contrast between conventional conceptual metaphors structuring the dominant worldview 
and the conceptual metaphors arising from LEC's intentionally crafted, alternative worldview. As a form of "paradigm shift" or "resocialization" (Berger and Luckmann, 1966), LEC's worldview is still informed by the conventional concepts of primary socialization; however, analysis reveals that many conventional concepts are reframed and reorganized as to their relationships with one another and the value attributed to them in this community.

\section{LEC's Way of Speaking: The Language of Transformation}

LEC's metaphors and worldview are also constituted and expressed in LEC's unique and distinctive "way of speaking." Part of participation in LEC's courses is being introduced to and developing the use of this way of speaking. The "language" associated with LEC is one of the factors that most distinguishes membership in this community. This way of speaking includes new words whose meanings are "distinguished" during courses, familiar words that are "reframed," as well as more subtle differences and patterns in the use of words, phrases, and linguistic structure. LEC's way of speaking also seems to have what one interviewee and I began calling the LEC "accent" or "vocal framework" (Ivan, 1803-18): recognizable patterns in vocal inflections, rhythms, cadence, and emphases in speaking. Members and nonmembers of this speech community point to the existence of this way of speaking with nicknames such as "Landmark-speak," "Forum-speak," and "Landmark-ese."

LEC's "technology" (the word they use to label the "body of distinctions" and practices that comprise the material of their courses) represents a "paradigm-shift"-- 
a "leap" to a "finite province of meaning" existing alongside or within "conventional" everyday reality (Berger \& Luckmann, 1966, p. 25). According to Berger and Luckmann, language, in imposing order "on the chaos of experience," both apprehends and produces the "common objectivations of everyday life" (pp. 37; 153). Participating in LEC's "new" paradigm entails new ways of thinking, speaking, being and acting that are potentially in tension with the common language of everyday life used to produce and access the "reality" of our experiences.

Putting the debate regarding linguistic determinism aside, it seems appropriate to consider Whorf's view that a way of speaking shapes the reality or worldview of a speech community (Whorf, 1956) ${ }^{2}$. If we inherit a language, a way of speaking, and thus, a particular worldview, it seems possible that a new way of speaking could actualize--may even be necessary for--the experience of a "paradigm-shift," of seeing the world and our reality in a new way. Indeed, language and social interaction (e.g. conversation) are the primary means by which subjective realities become "objectivated" as social realities and by which social realities are altered or transformed through "resocialization" (Berger \& Luckmann, 1966). One reported intent of LEC's particular "way of speaking" is, in fact, to have participants speak, and thus think and act, in new ways, in addition to focusing people's awareness on

${ }^{2}$ Heidegger also identified language as something human beings inherit from the culture or society in which they find themselves "thrown"; that is, we are essentially born into (thrown) a particular language or way of speaking, and that language constrains what we can experience and express (Mulhall, 1996. p. 94). 
their communication practices in general ${ }^{3}$ (AC jotted notes; FIA jotted notes; Carl, 1003-1091; Mora, 549-610, 1543-50). Any understanding of the everyday life of a social reality thus requires an understanding of its language (Berger \& Luckmann, 1966).

As stated earlier in this chapter, analysis revealed that familiarity with LEC's alternative worldview was necessary for interpretation and full appreciation of the metaphoric system that both reflects and structures it. Furthermore, the findings of this study are deeply embedded in the "way of speaking" shared by participants in this community. The intent of the following pages is to provide an explication of LEC's worldview and "way of speaking" to serve as a context for discussing the rest of the findings of this study. After a brief discussion of the ontologically-driven focus of LEC's courses, the worldview explication is presented using the categories offered by Guba and Lincoln (1994) in their worldview definition to provide a rough organization for these ideas: (1) The nature of the world; (2) the individual's place in the world; and (3) the range of possible relationships to that world and its parts.

\section{LEC's Worldview}

\section{Background: An Applied Ontology}

LEC's courses deal explicitly with issues of ontology: the nature of human existence and being and issues regarding reality. They also address issues of

\footnotetext{
${ }^{3}$ The significance of vocabulary and distinctions is reminiscent of Heidegger's opinion that the world available to us is "given" by the distinctions we draw in the particular vocabulary and way of speaking we use (Gottlieb, 1990).
} 
epistemology: what is thinkable, what is knowable, and how we know what we know. Although I have rarely heard a course leader cite a source of origin for any ideas presented, in my own research I have found what appear to be "traces" of their ontological approach in the work of Sarte and Heidegger, as well as the perspectives and methods of Gestalt therapy founded by Fritz and Laura Perls in the 1940's (Yontef, 1989) and the philosophies of Zen Buddhism. It is difficult to determine the extent to which these works have influenced LEC's work--what they have included from whom and where the ideas are parallel, deviate, etc. LEC seems to have woven together--cut and pasted--what they wanted from various traditions, sometimes building on an idea, sometimes using it as a place to step off from. My own inclusion of these ideas in the following discussion is in a similar cut-and-paste style; I have tried to indicate the links I see, but at times have left out these potential connections as the specific origins of LEC's worldview are not the primary focus of this study, nor are they necessary for the understanding of the analyses that follow.

I call LEC's model an "applied ontology." I use the term "applied ontology" to indicate my observation that LEC adapts and integrates what are essentially passive, static, and abstract philosophical traditions (with the exception of Gestalt therapy which may actually be part of what LEC has drawn upon to achieve "applicability" of their material) into an "applied" system of active, dynamic methods and practices LEC calls their "technology": "the application of scientific knowledge; the methods and materials of applied science" (Websters II, 1984, p. 705). When reading or 
discussing various traditions of ontological philosophy, it is easy to agree with or to note the relevance of some ontological premise or collection of premises, but much more difficult to integrate them into an actual model for living. This is what LEC has done: constructed a worldview informed by a working, applicable ontological model for living. What follows then, is an attempt to describe key aspects of the worldview emanating out of LEC's "applied ontological" approach to living and to explain key terms in the culture's way of speaking. ${ }^{4}$

\section{The Nature of the World}

\section{Life Is Empty and Meaningless}

In LEC's worldview the nature of the world (LEC uses the world "life") is "empty and meaningless," a metaphor that captures a familiar existential notion: that the world itself is without meaning. Not only is "life empty and meaningless, but "it is empty and meaningless that it is empty and meaningless." In other words, saying that life is empty and meaningless does not mean anything either, its just "what's so."

\footnotetext{
${ }^{4}$ Since LEC is an oral culture, I have little in the way of documented sources from which to construct this explication. I rely most heavily for accomplishing this explication on my own extensive experiences in this community, my course notes and research logs, and to a slightly lesser extent, my interview data. This can actually be considered a strength, since the source of the explication is from the perspective of one who actually participates in life, to a certain extent, from this worldview, rather than a complete outsider attempting to explain something only peripherally experienced (Ely et al, 1991; Lofland \& Lofland, 1995). While I strove to include the most "important" or fundamental elements, to make them accessible, and to articulate the relationships among the various aspects, I know there are places where I have left holes or created more questions than answers. But then, perhaps that is what this process is all about anyway: discovering what the questions are.
} 
LEC's meanings for this phrase (a meaning structure called a "distinction" in this community; see pp.113-118 for an explanation of this concept) are very similar to Sarte's existential position that "reality" prior to any form of human "intervention" such as apprehension through language use (what he called "being-in-itself") is without value or meaning of its own (Palmer, 1995). Human beings create meaning and value through their language, choices, actions. Thus, "right and wrong," "good and bad," "moral and immoral," "shoulds and shouldn'ts"--all these are human "inventions," although human beings tend to live "as if" their inventions were "real." According to LEC, "the Universe is enormously indifferent to the meaning we make of life" (AC, 150-51).

One of the jokes among those who have completed the Forum is that what they got for the price of their tuition and the investment of time was "nothing" (which of course, would not occur as very humorous to those unfamiliar with what happens during the Forum!). The claim that "life is empty and meaningless" leaves those who search for meaning in the world, for purpose in life, with "nothing" instead of something. This can be a very disturbing notion for a variety of reasons (for example, the existential "anguish" Sarte said accompanied the realization of our freedom and responsibility as the source of our meanings). In LEC, however, "nothing" is "possibility": the opportunity--and yes, responsibility--to choose meaning for ourselves. "Nothing" in this community means no limits and the freedom to choose from among any number of possible ways to be. Thus, life is like 
an empty vessel, and that very emptiness exists as the ultimate possibility. Similarly, "a possibility fulfilled is no longer possibility" (SELP notes).

\section{Two Worlds: What Happened and Interpretation}

Early in the Forum a crucial distinction is developed between the "world" of fact or "what happened" and the "world" of interpretation about "what happened" or "story." I label this distinction as "crucial" because the "logic" of this model appears to be the foundation for several other key aspects of LEC's "applied ontology." The model is depicted by drawing two circles, one labeled "what happened" and the other "interpretation." Typically using examples shared by the participants, the Forum leader distinguishes the difference between these two "worlds."

And so Richard just very calmly distinguished that what happened was a human being entered a room and said I would love to talk to you to the other human being in the room. And that human being said, "I can't talk to you and watch the Discovery channel at the same time." . . and her interpretation was "he's a jerk, he's a son-of-a-bitch, he doesn't love me". . . "He never listens to me," blah blah blah. And none of that happened: sob, jerk, never listens to me--that didn't happen in reality. Like you wouldn't find, "never listens to me" atoms anywhere in that room, it was just an interpretation that she had of the experiences that occurred. . . And, and, all throughout our lives we're interpretation-making-machines. Something happens and we interpret it, from a very particular perspective and point of view, etc., etc. But we take that interpretation for as, that's what really happened (Ivan 356-90)

In the realm of "what happened" are events that exist at the level of description (e.g. the car ran out of gas; the woman said "I would love to talk to you" and the man said, "I'm watching the discovery channel"). In the realm of "interpretation" are all the meanings human beings make about "what happened," what LEC calls the 
"story" about what happened (e.g. "I am so stupid for not noticing the gas gauge" or "I am so lazy for not stopping for gas an hour ago"; "He never wants to talk to me," "He doesn't care about me"). According to LEC, human beings tend to collapse the two worlds into one another and live like their interpretations are "real," rarely distinguishing their own choice and responsibility for the meaning they make of things. "Unreality is the collapse of 'what happened' with the story about 'what happened,' and living like the "story" is "what happened" (AC, 231-14). The goal is not to stop interpreting or acting on interpretations, but to develop an awareness of our own authorship and choice in the meaning we make of life. More will be said about this when exploring the "place of the individual in the world." The Nature of "Reality"

One of the major "conversations" in the Advanced Course is exploring the nature of reality. LEC's distinguishes reality as being of three types or levels, each with a corresponding human "test": personal "reality," interim "reality," and "ultimate reality." For the purposes of "translation" I might additionally label these three types of reality as subjective, socially constructed, and objective, with ultimate reality being as close as human beings can get to objective reality.

Personal reality and the personal test. Personal "reality" is that which is experienced by the individual. The "personal test" consists of thoughts, feelings, sensory experience, perceptions, inferences, opinions, past experience, etc. The personal test for reality consists of "checking-in" with ourselves--what do we think, 
feel, etc. LEC says that we tend to get "stuck" in the personal test, letting our thoughts and feelings tell us "how it is," which may interfere with one's ability to engage in effective action. Consider someone who wants to get a new job, but their personal test tells them "I don't have the right skills," or "they won't offer me enough money," and so they do not act. Or perhaps my personal test tells me that in the past, when I only had three weeks left to complete a major project I did not make it, so I could not possibly make the deadline on this project, and thus, I give up. These are simple examples, but hopefully they illustrate the personal test for reality. Of course LEC does not suggest that we summarily discount the personal test, which may occur as intuition, inner-knowing, or as an interpretation. The main point it that we often disempower ourselves unnecessarily when we remain at the level of the personal test. For LEC, those who experience themselves as "stuck" in life are likely stuck in the personal test.

Interim reality and the interim test. Interim reality is the form of reality created socially, among people. The substance of the interim test for reality is agreement. The more agreement that exists regarding something, the more "real" it is: that is, "agreement generates reality" (AC, 319). Beliefs, for example, are a system of agreement. "Belief is one level of knowing and the world is stuck at level of belief; the substance of belief is memory, which also exists in agreement" (AC, 313-14).

According to LEC, disagreement, like agreement, also contributes to interim reality. "The more I disagree, the more in fact I agree. In reality, disagreement is the 
same as agreement; in order to disbelieve/disagree, you first believe [or agree], then 'dis' it"(AC, 320-23). Some other aspects of interim reality are: repeatability, consistency, standards, predictability, authority, understanding, reasonableness, logic, tradition, rationality, and "normality." Human beings are said to give precedence to the interim test for reality, because we will "discount our personal experience based on agreement"; if human beings fail to get agreement regarding their personal test, they we will often invalidate the personal test (our experience) (AC, 303-4). This does not mean we should privilege our personal test either; The point is to recognize the power of agreement.

Ultimate reality and the ultimate test. Ultimate reality exists in the world of "what happened" (see discussion pp. 91-92): physical objects and events that we can describe, that have facticity. What is the ultimate test for reality? Physicalness: "1. exists in time; 2. covers a distance; 3. has form" (AC, 339-43)."The substance of physicalness is measurability; measurability is the ability to distinguish beginning, middle, and end; if you can note one of the three, you can infer the other two (AC, 412-15). The substance of measurability is TOTAL agreement" (AC, 579).

Another point made regarding the nature of reality is that "everything that is real exists, but not everything that exists is real" (AC, 329). For example, possibility (which is nothing) exists and isn't real, unless a possibility garners agreement and becomes "real." "Meaning exists and isn't real" (AC, 420-22). Infinity exists and isn't real--it is the medium. Silence isn't real and it exists--it is a context: sound and no 
sound exist in the medium of silence, but it is not measurable (no beginning, middle, end) (AC, 415-17). The "entire universe is beginning-middle-end interfacing with beginning-middle-end (AC, 570-71).

\section{The Individual's Place in the World}

\section{Meaning-Making-Machines}

LEC says that human beings are "meaning-making-machines," a metaphor designed to capture the inevitability of our role as the source of meaning in the world. Human beings cannot not make meaning, according to LEC because the process of meaning-making (i.e. interpretation) is automatic, a part of the design of human being. The individual also cannot control much of "what happens" in the world. Events that exist in the realm of "what happened" have what Sarte and others call "facticity" (Palmer, 1995; Berger \& Luckmann, 1966)--things that exist or have existed and that we cannot change or control. Some circumstances, according to LEC, are "rained" on us: "we are born into a variety of conversations" (AC, 34). What human beings do have control over are the interpretations we choose to represent experience, as well as our actions in the world; how and who we choose to be in the face of "facticity" or "circumstances," whether they are "rained" on us or of our own making. While we might possess control over how things "occur,"5 human beings live much of their lives without being aware of this control, which

${ }^{5} \mathrm{LEC}$ 's word to denote the potential interpretations arising for an individual during the process of perception. 
requires awareness of one's self as a meaning-maker. LEC's view regarding this is similar to the existential perspective in Gestalt therapy:

Most people operate in an unstated context of conventional thought that obscures or avoids acknowledging how the world is. This is especially true of one's relations in the world and one's choices. . . by becoming aware, one becomes able to choose and/or organize one's own existence in a meaningful manner. . . A Awareness is accompanied by owning, that is, the process of knowing one's control over, choice of, and responsibility for one's own behavior and feelings. (Yontef, 1989. p. 3, 11)

The process of meaning-making is understood in LEC through the metaphor of "conversation": someone speaking and someone listening, which includes intrapersonal communication (the significance of "conversation" will be discussed in an upcoming section and further discussed in chapter five). It is our "conversations," both public and private, that provide the context for "who we are being" in any given moment.

\section{The Being in Human Being}

LEC's work is said to be fundamentally about the being in human being. The way LEC conceptualizes human "being," there is no "distance" or difference between "who we are being" about something or someone and our "conversation" about that something or someone. My understanding of LEC's ontological position regarding "being" is that, essentially, "being" is a conversation and a "conversation" entails a way of being. This relates back to the distinction between "what happened" and "interpretation" discussed earlier. Our interpretations--the meaning we make in every moment of life--are "conversations" that we are "being." So, for example, if 
my "conversation" about my thesis process is "this is too hard," "I'm don't know how to do this," or "I'll never finish in time," I am also being "this is too hard," "I don't know how," or "I'll never finish in time." How do these "conversations" manifest in "being?" Well, perhaps I feel too tired to keep working, or maybe I get frustrated and quit, or I might "be" so worried about time that I actually waste time.

"Ivan," one of my interviewees, paraphrased a Forum leader as saying that "being a human being is being a conversation that's not distinguished as a conversation" (lines 442-45). This is to say that most or much of the time, human beings live like their "conversations" are "real," never distinguishing that they have a choice about "who they are being." Undistinguished ways of being are sometimes called "thrown" ways of being or "already always" conversations. There are two particular kinds of "thrown" or automatic ways of being LEC says human beings develop in their past and that "run" them in their lives, limiting their possibilities for being and action: "rackets" and "winning formulas."

"Rackets." A "racket" is described as "a persistent way of being, doing or having, plus a complaint" or "a fixed way of being plus a complaint" (AC notes). They can often be identified by a recurring complaint about something. "Rackets" are automatic way of beings described with an economic structure of "payoffs" and "costs." A "racket" is a particular kind of conversation and that "gives" a way of being with several possible "pay-offs": allowing a person to dominate or avoid domination, be right or make someone else wrong, and/or justify one's self or 
invalidate others. The idea that a "racket" has "payoffs" is why human beings "run rackets" at all. In its essence, a racket is a device to avoid responsibility: "Human nature is to avoid the domination of being responsible" (CAP notes, 9/96).

The "costs" of a racket are identified as things like love, intimacy, happiness, self-expression, relatedness, appreciation, respect, etc. The irony frequently noted when talking about "rackets" is that while we are avoiding domination or being right (the "payoffs"), we are also preventing ourselves from--incurring a self-inflicted cost--having the very thing we want in our life in the first place: rackets "rob us of an experience of satisfaction and joy" (http://www.landmarkeducation.com/forum/home.htm). Rackets, according to LEC, "just don't make a difference in the world."

"Winning Formulas." A "winning formula" is a way of being intended to compensate for a past "failure-to-be." This failure-to-be is identified as a formative experience typically occurring sometime in one's youth--"a time in life when you were just being fully yourself and something happened and there was a 'failure-tobe'" (AC, 203-10). According to LEC, developing "winning formulas" is an unavoidable part of the "being" of human being: "As human beings, we inherit that there will be a moment of 'something's wrong here'--there's no way to avoid it" (AC jotted notes, 11/95). This failure-to-be might be experienced as a "break in belonging," as if "something was wrong here," or when we experience ourselves as failing-to-be something or someone--smart, funny, wanted, loved, belonging, 
capable, etc. In the "conversation" one has about an experienced failure-to-be, a decision is made to "be" a particular way so as to avoid repeating the uncomfortable failure-to-be experience.

A"winning formula" then, "is a way of being in-order-to not be ". The blank is then filled with whatever it is we cannot be such as different or the same or boring or unlovable or stupid etc. This way of being is called a "winning formula" because it is a way of being that has us succeed or "win" in life. But because it is (1) automatic--note the metaphor of "formula"--and (2) always based on compensation, winning formulas are said to limit our possibilities in life, perhaps preventing us from seeing or creating more effective ways of being in difficult situations (Analytic memo 25 , lines 6-17). In addition, because "winning formula" is always driven by compensation, it is a way of being that is never experienced as fulfilling: "In that moment [the failure-to-be] we stop being and start becoming, but we never get there" (AC notes, 11/95).

"Rackets" and "winning formulas" are also ways of "not being present." In a "racket" one is not being present to one's own responsibility. In order to avoid responsibility human beings "be" a particular way and complain, rather than "be-atcause," or see one's self as the source of one's meanings and power. In "winning formula," an individual might experience a current situation as triggering the potential for repeating some version his or her "failure-to-be" experience; "winning formula" allows the individual to avoid "being present" to that possibility. "Rather 
than being present, we'll sell out because, once present, who is responsible?"

(Brkdwn, p. 52, line 8-9).

Both "rackets" and "winning formulas" are what LEC calls "thrown" ways of being. ${ }^{6} \mathrm{~A}$ "thrown" way of being is simply a way of being developed in our past that has become so "automatic," that we are "thrown," almost predisposed, to be that way. "Racket" and "winning formula" are automatic ways of being derived from the past; enacting them in the present is living out of what has been, not necessarily what is or could be. When these ways of being--these conversations--go undistinguished (not brought into conscious awareness), they are said to "run" us. Part of the purpose of the Forum is to have people distinguish their "rackets" and "winning formulas." Once they are distinguished, according to LEC, it is always in

${ }^{6} \mathrm{This}$ term is reminiscent of a word Heidegger used, although LEC uses it a bit differently. Heidegger used "thrown" to indicate the socially determined condition of Dasein's existence. in which any human being's ways of behaving are already given by the social environment, the culture--Dasein is completely shaped by his or her culture, which Dasein is born or "thrown" into. All Dasein's behaviors and all the possibilities for behavior have already been determined by Dasein's social environment (Lemay \& Pitts, 1994; Mulhall, 1996). This view is not compatible with LEC's worldview, in which one can step outside the "known" and create possibilities for living that are not determined by the past. Heidegger also called this environment one's existence, for existence is the "ground" from which being springs. "Human beings are constituted in terms of their environment," and the behaviors, such as "moving, thinking, speaking," "which make up our existence, are so basic that we never fully recognize their significance" (p. 45). This last piece especially sounds congruent with the notion of a "thrown" or undistinguished way of being. Because behaviors are "created within" human beings as they grow up in an environment, in response to experiences and situations, these behaviors "do not occur" to the human being, that is, the person is not aware of them, they go undistinguished. 
your power to recognize yourself "running a racket" or "being in winning formula mode." Awareness of these habitual, "thrown" ways of being provides the possibility and freedom to choose that or some other way of being. I often hear people who have completed LEC courses verbally acknowledging that they are "running a racket" or are "in winning formula mode," or are "inventing how I want to be about this" (Thesis log, lines 2271-76). "Rackets" and "winning formulas" do not get fixed, removed, or healed. They do not "go away," much to the dismay of many human beings! What one gains is the ability to "disengage" (my word) these automatic ways of being should one choose to do so.

Winning formulas and rackets are also not framed as "bad," "wrong," or negative ways of being, but as "possible ways to be." They are also comfortable and familiar--when in doubt, when faced with ambiguity, uncertainty, crises, etc., human beings will tend to be "thrown"--or throw themselves--to into one of these familiar, habitual, automatic ways of being (PN, Thesis $\log$, lines 2263-70). LEC says that people tend to bounce back and forth between these two "mechanisms," thus limiting what is possible because they provide limited ways of being/responding to situations. In the worldview that "life is a conversation," (this view to be discussed in more detail beginning p. 105) it follows that some conversations will be more freeing, others more constraining; rackets and winning formulas are viewed as constraining conversations. The goal is awareness which leads to the ability to distinguish in the present what is "giving you your being," responsibility, and choice. 
"Being" can be derived from mechanistic, automatic conversations or derived from distinguishing "what's so" and choosing the conversation that will give one being-even if that choice is a "racket."

\section{Responsibility: "Being-at-cause"}

LEC adopts the position that, in a world without inherent meaning, an individual has complete freedom to choose the meaning of his or her life and what actions to take in every moment of that life. Thus, the individual is ultimately responsible for the actions and meanings he or she chooses in life. While there is what Sarte called "facticity" in events that happen, this "facticity" cannot cause anything in the realm of human action (excluding involuntary reflexes or bodily functions) (Palmer, 1995). According to Sarte and congruent with LEC's model, getting caught up in what we perceive as the "necessities" of life--getting up in the morning, getting dressed, going to work, etc.--distracts us from seeing that such actions are actually just possibilities, and "only necessary relative to the goals" we have chosen (p. 69). The realization that the individual is ultimately the source of his or her own meanings and values reveals their freedom, not only in the meaning and value one chooses regarding "the past" and in the present, but also regarding the future. Responsibility regarding one's future can be the most confronting "because there is no facticity there. The future is yet to be constructed, and it is I who must construct it" (Palmer, 1995, p. 68). In LEC's worldview past-based meanings are chosen by us, and thus, can only influence the present and future by our choice. 
LEC courses go through a process of reframing "conventional" meanings and associations for the concept or "responsibility." Consider the course excerpts below addressing portions of this reframing participants "already always" meanings (meanings that are already there for us, derived from our culture and past experiences) for "responsibility":

burden, heavy, fault, praise, blame, credit, shame or guilt. All these include judgments and evaluations of good and bad, right and wrong, or better and worse. They are not responsibility. They are derived from a ground of being in which Self is considered to be a thing or an object rather than a context" (FIA notes, 3292; 3302-07). "Responsibility with "shoulds" is not freedom" as "shoulds" remove the experience of choice; "Enforced Responsibility is not responsibility. (AC, 3288-90).

Again I find a similarity to Gestalt therapy, which views responsibility as something distinct from the concept of "shoulds": "When people confuse responsibility with blaming and shoulds, they pressure and manipulate themselves; they 'try'. . . . In such instances their . . . choices in the situation are ignored and they overcomply or rebel against shoulds" (Yontef, 1989, p. 12). Similarly, LEC's version of responsibility is reframed as "freedom" and as a "context," a way of approaching the world, rather than a moral obligation or a condition to avoided:

Responsibility starts with the willingness to experience your self as cause in the matter. ... to deal with a situation from and with the point of view, whether at the moment realized or not, that you are the source of what you are, what you do, and what you have. This point of view extends to include even what is done to you and ultimately what another does to another. Ultimately, responsibility is a context, a context of Self-as-source for the content, i.e., for 'what is.' (AC, 3298-3315) 
Responsibility as a declaration of being cause in your world (AC, 3294-95) Responsibility is being able to declare one's self as cause in your life; $100 \%$ responsible, no room for making wrong. (AC, 3099-3101)

These conceptualizations of responsibility identify it as a source of individual power; declaring one's self as "cause" (a declaration in LEC has the power to create) locates power in the individual. Note that a declaration is expressed from a "willingness" on the part of the individual. While "being-at-cause" is not framed as a moral imperative in LEC, participation in this community would be difficult if one attempted to remain in a "powerless" or "victim" mentality. Making excuses or giving reasons, for example, is seen as giving one's power to the reasons: saying "'I am angry because' gives the reasons responsibility; every time you give power to a reason you give up your self" (AC, 461-62). This is not intended or portrayed to mean that when bad things happen to us it is our "fault," rather, that even in the face of unfortunate circumstances, indulging in reasons, excuses, and blame does not provide motives or intention for effective action. Deflecting responsibility in this manner is typically "a racket" and "rackets" according to LEC, "just don't make a difference."

One thing this sense of responsibility tends to create is the alleviation of blame, and thus, of defensiveness. I am not suggesting that all of a sudden people cease blaming others entirely, but I am suggesting that participation in this worldview makes it difficult for an individual to indulge in blaming and excuses for very long, that is, if I "buy into" the notion that I am the source of meaning, that I create reality 
in my speaking, etc. As we will begin to see in the next section, LEC's view about personal responsibility has implications for communication. If individuals create their own meaning out of what happens around them and what people say to them, then effective communication--the creation of shared meaning--becomes the responsibility of all participating in a conversation. For example, one practice toward effective communication in LEC is to "take responsibility for what people are left with after my conversations with them" (CAP notes).

\section{The Range of Possible Relationships to the World and its Parts}

\section{Language \& Communication}

Language and language use is identified in this community as the vehicle human beings use to create reality and the method by which we access everything in the world. The following quote is from a section of LEC's "home page" on the internet in which they describe "some of the ideas examined in the Forum":

Distinguishing Ourselves and Our World Through Language: We primarily exist in a world that arises in and is constituted by language. While we come to think of ourselves, our lives, the personalities we develop and the reality we know as set and fixed, The Forum proposes that our world and the reality we know is largely malleable and can actually be created and altered through language. (http://www.landmark-education.com/forum/home.htm)

LEC's position regarding language permeates every facet of their worldview.

Participants in LEC's courses develop an acute awareness of their own language use, especially as it relates to what reality they are creating or endorsing, and to whether or not they are empowering or disempowering themselves and others in their eyes. 
In addition to a heightened awareness of one's language use, participants develop a new appreciation of the role of communication--especially their own communication behaviors--in shaping their experiences in and of the world.

Old and new models of "communication". LEC identifies two models of communication, sometimes called "paradigms" of communication. In the "old paradigm," the language use of human beings is grounded in a view of reality where "words match the world" in a "word-to-world" model. In the "new paradigm" language use is viewed creating the world we experience, that is, the world matches the words we use in a "world-to-word" model (SELP, 172-73). LEC points out that the model of communication the majority of human kind operates from (what I have been calling the "conventional worldview") is a "word-to-world" model. In this model, people perceive themselves as using language to describe a world "out there" that, if described accurately enough, will make sense to the describer, giving them power to survive or prevail (AC, 114-16).

Even the way human beings learn language implies that first there is a world and then, in order to connect and deal with that world, we learn language. ... In this current paradigm, how we think of and know communication is essentially as a means to an end, a tool. ... the current paradigm in which we hold communication continues to reinforce itself and to keep the true nature of communication hidden from view. (Communication program brochure, 1994).

This "word-to-world" model would seem to be congruent with what Reddy (1993) identifies as the "conduit" metaphor shapes our speaking and perceptions regarding communication. 
LEC claims to operate from the second model or "new paradigm" of "communication": a "world-to-word" model, in which what we speak is the shape of the world. The world-to-word model is congruent with constructivist perspectives regarding communication, where reality is created or "arises" in language and its use in communication. That is, the existence of language itself does not create reality, but its use in "conversations," with ourself and with others, is what gives rise to the world we live in.

[LEC] poses that language is not merely a bridge, a response, or a way to deal with the world out there, but is in fact what allows for and brings into being that world in the first place. ... When this shift occurs, when we see language and communication as both that which gives rise to the world and also the instruments that give us access to everything in that world, it alters the very nature of what is possible in our lives. Communication and language come to be seen for what they really are, creative acts. Listening and speaking . . . become the instruments of our creation. [LEC] suggests that what it is to be human has its own domain, and that domain is one of language--of communication--of conversation. (Communication program brochure, 1994)

\section{Conversation}

LEC says that human beings live "in a world of conversation," which highlights the idea that the world of facticity is not really available to humans except through language, or more accurately for LEC, through "conversation." LEC's says that "life is a conversation," a position that privileges the dynamic relationships among speaking, listening, and language over any of these elements individually. Although LEC does use the word "communication," their concept of "conversation" tends to subsume everything that would normally fall within the domain of communication: 
language, speaking, listening, nonverbal elements, etc. The word "conversation" seems to emphasize the process aspect while the word communication seems to emphasize the experience of the purpose or outcome. A "conversation" in its most basic sense is "someone speaking" and "someone listening," which includes intrapersonal communication. Intrapersonal communication is of crucial importance in a world where the individual is the source of his or her own meanings. All relationships then, to the physical world and to other beings, exist in the form of conversation; a relationship is a particular conversation.

"Conversation" is probably the most central concept in LEC; it is the link between human "being" and everything else that comprises human existence. As suggested earlier, a conversation is a process of meaning-making that gives rise to particular ways of being. "Conversation" is of course, a conventional term but, in this speech community, it acquires more "currency" than it is typically imbued with in broader, mainstream culture. The word "conversation" in LEC is used in a variety of modes: to indicate an interaction with another person in the past, present or future; to indicate an internal, intrapersonal communication, which may or may not be shared with another (e.g. "I'm tired of being taken advantage of--but that's just my conversation"); to indicate a particular domain, topic or type of conversation and all that is associated with it (e.g. The Forum is a particular "conversation," distinct from other LEC courses in its purposes, topics, context, etc.); to indicate a "story" which is another conventional word with specific LEC meanings as a particular type 
of conversation: an enduring structure of interpretations given to some experience; basically any system of meanings, large or small, associated with something or someone can be called a "conversation." The Forum and the rest of LEC's curriculum are often called collectively a "conversation for possibility."

People who are "active" in or identify with this community tend to use the word "conversation" rather than "talk." That is, one would say: "I would like to have a conversation with you about ___ rather than "I want to talk to you about ___.." In fact, my observations suggest that while in an LEC environment, such as at the Center or in a course, people rarely use the word "talk" in the way people tend to in non-LEC circles. Why is this significant? Well, for one, "talking" seems to emphasize speaking, and in the LEC speech community, listening is emphasized, extensively practiced and highly valued. Listening in LEC is identified as more important to communication and the creation of meaning than language or speaking: "What's more important than what you say is how its heard; what gives our speaking power is listening!" (AC, 636-37; 645-46). LEC's worldview is congruent with the premises of the social construction of reality--even our "self" is at least in part produced and maintained through "conversation": "An identity might be selfcontained but our self is not; our self lives in the language of others--we exist in our relationships to others" (AC notes, 11/95); "What alters who you are is the listening that is out there" (SELP, 21). 
When I use the word "conversation" in this community I am invoking, in a sense, the LEC experiential domain for "conversation" and the inference patterns associated with this domain (Lakoff, 1993). That experiential domain recognizes "conversation" as the structure/process for creating reality, meaning, identity-indeed that all human experience is fundamentally tied to our conversations about our experiences. If I say I want to have a conversation with you, I carry with me, as a participant in the LEC speech community, the notion that conversations create our reality and that conversations can alter reality. Participants in this community also carry the value of listening and language use as being of central importance in any communicative act. The word "conversations," as mentioned earlier, also encompasses those conversations one has with one's self--intrapersonal communication--in which we give meaning through our interpretation of experience. "Conversation" is essentially human access to the most important aspects of living: everything from whether or not we feel like going to work to commitment in marriage are conversations we create. Adopting such a notion of communication suggests a variety of potentially empowering notions. It could also imply a certain impermanence or lack of responsibility on the part of human beings; after all, its only a conversation I created, right? There is a concept in LEC that partially addresses this potential for impermanence and irresponsibility: integrity.

\section{Integrity: Honoring Your Word As Yourself}

Integrity is one of the conventional concepts that LEC "reframes" within the 
context of its alternative worldview. We have already seen that in LEC's worldview, human beings, through the vehicle of language/conversation are the source of meaning in their world, both at the individual level (personal reality) and at the global level (interim reality) through the power of agreement (which also occurs in conversation). This places the human being in a position of some power. In a "world" where speaking creates reality and the individual is the source of meaning, "Real power is a function of integrity" (AC, 661). This also relates to the role of personal responsibility. "Being one's word" and "being-at-cause" would tend to focus the individual's attention on his or her actions and the consequences of those actions for themselves and others.

In the "conventional" paradigm however, speaking does not seem to be particularly valued overall. Consider some mainstream views about language and speaking: "rhetoric" as empty speech; "all talk and no action"; "I don't listen to anything he or she says anymore"; cynicism and suspicion regarding the speaking of political figures and leaders who seem to repeatedly say one thing and do another or have hidden agendas. Essentially we do not trust people's speaking, including our own. Consider the mainstream view regarding "New Year's Resolutions"--promises to ourselves that tend to go unfulfilled. In general, LEC would say that human beings "lack a strong relationship with their word," a lack of "integrity."

Integrity in LEC is framed as an "alignment" between what one says and what one does, between words and action. A common analogy used to illustrate integrity 
is to say that "you have two tongues: one in your mouth, and one your shoe"; integrity is when both tongues go in the same direction. In a worldview where speaking is reality such as LEC's, power is in "honoring your word as yourself" and recognizing that "your word is action, bringing forth, creating reality" (FIA, 307879). Since identity, too, is a conversation, the notion of integrity includes an "alignment of the network of conversations that you are" (FIA, 3141-42).

In practice, integrity is keeping one's word, that is, keeping or following through on one's promises. If one fails to fulfill a promise, to "be one's word," the way to follow through and be in integrity is to acknowledge it, to "clean it up" with whoever is affected or involved--even if it is just one's self. Being "out of integrity" is conceptualized as creating a "mess" that, if left unaddressed, "damages our listening for ourself and other's listening for us" (Brkdwn, p. 11, lines 9-13) and thus, diminishes our power. "Cleaning up a mess" involves "owning" the discrepancy (sometimes called "distance") between words and action, which allows one to move forward with a new commitment, realigned in integrity. This cleaning up process is an important element in seeing integrity as a process, rather than an ideal state that can never really be achieved or maintained. It is not expected that human beings will never be out of integrity, in fact, being a human being means it is inevitable that we will. "The key is to make a commitment and be your word--not just lips moving for the sake of talking" (AC, 626-27).

The power of language, then, is developed within the framework of 
"conversation" which places the actual power of language in a transaction of speaking and listening, with listening being valued as the source the power of language/speaking. In this model, even what I speak only to myself has the power to shape reality because intrapersonal communication is still someone speaking and someone listening. Thus, intrapersonal communication shapes the subjective reality of the individual and this subjective reality can become part of the "objectivations" of social reality through language, conversation and "agreement" (Berger \& Luckmann, 1966).

\section{Distinguishing and Distinctions}

The ability to distinguish "who we are being" or the "conversation" we are having at any given moment is identified as a source of power in LEC: "Power is a function of distinguishing--the more you can distinguish, the more access to power you have" (pilot log, p. 9, lines 32-34). The question "what's informing your about to be?" or "what are you present to" asks an individual to locate themselves in the moment and become self-reflexive. Pausing to distinguish "what's present" or to "get present" is locating one's self in the present moment to determine what "conversations" are "giving" one's being: focusing one's awareness on the moment at hand, the emotion at hand, the experience, the thought, the person, etc., in order to see what is there. When one is "not present," one is simply thinking about something other than where you are right now or about something other than the topic at hand (like thinking about how to impress somebody instead of listening to what they are 
saying). You are always present to something and learning to make choices about what to be present to is part of distinguishing.

Distinguishing can be thought of a conscious process of manipulating figureground relationships. Distinguishing is a process that shifts an individual's focus to the context, or shifts one's attention from figure to the ground. When something is distinguished it is suddenly there, its available, accessible, or it "occurs"--appears, arises from the chaos, emerges as the figure. LEC says that "What doesn't occur, gives what does occur" (AC, 74-75) which is a way of saying that what does not occur is the ground (i.e. background) for what does. Distinguishing is fundamental to occurring because it creates contrasts: "Occurring comes from contrast. Until it occurs for you it doesn't exist" (AC, 73-74). Distinguishing in LEC is also inventing: "To bring into existence is to invent. ... Each of us invents when we distinguish--now something exists that didn't before" (AC, 537-38). Distinguishing provides access to the conversational "context" or "ground" that is "giving" how an event in the present occurs. Context, a vitally influential aspect of any conversation, is defined as "circumstances in which an event occurs; the explanatory words and ideas surrounding a particular word or statement in discourse" (Websters' II, 1984. p. 154). The context for any conversation can be viewed at the micro-level, such as participants' inner-experience (feelings, reactions, memories, etc.) as well as the macro-level, which might include the socio-political or historical climate regarding some topic of conversation. LEC places particular emphasis on the inner- 
experiences of the individual as the context influencing meaning, being, and thus, behavior.

References to context frequently appear in LEC's linguistic expressions. The language used to frame context is reminiscent of Gestalt psychology and Gestalt therapy. ${ }^{7}$ For example, talking about the "ground of being" or one thing that is in the background "giving" the existence of another. "'Ground . . . has to do with that 'against which' something is viewed as a contrast, or 'out of which' something emerges" (Brownell, 1996, p. 2). That which is the "ground" is that which typically goes "undistinguished" in the language of LEC, and that which goes undistinguished does not occur (or exist). Occurring is a product of contrast and contrast requires awareness of both figure and ground. The relationship between the figure (the "object" of perceptual awareness) and the ground (the context) is meaning (Yontef, 1993). That is, the ground influences the meaning an individual attributes to the figure. Like Gestalt therapy, LEC emphasizes awareness of the background or context, as well as the figure, as crucial to achieving clarity about automatic behaviors and the possibility of freedom, responsibility and choice (Yontef, 1993).

I visualize the effect of distinguishing our "conversations" as the creation of a space between our "being/conversation" in the present moment and our action in the next. Sarte talked about a sort of space or a hole in being he called "nonbeing," a

${ }^{7}$ LEC appears to adopt some of the language and techniques of gestalt therapy--not all that surprising considering that they share existential and phenomenological perspectives. 
nothingness or discontinuity in being that makes free action (and thus choice) possible (Palmer, 1995). Sarte also believed that while the past has "facticity" (the world of "what happened" in LEC), nothing in the past can cause a human being to do anything in the present (or future) because human being is separated from its past and "facticity" by a "nothingness." In that nothingness human beings choose the meaning of facticity for themselves (Palmer, 1995). But, because this "nothingness" usually goes undistinguished by human beings, we tend to live as if meaning exists in the world, rather than as a human creation; as if things in our lives are necessary, rather than part of our choices. Living this way is what LEC calls "collapsing" the domains of "what happens" with our interpretations about "what happens" and living like our "stories" are real.

My speculation is that "distinguishing" actually creates Sarte's "nonbeing," "nothingness," or discontinuity for people. In LEC, nothingness is possibility, the absence of the limitations of necessity, what we know, what came before, etc. In this "nothingness" all possibilities exist. Without distinguishing this existential space, this "nothingness" (which is in LEC terms is pure "possibility"), our actions, our meanings, our interpretations do not "occur" as choices we make among possible choices. Distinguishing is the key practice in providing what LEC calls "access to distinctions in your life," "opening" "new" ways of being and acting. "Standing in possibility" is described as standing in an "opening." The terms "access," and "open" or "opening" occur frequently in both LEC texts and interview transcripts. In 
addition to being a central orientational concept in LEC, the terms "opening" and "access" may be a way of representing participants' experience of an "opening" created when they distinguish that discontinuity or "nothingness," giving them access to possibility, choice, and power. In the moment of distinguishing the possibility of choice, we have the power to choose. This seems to be at least one reason why LEC says that "power is a function of distinguishing." Perhaps distinguishing "what's present" in any given moment actually creates the discontinuity, draws attention to the nothingness, interrupts our perception of the flow of time and "illusion" of causality, such that we perceive ourselves as having the power to act freely. This is an example of why I have been calling LEC an "applied ontology": they seem to have provided a method, an application, for distinguishing or creating that abstract, existential space in which choice and free action is possible.

LEC calls the entirety of their work a "body of distinctions," and specific content areas are often referred to as "a body of distinctions" as well (e.g. "a body of distinctions regarding reality") (AC, 296-97). A human being is also a body of distinctions: "We are a body of distinctions that brings particular things to life; What gives what we hear is the body of distinctions that we are" (AC, 540-42). Each of the concepts, terms, or practices--all that comprises LEC's system of meanings are called "distinctions." A distinction is the result of distinguishing. Words and meanings are typically not "defined," they are "distinguished." A distinction, such as 
"sharing" or "commitment," (yes they are words, but in LEC they are distinctions) is more than what a word means. A distinction includes connotations, associated actions, practices, and contrasts with "conventional" paradigm uses of the distinctions. The process of distinguishing includes both introspection and speaking about what we see in relationship to a concept at hand--"getting present to" what it is or is not, how it occurs, what behaviors or thoughts accompany it, contrasts and comparisons, sharing examples and narratives, definitions, etymologies, what are the costs and pay offs involved, how to apply it, what possibilities exist in uncovering hidden assumptions, etc. The result of this process is a set of shared meanings, and arguably, values and practices, called a "distinction" that reveals or creates something that was not there before, and in doing so brings new clarity.

LEC says that once you "have" a distinction, it is always there for you: "The nature of distinctions is that once you get them, you always have access to them" (Creativity sem., jotted notes). "Getting" balance in riding a bicycle is a common example used to illustrate what a distinction is. Balance is an experiential distinction that provides access to riding a bicycle. Once you "have," it you always "have" it. Another example of an experiential distinction was given to me by Ivan in our interview. In the example he also highlights that making (or getting) a distinction also makes a "whole new world" available:

And the distinction of a baby, when they're first learning how to crawl, often times they'll go backwards first. And then all of a sudden they'll get that they can go forward, and in that moment, they've got a whole new world of 
transportation, and, and exploration available to them because they can suddenly use, their arms and legs to go forward. And that is a distinction. (Ivan, lines 174-82)

Distinctions and distinguishing are difficult to describe precisely, but they are at the heart of "learning to speak the language of transformation" (log, p. 8, lines 19-21) and learning to live life as a conversation.

\section{Oral Culture.}

The culture of participation associated with LEC functions as primarily an oral culture. Unlike other organizations and individuals providing trainings and lectures in the self-development genre, LEC sells no books, audio tapes, videos, computer media, or any other form of recorded materials. They purchase no commercial advertising of any kind, other than promotional brochures which, along with various flyers and photo-copied handouts of course homework assignments, are the only forms of printed material distributed. LEC does maintain a web-site on the internet that is structured to provide information about the organization and the courses, much of which is basically identical to information already available in their formal brochures. Observations conducted in one of LEC's Centers suggests an organizational culture that relies heavily on the oral mode of information transmission. However, printed material regarding policies, agreements, and procedures are also extensively relied upon. Reliance on an oral culture, especially in the "culture of participation," might serve "hidden" organizational purposes, such as creating reliance on the organization for ongoing interactions in order to maintain 
the new system of meanings, protection of ideas from being "stolen" by competitors, or preventing critical analyses of the ideas comprising their technology. On the other hand, the positive values of an oral culture appear to be congruent with LEC's constructivist perspective of conversation as the central, fundamentally creative act giving shape to human reality.

All LEC courses are designed as a modified conversation, ". . . an 'inquiry'--a focused dialogue between the instructor and the participants--into what is possible in being human" (Brochure, Sharing the Landmark Forum, 1994, p. 3). The process of distinguishing introduced above is an integral part of LEC's course design, said to be based on the method of Socratic dialogue. "Socrates believed that learning was a creative process, in which a teacher served as a midwife at the birth of his pupil's ideas, rather than as a nurse feeding an infant with a spoon" (Freeman \& Appel, 1970, p. 36). Like Socrates, LEC seems to be aware of the role of speech in facilitating active learning, memory, and ownership of ideas (Kallick, 1989). Course leaders pose questions, make provocative claims, and go through processes of distinguishing; participants stand to answer questions, pose their own, challenge claims, share stories and insights, express emotions. While one participant is interacting-conversing--with the leader, others are invited-- reminded--to listen with their own life in mind, to participate in the dialogue themselves through the individual standing (who serves as something of a metaphor for the other participants). When a participant asks a question, the Forum or course leader tends 
not to provide an answer, but leads them to find the answer for themselves with more questions and examples. LEC's dialogic method appears to be crucial to creating, "sedimenting" and maintaining the rich shared meanings and way of speaking of this community. Ongoing conversation with members of a social reality is also necessary for the symbolic system to "live" subjectively for the individual, especially when the reality in question is an "alternative" one requiring some degree of "re-socialization" (Berger \& Luckmann, 1966).

\section{Sharing}

Another important aspect in creating an "alternation" of realities is the development of strong "affective identifications" and "intense concentration of significant interactions" with those within the group or community of the alternate reality. In addition, the individual will need a way of interacting with those who exist as part of an individual's social network of relationships in the "conventional" reality. LEC's practice of "sharing" helps to address both of these needs, as well as the need for ongoing conversation to maintain the "living" presence of the alternative paradigm. "Sharing" as a practice involves talking with others about one's life: thoughts, feelings, challenges, experiences, breakdowns, breakthroughs, and in the context of LEC, one's "transformation"--what has "opened up" or occurred in one's life through participation in LEC. People are asked, reminded, encouraged, and "coached" (for exercises and homework) to "share" themselves. The practice of "sharing" is viewed as a conversation that creates openings, "movement," 
connections among people, and orients people in the "present."

"Withholding," on the other hand, is the opposite of "sharing." While sharing is contributing, withholding is "being stingy." Withholding is essentially "holding on" to conversations that disempower one's self and others, thus limiting possibility for one's self and/or others. Withholding is framed as barrier--something that keeps one stuck or constrains movement and can contribute to personal and relational discord: "Everything we are withholding keeps stuck" (AC, 328); "What ever stops you from sharing/being self is exactly what stops you in life" (AC, 449-50); "more withholding, more suffering (no relationship); more withholding, more stuck in private conversation--not present" (AC, 575-77). Withholding is more than not talking to people, it encompasses a quality of isolating our selves, often subtlety, from others. Withholding has its origins in our automatic ways of being--rackets and winning formulas--and other past-based conversations ("if I say that, she will get mad"; "He knows I love him, I don't need to say it"; "That will just make me look stupid," etc.). Like all of LEC's distinctions, neither sharing or withholding need be framed as right or wrong, good or bad--just possible ways to be. Even so, it is easy to see which of the two are more highly valued and normatively subscribed to in this community.

The concept of "sharing" may sound similar to the familiar communication concept "self-disclosure," however, there are some important differences. "Sharing" is not necessarily about revealing your personal life to everyone. "Sharing," like all 
LEC distinctions, has been imbued with a rich experiential domain particular to this community. "Sharing" is more than a practice, it is a quality, a willingness to share one's self with those around him or her as a "contribution" In fact, "sharing" is often referenced just that way: "sharing yourself." "Sharing" includes personal experiences, thoughts, observations, triumphs, "breakdowns" (LEC's word for occurrences that stop us in life--problems, challenges, etc.). "Sharing" is a vital element in the chemistry of this community, deeply integrated into the overall ideology and fulfilling social, personal and organizational goals.

The practice and value of "sharing" is developed during the courses and appears to be integral to how the work of LEC is accomplished in the large groups. Participants stand and share whatever is "there" to be shared and those who do not share get something from those who do. Hearing yourself, your thoughts, feelings, behaviors, experiences coming out of the mouth of another bridges the waters of isolation that separate human beings. As the course leader "coaches" the individual who is sharing, those participating in their seats get the benefit of the coaching as well. Sharing seems to facilitate feelings of safety, trust and acceptance as people experience themselves as being listened to and receive validation for their humanness.

A frequently reported experience of participants is profound shifts in how they experience other people and their relationship to other people. Sharing and the intense, often emotional, interaction during courses seems to create strong 
identification and a sense of connection among the participants who go through a course together. The sense of identification and connection is not limited to those who go through a Forum together, but seems to carry over to any with whom one shares the common experience of having been through the Forum.

Sharing, whether in a course or "out" in one's life, is framed as an opportunity to contribute and be contributed to, as conversation is the vehicle for creating relationship, "breakthroughs," community, etc.: "The background of relatedness is created through sharing and the more profound background of relatedness is, the more possibility for miraculous results" (AC, 179-81); "Sharing gives the quality of your life" (AC, 450-51). Contribution and being of service are two important values in this community and sharing is a particular type of conversation that enacts those values.

Recall the earlier discussion of the relationship between "being" and conversation--that conversations are what give us our being. Not just private conversations, but social conversations as well. Consider that someone else's conversation about me in part shapes my "self," since another's conversation shapes how they will "be" in relationship to me, which then shapes how I "show up" or "occur" for them, as weil as how I will "be" with that person. The conversation I have about someone (or something) is called my "listening" for them--similar in concept to the notion of "frame of reference." Our "self" then, "lives" in the listening of others; our "self" is composed of a network of conversations including 
conversations I have with myself about myself as well as those conversations I have with others and others have about me. LEC says we train our environment how to listen to us and when we alter who we are, such as after completing the Forum, people's listening for our "self" is still based in the past. Sharing in LEC's worldview becomes an integral part of creating and altering "who we are" for other people: "Access to altering the listening people are for who you are being is sharing. Not sharing 'in-order-to' or sharing 'about,' just sharing." (SELP, 120-22).

Congruent with the idea that "life is a conversation," transformation is a conversation as well, and sharing one's transformation is the key to keeping that transformation alive and present--through sharing we are said to create and recreate our transformation. Conversely, if one does not share their transformation, it said to recede (according to LEC you never lose it) and can only be generated by entering into the conversation again. Sharing one's transformation may take the form of "sharing the Forum." In this specific form of "sharing" people are encouraged to share with others in their lives what has opened up for them through their participation in LEC. Sharing the Forum often includes inviting people to guest events where they usually hear more sharing and explore what might be possible for them in doing the Forum and are given the opportunity (and yes, encouraged) to actually register. In this way, LEC relies on graduates "sharing the Forum" as their only real advertising and marketing.

The final feature of LEC's worldview to be discussed (although it is by no 
means the final feature that could be discussed) is the framing of temporal relationships.

\section{Past, Present, \& Future}

LEC appears to adopt the conventional metaphoric orientations of the past being behind us and the future being in front of us (Lakoff \& Johnson, 1980). However, LEC "alters the temporality of past-present-future" (AC notes) or the temporal relationships between these familiar aspects of time. LEC conceptualizes our past as being projected into our future such that we are "living into" some version of (reaction to, building on, avoidance) our past. "Human beings are given in the present by the future they are living into; the future we had [prior to the Forum] got determined by something we said in the past (there is something wrong here) and our future was to fix it!" (AC notes). Thus, human "being" in the present is being driven by decisions we made in the past about the future, what LEC calls "a sentence" we gave ourselves (e.g. "I'll never to that again," "Men can't be trusted," "I'm not a good student," "I don't belong"), as well as by "automatic" or "thrown" ways of being (discussed on pp. 96-101).

The goal of the Forum is to take the past out of the future which is achieved through processes of distinguishing our assumptions, our automatic ways of being and the stories or meanings we made up and have been living from. This leaves the future "open to being invented" in any number of possible ways. According to LEC, when you take the past out of the future, there is "nothing" there and "nothing is 
possibility." Because the "future gives us being in the present" (AC jotted notes), LEC has developed a practice of creating or "constituting one's self as a future as possibility."

The practice of "creating a future as a possibility" is intended to create, not only as a personal reality, but a possibility for all people to experience because "possibility isn't personal" (AC notes). For example, "dreams powerfully realized now," or "all people being profoundly exposed to the untamed beauty of God" both project a "future as a possibility" that can potentially be experienced by all people. These are intended to provide something that "calls you to be"--that is, provide a way of being consistent with living into that possibility, rather than a "racket" or some other way of being that limits possibility. Creating a future as a possibility does not insure one will wake up "being" their possibility. Rather, because human beings are "thrown" to be in particular ways, one has to choose what will call them to be and invoke it in speaking. A "future as possibility" is an example of creating a new conversation that is not limited by our past views of ourselves or the world we live in. Consider that if "living is a conversation," then the possibilities for living an extraordinary life are somewhat limited if you keep the conversation to yourself.

Having learned to distinguish past-based conversations and ways of being, one's being in the present moment is always available as a choice: choosing who and how one will be instead of letting that be determined by one's past. Choice is only possible if one is "present" to the conversations "giving being" in the present: 
"People can only express their power in life to the degree to which they are present to life; transformation begins with being present to what's so" (Brkdwn, p. 40, 2-3; p. 8, 39-40). Awareness and distinguishing are always occurring in the present, even if the content of awareness/distinguishing may be something from the past or a possibility for the future.

The worldview I have described as LEC's alternative paradigm is not revolutionary in the ideas it builds upon, but the integration and enactment of those ideas within this community as a cohesive model for living is. Drawing on Lincoln and Guba's (1994) categories of the dimensions of a worldview I have explicated LEC's version of the nature of the world, the individual's place in the world, and a sample of the possible relationships human beings have to the world and its parts. The intent of the preceding discussion was to provide a "working" familiarity with LEC's worldview to serve as a background against which to consider the metaphors yet to be discussed. In chapter five I move to a discussion of the root-metaphors and metaphoric system structuring and operating within LEC's worldview. 


\section{CHAPTER FIVE--FINDINGS:}

\section{EMERGENT SYSTEM OF METAPHORS}

Chapter five presents the root-metaphor structure that undergirds LEC worldview and goes on to present the emergent system of metaphors emanating from these root-metaphors to structure and organize the entirety of this worldview. The abstracted metaphors are organized into three thematic clusters or subsystems: conversation, being, and transformation, with a fourth section presenting LEC's system of orientational metaphors. The organization is arbitrary in the sense that all of the clusters of metaphors overlap with and depend on one another for their inference patterns. Excerpts of interview data are presented within each of these sections to illustrate how these metaphors are made meaningful by particpants in LEC's community.

\section{LEC's Root-Metaphors: A Worldview Triad}

The process of explicating LEC's worldview revealed a triad of root-metaphors that form the foundation for this worldview. A "root-metaphor" is a conventionalized symbolic frame, the roots from which a particular aspect of a worldview and an associated system of metaphorical concepts and metaphoric linguistic expressions "stems" (Smith \& Eisenberg, 1987). One of the identifying characteristics of root-metaphors is their "ability to undergird a broad area of meaning" capturing "a fundamental, underlying worldview" and providing" an inferential base for understanding more discrete attitudes and behavior" (Smith \& 
Eisenberg, 1987, p. 369). Root-metaphors typically operate below the level of consciousness and tend to be "unobtrusive" in terms of usage in everyday discourse. For example, the "conduit" metaphor Reddy (1993) identifies as shaping our views about and enactment of communication is not readily obvious unless you know what you are looking for. Neither is another common root-metaphor in our culture: "argument is war," (Lakoff \& Johnson, 1980). Over time these metaphors become assimilated, naturalized, and ordinary--conventional--to the point where it seems as if that way of seeing the world is just the way the world is.

LEC's root-metaphors, on the other hand, are quite obvious by comparison. They are clearly laid out as a foundation and developed systematically into an alternative worldview. These root-metaphors, or very obvious versions of them (in the form of linguistic expressions), do tend to appear regularly in the discourse among those who participate in this community. The rather brazen nature of LEC's root-metaphors makes sense when considered in the context of creating a paradigmshift and developing an active "sub-culture" informed by an alternative worldview.

My analysis suggests that the explicit nature of these root-metaphors is the result of three primary influences. The first is that this alternative worldview and "sub-culture" are being intentionally created, and thus, the new framework and its "logic" must be made readily accessible to those in the process of "conversion." The second is the fact that LEC represents a "dynamic" and "unstable" community in terms of membership. There is a Forum nearly every month in those cities with an 
LEC Center, average class size is around 100 participants. This means there are always new participants in the community. Thus, membership varies widely in terms of the depth (number of courses taken, degree of involvement) and breadth (number of years actively participating). The third major influence regarding the explicit nature of LEC's root-metaphors is the potential for ongoing "frame-conflict" (Reddy, 1993) between LEC's in many ways alternative worldview and the mainstream or more conventionalized dominant worldview. Members of this community may find they need to ongoingly negotiate between the two systems' different values, inferential bases, and conceptual structuring. Thus, the rootmetaphor structure and the metaphoric system that emanates from it must be readily accessible in order for LEC to address those factors.

LEC's triad of root-metaphors demonstrates a one-to-one correspondence to the three major categories Guba and Lincoln (1994) claim a worldview defines for its holder: (1) The nature of the world; (2) the individual's place in the world; and (3) the range of possible relationships to that world and its parts. These categories are once again adopted to organize the discussion of findings in this section. Each of the root-metaphors depends on the inference patterns of the other two for its own "logic," and thus, they form a coherent underlying system for LEC's worldview. The triadic structure increases the metaphors' ability to address both anticipated and unknown factors in the process of transformation (Sackmann, 1989). 
The Nature of the World:

\section{LIFE IS EMPTY AND MEANINGLESS}

This existential metaphor is an adaption of the conventional ontological metaphor LIFE IS A CONTAINER (Lakoff \& Johnson, 1980). The conventional use of LIFE IS A CONTAINER suggests an individual's life is a container he or she fills ("I've had a full life," "Live your life to the fullest"). In the conventional framing, life is also a container already full that one removes meaning from ("Get the most out of life," "There's not much left for him in life"). In these versions "life" exists with or contains the substance of meaning, while human beings "discover" it, "accrue" it, and act or fail to act on it.

LEC's adaption of this conventional metaphor refers both to the totality or existence of life (the world) as well as an individual's life. In either case, the container is fundamentally empty of the substance human beings so often look to discover in it or from it: meaning and/or purpose. Metaphoric linguistic expressions sanctioned by this root-metaphor indicate a distinction being made between life/the world and meaning/language:

$\square$ There is life, and the meaning we give it; there is nothing and language. (AC notes 11/95)

$\square$ The Universe is enormously indifferent to the meaning we make of life. (AC 150-51)

$\square$ "What's so" is never a problem. (AC, 286)

$\square$ Nothing is inherently anything, except by agreement; we live as if something is inherent. (AC, 327)

$\square$ Meaning exists, and isn't real. (AC, 422) 
The world doesn't operate linearly or causally, but because we have memory, we think it does. , AC 432)

The root-metaphor LIFE IS EMPTY AND MEANINGLESS does not infer that there is no meaning, only that meaning is not part of the facticity of life; life just is. The fact that life is "empty" represents "possibility" and freedom in LEC. Recall from the worldview discussion that "life is empty and meaningless and it's empty and meaningless that it's empty and meaningless." This rather redundant wording is intended to emphasize that human beings, upon hearing that life is empty and meaningless, will make that statement mean something (e.g. life is pointless, there is no God, I have freedom, etc.). Meaning, as suggested in the linguistic expressions above, is part of the domain of human "being," the subject of the next rootmetaphor.

The Place of the Individual:

\section{HUMAN BEINGS ARE MEANING-MAKING MACHINES}

This structural metaphor maps the domain of human "being" with the domain of "machines" and mechanical processes. The mapping of human beings or some aspect of human being as machine-like can be found in conventional metaphors such as THE MIND IS A MACHINE ("He just broke down," "Her wheels are turning" and IDEAS ARE PRODUCTS ("He produces ideas at an incredible rate," "Her intellectual productivity has decreased"). LEC's modification of these conventional notions suggests that manufacturing meaning is a fundamental aspect of being 
human. The process of meaning-making is considered automatic and inevitable:

human beings cannot not make meaning. They can, however, choose what meaning to make--an inference pattern derived from the entailment that machines can be automatically or manually operated.

$\square$... it is not what happened(s) in your life that has power, but what you make it mean. (AC, 216-17)

$\square$ Can't not make meaning. No absence of meaning. (AC, 374)

$\square$ You are always changing what people do or say into something else. (AC, 513-14)

$\square$ To the degree you can generate other interpretations, to that degree you have possibility; interpret facts anyway--tragic, silly, etc.--none are true; make up an interpretation that empowers you." (AC, 673-75)

$\square$ You are never upset over what someone said. (AC, 373)

$\square$ What uses us is the meaning we assign to it, what we make up about it. (Brkdwn, p. 63, 31-40)

Comparing the linguistic expressions here with those associated with LIFE IS EMPTY AND MEANINGLESS above, suggests an interdependence of these two root-metaphors. That is, without the foundation of the metaphor LIFE IS EMPTY AND MEANINGLESS, saying that HUMAN BEINGS ARE MEANINGMAKING MACHINES would not provide any new way to approach the world or ourselves in the word. But because "life is empty and meaningless," what ever meaning human beings make $i s$ what things mean.

If human beings are machines that produce meaning, what is the raw material used by the machines to manufacture their product and by what process does the manufacturing take place? The inference patterns of this and the previous root- 
metaphor combine with the next to frame language as the raw material for meaningmaking and conversation as the process by which meaning and being is produced.

The Range of Possible Relationships to the World:

\section{LIFE (LIVING) IS A CONVERSATION}

At first glance this metaphor might seem to be at odds with saying that "life is empty and meaningless." How can life be empty and meaningless and a conversation? Does that suggest that a conversation is empty and meaningless? The two metaphors seem to structure different aspects of the concept "life," although the necessary distinction is not immediately available in the words of the metaphors. The metaphor LIFE IS EMPTY AND MEANINGLESS is a static metaphor in that it refers more to the domain of existence and events: what we can observe, describe, measure--the factual. Life is a container that we--human beings--"be" within and part of our "being" is to make our existence meaningful. Similar to some existential or contructivist philosophies, LEC describes human life/being as inseparable from language and language as inseparable from meaning. Human beings, then, "live in a world of conversation" (Brkdwn notes) that occurs within the empty container of life. The root-metaphor LIFE IS A CONVERSATION structures the processes of living and "being" which are inseparable from "meaning-making."

$\square$ There may be a reality that exists outside of language, but we have no access to it. (AC, 586-87)

$\square$ The realities and circumstances of our lives really exist only as a product of the conversations that we are. (Communication Prg. brochure, 1996) 
$\square$ The quality of your life is a function of the conversation you are dwelling in. $(\mathrm{AC}, 483-4)$

$\square$... what I call my life is the survival of some meaning; where you find the meaning is in the words, the story. (AC, 147-49)

$\checkmark$ What upsets us about problems or anything else, is the conversation we have with ourselves about it. (Brkdwn, p. 63. line 31-32)

$\square$ Being a man or woman is a conversation based on plumbing. (AC, 33)

Recall the distinction LEC makes between the "old" and "new" paradigms of communication. In the old paradigm, human beings use words to label and describe a reality that exists "out there." This statement summarizes the predominant conventional, "conduit" influenced framework for communication. In the "new paradigm," "life is a conversation" and the common place, ordinary activities of speaking and listening become life-giving and life-sustaining activities. In this system of metaphors, "conversation" is process and product: meaning is produced through conversation and meaning is a conversation. This emphasizes the idea that the world of human beings is inseparable from language.

The metaphors of LEC's system are not based on new ideas. For example, saying that "life is empty and meaningless" is itself not a new idea, nor is the notion that "life is a container" new. Saying that "life is empty and meaningless" conventionally speaking, might stir connotations of nihilism or atheism. Conventional notions about "life as container" tend to frame meaning as a positive and desirable quality (or substance if we stay with the metaphor's entailments). In LEC, however, "no meaning" is not a negative condition, but one of pure possibility: "In the Forum, the last day that, you know, that life is empty and meaningless and 
what you get out of the Forum is you pay two hundred and ninety dollars for nothing, you get nothing, which is possibility" (Ivan, 1138-1141). Thus, "empty and meaningless" or "freedom from significance," is a desirable condition in this community, which represents a reversal of conventionally held values in mainstream culture.

The newness and power of these metaphors comes more from their systematic interaction, from the new way of understanding and approaching our experiences and realities as human beings that the system underpins, than any metaphor alone. For example, the comments made by the interviewee below suggest the interaction of the three root-metaphors when she notes that (1) there is no inherent meaning in the world; (2) human beings make meaning and can choose; and (3) the meaning human beings make is made through speaking--"however you say they're going to be." Also apparent in this excerpt is the experience of empowerment this individual seems to derive from the combined inference patterns of the root-metaphor system.

Life is empty and meaningless? . . what that meant to me is nothing has an inherent meaning. So, if nothing has a meaning in itself, you can make it mean whatever you want, and just how much freedom there is in that. . . [I]f you're going to live in a reality where nothing has an inherent meaning and you can pick any meaning you want for any action, any object, any thing in the world, then there's total freedom there for things to be however you. say they're going to be; for reality to be however you say its going to be ... you have total power over how your life is going to be vs. it happening to you, you know? (Jenna, 697-721)

New metaphors "can give new meaning to our pasts, to our daily activities, and to what we know and believe" (Lakoff \& Johnson, 1980. p. 139). For those who are 
not members of this community, however, these root-metaphors may seem strange, absurd, confusing, depressing, or perhaps even threatening. They may not provide new understanding or make new ways of being and acting available. This is, at least in part, because LEC represents a unique culture and speech community sharing a culture-specific symbolic system and set of experiences. The meaning a new metaphor (or any metaphor) will have for a person will be determined partly by culture and partly by past experiences:

The cultural differences can be enormous because each of the concepts in [a] metaphor . . can vary widely from culture to culture. ... There will also be differences within a culture based on how individuals differ in their views of the source and target domain concepts. (Lakoff \& Johnson, 1980, p. 142)

As demonstrated in the worldview section, LEC is a cultural context with a highly developed system of shared meanings providing the necessary experiential domains for interpreting their metaphors. For example, even within our own U.S. culture, the word "conversation". will mean something different to a communication scholar than it would to a nuclear physicist. The words used in Landmark's metaphors will be familiar to English speakers and may generate both positive and negative connotations. Members of LEC's community, however, have been through an interactive process of reframing and building new shared meanings for the words that constitute their metaphors and for the inferences and actions implied by them. Through participation in LEC courses, the cultural members have developed collective experiences and a unique speech community in which these metaphors 
provide actual strategies for making sense of and acting in the world. Thus, the particular usefulness and/or intent of these metaphors is in part contingent upon the shared meanings of the worldview that they structure.

\section{LEC's Emergent System of Metaphors}

The root-metaphor structure, as the foundation of LEC's worldview, is also the foundation for the system of metaphors embedded in that worldview. The influence of the root-metaphors is woven throughout the system of emergent metaphors presented here. These metaphors are discussed in four sections: conversation, being, transformation, and orientational metaphors.

\section{Conversation}

LEC's root-metaphor LIFE (LIVING) IS A CONVERSATION touches every aspect of their worldview. All other metaphors in Landmark's overall system are in some way related to or dependent upon the "conversation" metaphor. Two clear and coherently related clusters of metaphors regarding conversation emerged during analysis: "conversation as creation" and "conversations as structures."

\section{Conversation as Creation}

Congruent with the LEC concept that life and living are conversations, and with their constructivist tendencies regarding communication (see pp. 105-110 for a review of this aspect of their worldview), LEC's system of metaphors in this domain consistently frame language and communication as instrumental in creating and sustaining meanings and realities. Consider the "family resemblances" (Wittgenstein 
in Schulte, 1992) among the following key words (and their definitions taken from Webster's II, 1984) abstracted from metaphoric linguistic expressions regarding speaking, listening, meaning, communication and conversation. The family resemblances are indicated by the boldface type

Arise: to come into existence: originate; to ascend, move upward (p. 40).

$\square$ Being: existence; one that lives, esp. a person; one's basic or essential nature: essence (p. 65).

$\square$ Create: to bring into being; to give rise to (166).

$\square$ Exists: to have being or actuality; to have life: live; to occur (p. 245).

$\square$ Generate: to bring into existence, esp. by physical or chemical process: create (p. 292)

$\square$ Give(s)/Given by: to convey; to bestow: grant; to cause to have or be subject to; to bring forth: produce; to furnish: provide; to cause to take place ( $\mathrm{p}$. 296-97).

$\square \quad$ Invent: to produce or devise first: originate; to make up, concoct (p. 371).

- L Live(s)/Life: to be or continue to be alive; having life: living; to reside; the property by which living organisms are distinguished from dead organisms or inanimate matter (pp. 404; 409)

$\square$ Make: to cause to happen or exist; to bring into being: create or fashion; to cause to be or become; to institute or establish: enact; an act or process of making; to invent: fabricate (p. 421)

$\square$ Occur: to come about, to be found: appear; to come to mind (p. 486).

The family resemblances among these words frame communication as an act, instrument or process of creation. The products of creation--reality, possibility, interpretations--are attributed with the qualities of entities. Unlike the conduit metaphor that tends to frame meanings as static or inanimate objects to be inserted into words and transferred between people (e.g. "Don't force your meaning into the wrong words"), LEC's linguistic expressions, incorporating the words above, 
suggest that meanings are human creations, brought into being and sustained through/in conversation (speaking and listening).

In the creation metaphor cluster, conversation entails speaking, listening and language as part of the creative process, which together create, give life, or bring the world into existence. Speaking tends to be framed as an initiating act that generates life, while listening is instrumental in sustaining what was created. The metaphors below indicate the relationships among those different components of the process.

\section{COMMUNICATION/CONVERSATION IS A CREATIVE FORCE}

$\square$ inventing a new conversation acts as a declaration and a declaration gives life, it creates. (Brkdwn, p. 63, 41-43)

- We generate unlimited possibilities through communication as that which gives life. (LEC Assisting Charter, 1994)

$\square$ It is through language and conversation that we come alive. . . True communication is creation. It moves people. It generates experience in others. . True communication transforms both the speaker and listener. (Comm. prg. brochure, 1996)

\section{LANGUAGE IS A CREATIVE MEDIUM or CONTEXT}

\section{LANGUAGE IS AN ENTITY IN A CREATIVE PROCESS}

$\square$ Reality is given by language. Existence is given by language. (AC jotted notes)

$\square$ Ideals occur, arise in language. (Brkdwn notes)

$\square$ Self is a relationship that arises in language. (Brkdwn notes)

$\square$ We need to distinguish who we are, invent who we are, which arises in language--distinctions arise in language. (AC notes)

$\square$ The project occurs in your speaking. (SELP, 170)

$\square$ Vocabulary creates reality. (SELP, 38)

$\square$... consider that language may in fact be what brings that very world into being. (Comm. Program brochure, 1994)

$\square$ This program poses that language ... is in fact what allows for and brings into being that world in the first place. (Comm. Program brochure, 1994) 
SPEAKING IS A CREATIVE ACT; SPEAKING GIVES BIRTH TO REALITY

- Your word as action, bringing forth, creating reality.

$\square$ Secret is to speak possibility and bring it into reality.(AC lines 635-37)

$\square$ Possibility exists only when we speak it." (Brkdwn, jotted notes)

口 Speak the commitment into existence, otherwise its just a "someday." (SELP lines 147-48)

ㄱ. You create a future with everything that you say, so, what future are you creating? (SELP, 239-40)

\section{LISTENING IS AN ENTITY IN A CREATIVE PROCESS}

\section{LISTENING IS A LOCATION THAT SUSTAINS SPEAKING}

$\square$ We are a body of distinctions--that brings particular thing to life; What gives what we hear is the body of distinctions that we are." (AC, 540-42)

- I speak, no existence; committed listening creates an existence system." ( AC, 464-76)

$\square$ What gives our speaking power is listening! (AC, 645)

$\square$... someone speaking, everything else occurs in listening. (AC, 592-3)

$\square$ Speaking has its roots in listening. (CAP, jotted notes)

\section{SPEAKING AND LISTENING ARE THE TOOLS OF CREATION}

$\square$ Listening and speaking--actions that we would normally think of as ordinary and commonplace--take on new dimensions and unexpected power. They become the instruments of our creation. (Comm prg. brochure, 1994)

$\square$ Rarely do we treat speaking or listening as if they had that kind of weight and impact, or even as if they had the possibility of that which can bring something into being. (Comm prg. brochure, 1996).

- We generate ourselves every time we listen and speak to others. (CAP)

- Your listening alters the way another occurs for you. (Relationships)

The cluster of metaphors above represent a system of structural metaphors

regarding the process of communication (or conversation). Landmark is certainly

not the first to approach or present communication as a creative act or creative

power. However, in an English speaking culture that is pervasively influenced by the

"conduit" root-metaphor for communication (Reddy, 1993), LEC's well-developed 
and coherent framework occurs as fairly unusual. Let us consider what approaching the world from this model might provide or prevent.

What sort of experiential structure is provided by framing the elements and process of communication as creation? Communication, like creativity, might be seen as coming from within and having outward expressions that are enacted with some degree of intentionality. Language as a medium becomes flexible, capable of being used in a variety of ways for a variety of purposes. Like paint or musical notes, language becomes the possibility for creative expression, rather than objects to be arranged correctly to transfer a message. Communicating from the "creation" paradigm would seem to focus awareness on both the process and the product or goal in new ways. Since creativity is an expression from within, viewing communication this way might facilitate individuals being more aware of their own creations of meaning and impact on the creative process, as well as on the interaction between members (and elements of the process). A creative process requires freedom of expression, experimentation, and an openness to the possibility that the product may not look like one would expect it to. In a creative act or process, one's attention, ideally, is focused on the present moment, not on how well it will turn out or what it will look like. When something is created, there might be a desire to share it with others.

In addition, many of the metaphors in this system suggest that "life" is being created between the partners of speaking and listening, which connotes 
responsibility accompanying those acts and the product of those acts, and emphasizes the necessary interaction between members of the creative act (i.e. speaking and listening). That which defines success and failure of the process and product becomes more permeable. For example, a disappointing painting may lead to a new exciting expression.

What does this framework obscure about communication? One thing these metaphors do not provide for are communicative situations in which the other participants do not, for whatever reason, choose to enact communication in this way. If I approach a conversation as a moment of creation and the other is expecting me to extract meanings dutifully from the word packages sent to me, the creative process would seem to be hampered. Another thing not really, or at least not explicitly, addressed is the role of nonverbal and non-intentional forms of communication in the creative process. Yet another aspect not explicitly accounted for in the "communication as creation" cluster is the extent to which destruction, as the "opposite" of creation, plays a role in processes of communication. There is an implied responsibility for being aware of communication's power, but the thrust of these metaphors and the tendency to avoid "making something wrong" in this worldview may result in an ambiguity regarding what is or is not "appropriate" or desired.

Conversation as creation: Interview data. Looking at excerpts from the interview data reveals many of the potential aspects of such an experiential structure 
being realized, or least manifesting in the discourse of the participants interviewed. The "conversation as creation" metaphor appeared to be adopted in the speaking of many of the interviewees, suggesting that they experience it as useful, accurate or descriptive of their actual experience of communication. That is, the metaphor fits their experience. According to Lakoff and Johnson (1980) if we begin to experience a metaphor as "true" and base our actions on it, the metaphor will modify or become integrated into one's conceptual system.

The following excerpts highlight four sub-themes that emerge in the interview data regarding the larger metaphor of "conversation as creation." Some excerpts highlight just one or two, while other excerpts highlight all four. At first glance, however, these themes may not be apparent. The process of observing metaphors, be it structures or systems, is a process of observing a pattern; thus by reading through a series of excerpts about conversation the themes emerge. Another reason the themes may not be immediately apparent is that one must read cross-culturally. In other words, even with explanation the metaphors and meanings of a logging community or of the N.W. "grunge" culture may not be readily accessible to those not from that culture, unless the observer is carefully looking for a pattern. Having argued that LEC is a community that holds an atypical worldview, it is logical that the language and expressions of its members may occur as foreign to those outside the culture, especially where metaphorical themes are concerned. 
Wittingly or unwittingly, the interviewees share expressions, realizations, and observations revealing entailments and inference patterns regarding the larger metaphor of "conversation as creation." Analysis of interview data reveals four prevalent "sub-themes": (1) a heightened awareness of one's own attitudes about communication and actual communicative behaviors and how those attitudes and behaviors impact the outcomes and experience of the communication process; (2) a constructivist perspective expressed as a realized connection between language/conversation and the creation of realities; (3) an awareness of the possibilities available in approaching communication/conversation as an act of creation; and (4) a conscious awareness of one's personal responsibility and choice, as well as an experience of personal empowerment, associated with communication as a creative act.

I just hear things different. I just. . . Isn't it funny, you know, nothing really changes, its just all in your head, between your ears, and you just hear it all different. (Leigh 154-57)

$\square$ But when you're paying attention to this person, and really, trying to appreciate them and getting--being completely present to the idea that they're expressing it just, elevates the possibility of what can come out of that into something ... beyond what you know now, you know. And that can only happen, I--I believe that it can only happen--when you are totally present and totally paying attention in a completely nonjudgmental way and just saying, like, anything can happen from this conversation that I'm having with you right now, you know. And that was just, amazing. The possibility of the words that you choose and the language you use to make the future happen. (Mora 663-682)

- And what I notice is in conversations with people, I'm not listening to what they're saying, I'm listening to what I'm gonna say next in my head, and I don't even hear them. And that's the way I am most of the time--because I've got a lot 
of practice being that way. But I'm really making it a point in my life to choose to listen to people, because that is what I want to do. . . but its real exciting I would say that I do see myself choosing that more often now and its very, very rewarding. Its very exciting to me . . when I do that I love all those people, no matter what I thought about them before . . . . You know, they're just perfect-they should be that way. Versus, god, why is he like that; he's holding us back. (Jenna 862-885)

... the possibility for the future that you create is generated by you. 'Kay, absolutely, once you know, the possibility of it, you generate it and other people can help you, and then, so, it is in your control that you generate something, through speaking. And then that didn't happen in that particular case. I didn't have the in--the flash of inspiration to generate anything to help this girl. (Mora 1713-1720)

- So I think, my evaluation of myself is that because my judgmental side--I think is still there, but I think is toned down, you know, I could address it--I think ... that's allowing him, and accepting him, ... I would like to think that gives him the ability to flourish. . . Instead of um--because I'm sure even though you might not recognize it as judgments, I'm sure they hear it as judgments. (Leigh 630-748)

$\square$ That is the only way you can generate a different reality, is in the language you use. (Mora 1543-49)

$\square$.. oh and the part about sharing, Think about that, when you say it, it makes it more real. . . because if you're sharing anything, the changes that you've seen or the little, ah, the, ah unreasonable things that go on, ah, just by sharing it helps you, helps me anyway, relive it? And to revisit it and to, it rekindles the excitement . . . I can say it in my mind, but to verbally say it, just brings up everything it did that day. . . If you're sharing it with somebody else, it enlightens them, but it also helps me reaffirm, you know, what went on. You know what I mean?. . . Its more powerful than just, just the words. . . Its not that you're telling someone, its that you're experiencing it all over for yourself and allowing them the privilege of experiencing it with you. . Its just not something that falls on deaf ears, you know, people will walk away with it thinking about it and you'll, have it again." (Leigh 1208-41)

While only a small sample of the interview data available, these seven excerpts provide clear examples of how the metaphor cluster of "conversation as creation" 
manifests in the discourse and lived experiences of participating members of LEC's community. Notice how each interviewee expresses their personal understanding of the relationships between conversation/language, self, other, responsibility, power, reality, and possibility. Each excerpt suggests that for the LEC participants, creation is synonymous with the act of living, which for them, is the essence of conversation.

In contrast, another finding was that one of the interviewee's discourse revealed more "conduit" generated language than any of the others. This man noticeably (and admittedly) struggled to answer questions regarding language. He felt like he was not as aware of the power of language as he might be in his everyday speaking: "I don't measure my words, I guess" (Simon, 1554). His responses when discussing language tended to frame words as objects that could be grasped, had weight, could be tossed around, become projectiles and hit people (Reddy, 1993). Consider the following examples:

$\square$... commitment and integrity. . those words aren't, they're not tossed about lightly, and they carry great weight (Simon 1424-26)

- I'm like a loose cannon language-wise. And I say things that I have no idea how it may hit other people and for the most part, its just fine, and I don't have a problem. But I think that there are ah, times when, I could hurt people's feelings ... (Simon 1493-1504)

And its all, you relaying who you are to other people in, in, your language. (Simon 1495-96)

$\square$... those words aren't, they're not tossed about lightly (Simon 1424)

$\square$... almost like playing with nitroglycerin: when you use it, you mean it and, and you don't play with it, ah, nonchalantly, because the mean ... you're promising. (Simon 1425-7) 
Overall, Simon's discourse did display an awareness of the power of language to shape meaning and reality; however, his meta-language tended to display "conduit" framings for his conceptualization of how language carries meaning between people. His references to the location of meaning were mixed; at times he framed words as having the meaning in other instances he noted that words mean different things to different people. As Reddy (1993) notes, the "conduit" frame is so deeply embedded in the structure of our language about communication that "frame-conflict" such as this is actually expected. Of the other four interviews, three were with individuals whose backgrounds in philosophy and education (and two of which had participated fairly extensively in LEC) may have made LEC's metaphoric structuring more compatible with their pre-existing frameworks.

\section{Conversations as Structures}

The metaphors in the "conversation as creation" cluster just discussed structure the components of the process of creation and their relationships to one another. One of the products of communication as a creative act is reality, or more specifically, structures of various kinds and shapes for realities and "being" to occur within. The worlds human beings exist within, according to LEC, cannot be separated from language and conversation. Language and conversation in this instance are metaphorically mapped in very similar ways. Language can be thought of as the "totality" of words in which the possibility for various meanings and realities exist: a language "allows for" things to be. A particular vocabulary or way 
of speaking may allow for a reality that another does not. It is the use of language in conversation that "actualizes" and maintains a reality (Mulhall, 1996; Berger \&

Luckmann, 1966). In LEC, all structures for human existence and being are

products of conversation, sustained by conversation.

\section{LANGUAGE IS THE DOMAIN OF BEING}

Difference exists in language. (AC, 552)

$\square$ Nothing exists independent of language. (AC 11/95)

$\square$ Possibility exists in a vocabulary, in a way of speaking.

- There may be a reality that exists outside of language, but we have no access to it. (AC 586-87)

$\square$... what it is to be human has its own domain, and that domain is one of language--of communication--of conversation. (Comm. Prg. brochure, 1994)

$\square$ Language is the house of being. (AC 11/95)

Possibility lives in language. (AC 11/95)

$\square$ Our 'self lives in the language of others. (AC 11/95)

CONVERSATIONS ARE STRUCTURES (e.g. dwelling)

$\square$ You are born into a variety of conversations. $(\mathrm{AC}, 34)$

$\square$ The quality of your life is a function of the conversation you are dwelling in. (AC, 483-4)

$\square$ The frame for any breakdown lives in a conversation. (Brkdwn, p. 28, 19-20)

$\square$ This work lives in sharing, in conversation. (Brkdwn, p. 65, 20-21)

$\square$ The Landmark Forum is about seeing where the structures and ceilings that limit us are of our own making, rather than permanently in place. (SEF Brochure, 1996)

$\square$...we come to realize that the realities and circumstances of our lives really exist only as a product of the conversations that we are. (CAP brochure, 1996)

Language and conversation as dwelling suggest a particular type of structure-one in which something or someone lives, resides, exists. As stated earlier, all structures that impose a form on life/living are products of conversation in LEC. For example, a "world": "If we live in a world of conversation, we can invent a new 
conversation about something" (Brkdwn, p. 63, 31-40); or a "self": "Who I am is a conversation (someone speaking, someone listening)" (AC 14-16).

The "conversation as structure" metaphors allow for many different kinds of structures (and thus, many different types of conversations) that serve different kinds of purposes or simply have different dimensions. This fits with the idea that some conversations are more confining than others (e.g. a prison vs. an estate with many rooms and lovely grounds; a "racket" vs. "sharing"--see pp. 95-102; 121-126). Similarly, some structures are easier to enter and exit than others (a maze vs. a gazebo). There are some structures that are more enjoyable to be in, for example, a house with many windows vs. the windowless basement of an old university building. Within a structure's demarcations the space may be cluttered or roomy, orderly or in disarray. The important thing about these structures is to realize that human beings participate in the construction and maintenance of the structures that they exist within through their conversations. Thus, a structure may also be altered-or abandoned--through conversation. We can begin to see the coherence between the "conversation as creation" metaphors and the "conversations as structures" metaphors--the two sub-systems interact with one another to structure the processes and products of conversation.

Adopting the inference patterns of the "conversations as structures" metaphors would be likely to increase individuals' awareness of the consequentiality of their language and conversations for themselves and others. For example, if my partner 
persistently complains about something and I validate those conversations through my participation (e.g. agreeing or complying), I might become aware of myself as assisting in the maintenance of a constraining conversational structure. An interesting dilemma might arise between conversational partners if one operates from the paradigm of "conversation as created structure" and the other does not. Does one allow the other to persist in disempowering him or herself, much less, participate in that process by agreeing or listening passively? The following two interview excerpts hint at how operating from two different paradigms regarding conversation--in this case as it relates to complaining--might manifest in people's lives:

$\square$ for the last three years I've been their little complain-o-matic here, you know, listening, and just always trying to make them feel better but never helping them. And I've stopped listening to their complaining and I don't think they like me very much anymore. ... And after my seeing such revolutionary solutions to people's problems when they really wanted to do it, I try to say, "well, what about this," and try to use some of the help I found in the Forum to help them and say, "try to consider these other things." Well, nothing ever happened after two, three, four months. I just started saying things like, "It sure sounds to me like you don't want to help yourself," and they don't want to hear stuff like that. Instead of just listening passively and saying "oh you poor, poor poor poor whatever, I just started really listening for what was going on (Mora, 353-384)

$\square$ I'm just very willing to talk ... more openly with people and to confront them a little bit with their own--not confront them--but just ask them questions. . you know a lot of people like to gripe and stuff. I'll just ask them to explain themselves a little bit more. ... not that I have the answer or know what's going on, but I just, I'm a little more probing in conversations I guess ... especially when people are complaining. I've always thought that if you're complaining about something and you don't have the solution or you're talking to someone that can't possibly help you, then you're just bitching. . . But there 
are people who will just gripe and moan about anything. . . I'd just ask them, what's the solution to this problem? (Carl 552-572)

This sort of dilemma is also relevant to the notion of "integrity." If someone consistently fails to follow through on what they have said and I say nothing about this behavior (even thought I may secretly resent it), am I participating in the maintenance of a disempowering rickety structure for existence? What becomes important in adopting the "conversations as structures" inference patterns in this community is the individual's awareness of and responsibility for the integrity of his or her own conversational structures for existence.

Conversations as Structures: Interview Data. The notion of conversations and languages as "structures" or the basis for structures was evident in much of the interview data. Overall, the interviewees appeared to have an awareness of language and conversation as having power to hold or mold reality in various ways. Again, the notion of pattern and of careful observation must be kept in mind when reading through the following excerpts. While the individuality of one interviewee's expression, or even the significance of individual words, is important, it is when they are taken together as a whole that they provide a sense of how the "conversations as structures" metaphors are made meaningful by participants in the LEC community. In these excerpts are at least four observable patterns: (1) evidence of the interviewees' experience of conversational structures as having different sizes, shapes, and possibilities; (2) an awareness of how different processes of 
communication create and alter resulting structures, as well as move one into and out of structures; (3) an awareness of how structures created through language and conversation shape one's experience and reality; and (4) a sense of personal responsibility, choice and empowerment seen previously in the "conversation as creation" metaphor cluster.

$\square$ And, not living my life out of, what's right or wrong in the world, but living my life out of what am I committed to. (Ivan 1229-30)

$\square$ You want to be in the conversation. (Simon 1578)

I I mean, doctrine to me is similar to marriage vows ... when you give your vows, you create a structure in language to have your marriage in (Ivan 206465)

$\square$... its very important in the education process at Landmark. Its--you become aware that language is, is one of the incredible building blocks of relationships and, and of this world. (Simon 1617-20)

A And the most profound distinction that I got out of the Advanced course was that--the use of language. How reality is constructed for everyone and the-how people use language to generate your reality. ... that was very profound. (Mora 1133-37)

$\square$ well it seems like the distinctions in the Advanced course were working up to this distinction about language. . . it seems like everything um, the leader was trying to build up to this major distinction about how you create your own reality, you know, and its all just an artificial construct ... When that was finally--that distinction was finally made clear through her talking and through her language. . . They realized that the entire construct of their whole life was meaningless. Everything that they'd built up til now was absolutely worthless.

That the potential for what you can build for yourself was too frightening and too scary that the whole. . . It was too overwhelming. (Mora 1142-56; 1186-1204)

$\square$ "Decision" means you--that you killed [the other options] off and you have only this one left. ... "choice" means you still have all the other options. You know, so every choice that you make doesn't narrow your life down or limit your 
possibilities at all. . . Just paying attention to the language that you use. (Mora 1236-51)

whereas instead of communicating from everything always being about you and things being so personal to being able to discuss something outside of that reality where its always about you .... really get beyond that and really look at the topic. When I've been able to do that--at times which is so much freedom and so many possibilities of how things could go. Just so--its so much more open and such an opportunity for people to really communicate with each other, you know, really hear each other--I've really heard some people. (Jenna 816-832)

Evident in the excerpts above is interviewees' awareness of the consequentiality of their conversational behavior, both in terms of the structural conversational products created and the impact of those structures on their immediate and future experiences, reality, and communicative behavior. Another recurrent theme, especially in the last two excerpts, is that of "possibility." Recall that whole of LEC's curriculum is often referred to as "a conversation for possibility" (AC jotted notes), a label which brings together the process and product frames for conversation. That is, an LEC course in a process that creates possibility by creating conversational structures that allow for and support possibility. The interdependence of the "conversation as creation" and "conversations as structures" sub-systems is evident in the above excerpts, as interviewees simultaneously comment on the process, as well as product, of their conversations.

\section{Integrity: The Alignment of Process and Product}

The idea that conversation is and creates a structure entails the notion of "integrity": the wholeness, completeness, and soundness--the "structural integrity"-- 
of the structures we create to exist within. Integrity is created through an "alignment" of one's words/speaking and the actions that follow from speaking (see pp. 110-113 for a review LEC's version of "integrity"). LEC's notion of "integrity" is conceptualized as "alignment" or "no distance" between words and action, between what one says and what one does.

Being "out of integrity" is conceptualized as "a mess" that requires "cleaning up," which could be related to a conversational structure through the cliche about "not having your house in order." Consistently failing to "be one's word" is said to "damage" the listening one has to "show up in"; that is, the conversational structure in such an instance lacks "structural integrity" and may provide a constraining or undesirable form for one's experience and self-expression. Of course, integrity is a way of being, and all ways of being in LEC are created through conversation, which means integrity is a conversational structure (more on this in next section). The idea that being out of integrity damages listening includes how one listens to one's self. For example, consistently telling myself "I will get up early tomorrow and exercise" but consistently failing to follow through with the promised action may result in a weak personal relationship with my word. In the future when I speak about future actions, I may listen to myself through past experience and not really intend to follow through. The significance of listening in the concept of integrity is entailed in the idea that "what gives speaking power is listening" (AC notes). 
INTEGRITY IS ALIGNMENT BETWEEN WORDS AND ACTION

INTEGRITY IS BEING YOUR WORD

\section{BEING OUT OF INTEGRITY IS A MESS}

$\square$ Ultimately integrity is honoring our word as Self; Alignment of network of conversations that you are . . doing what said or saying we didn't $\&$ cleaning it up. (FIA 3137-42)

$\square$... an opportunity exists to align conversations to empower who you say you are and how you intend to live. (FIA, 3146-48)

$\square$ Integrity . . alignment of conversations with who I say I am (FIA, 3102-04)

Integrity: two tongues, one in mouth, one in shoe; what you say and what you do; Only two options: Say X, did X, Say X, did Y--acknowledge, clean it up, then do what said (AC 165-69)

$\square$ When you start practicing your word as your self, amazing things will happen, often at the level of miraculous." (Brkdwn, p. 11, 6-8)

$\square$ Not following through on commitments/promises damages our listening for our self and other's listening for us. (Brkdwn p. 11, 9-13)

T The key is to make a commitment and be your word--not just lips moving for the sake of talking ( $\mathrm{AC}$ 626-27)

The meaning structures that frame integrity as alignment between the process and product of speaking focus attention on the relationships between speaking, listening, actions and outcomes. This alignment is conceptualized as a requirement for actualizing the potential power of conversation: "Without integrity, nothing works; without integrity, no power" (Brkdwn, p. 10, 41).

Integrity: Interview data. The concept of "integrity" also emerged in the data. Three interviewees reported experiencing "integrity" as a concept of significant personal importance. The concept of integrity was metaphorically framed as providing alignment between the processes of "conversation as creation" and the products of "conversations as structures." Integrity was also framed as the "structural integrity" of those resulting structures we exist within as human beings in 
"a world of conversation." Interviewees' seemed to focus primarily on the aspect of "integrity" that relates to the alignment between processes and product; that is, the significance that "being one's word" has for an individual's speaking and related actions. That participants would focus on the process of integrity, rather than the more static notion of the "structural integrity" of any one conversational structure seems a natural extension of LEC's worldview that frames life as an ongoing process, constantly in motion just as human beings are constantly making meaning through conversation.

$\square$ What I shared was--I got up and said that its unbelievably profound and I have to very careful about what I say in the future, if I'm going to have any integrity at all.(Mora, 1184-1204)

-... what you speak is what you are, and that's where integrity comes, you live what you say or you don't, you either have it or you don't. It was so simple and so profound .... you either have it or you don't, I guess, and its something that you really want to have. (Mora 585-627)

$\square$ Richard was talking about integrity, distinguishing integrity as honoring yourself as your word, that when you saying something, having integrity is being what you say. If you say you're going to be some place at four o'clock, you're a human being that is being: arranging their life in such a way that they are at that street corner at four o'clock where they said they were going to be meeting so and so. To being--you know, when you say "I do" at an alter, you just gave your word there, being "I do." (Ivan 489-501)

(a) You know, part of having integrity is cleaning up the messes that you've made when you haven't kept your word. So I could see that I had a mess with my father to clean up. ... and I said, "Well, I'm going to go call him," and he said "Ivan, where is the integrity gonna live?" . . . And then I said, . . " That integrity is going to live for me when I actually pick up the phone, and dial the number and have the conversation with him. "Its not going to be me just having this epiphany realization that, I had not kept my word and, it really sucked. . . . But that in the actual act of picking--in the real world--of picking up the phone, 
having a conversation with my dad and cleaning it up. So that was really powerful experience. (Ivan 546-561)

The "conversation as creation" metaphor finds some overlap here. For example, in the last excerpt Ivan says that "integrity" is going "to live" for him "in the act" of picking up the phone and having the conversation. That could suggest either that the act gives life to integrity or that integrity will live "in" the conversational structure. Since conversation is both process and product, this kind of overlap is understandable. Clearly, "integrity" occurs for these participants as a way of being created in an alignment of their words and actions and includes an awareness of the consequentiality of their speaking. These excerpts reveal that the notion of integrity is a meaning structure that has interviewees experience the interdependence of conversation and ways of being.

\section{The"Being" in Human "Being"}

\section{The "Design Principles" of Human "Being"}

LEC refers to the totality of their distinctions as "the design principles of human being." Not $a$ human being, but human being (a typically passive noun, reads as an active verb). This emphasis makes sense when you recall that LEC's work is ontological in its focus. To say that human being has "design principles" definitely imposes a structure on an inherently abstract and somewhat mysterious aspect of human existence. Recall that LEC also calls their work a "technology" which, in combination with "design principles of human being," suggests that "being" is an 
empirical phenomenon that can be understood and manipulated via the methods and materials of an applied science. A design can be defined as "the order or arrangement of the components and details of something in accordance with a plan; a well-thought out purpose: intention; layout, plan; blueprint, schema" and a principle "a fundamental truth, law, or postulate; a rule or code of behavior; an underlying or established rule or policy; a scientific law underlying the working of natural phenomena or mechanical processes" (Webster's' II, 1984, p. 192; 556).

These definitions imply that human "being" 1 ) is composed of aspects that are strategically organized and can be systematically described; 2) functions according to scientific laws or underlying rules in observable ways; and 3) is either a naturally occurring phenomenon or mechanical process or perhaps both. At the very least it is suggested that "being" has a particular layout, a topological form or organization about which basic claims can be made.

This discussion of "being" corresponds with the root-metaphor HUMAN BEINGS ARE MEANING-MAKING MACHINES. This structural metaphor highlights the aspect of being that is designed to perform a particular task: meaningmaking. As meaning-making machines, human beings are framed as "hardwired" to make meaning, as an inevitable part of human functioning. At first glance this position would seem to contradict LEC's primarily existential position regarding human existence. That is, if "existence precedes essence," then how is it that human beings possess this predetermined function (Palmer, 1995)? The difference, I 
believe, is that LEC does not claim that human beings behavior is predetermined, but that part of the "design" of being is organized to function in a particular way, to perform a particular task, just as an internal organ is designed with a particular function. While human beings share a form that gives shape to their existence, within that form, any number of unique expression within that form are possible. One of the analogies LEC has used to illustrate this idea is that of the sonnet. While a sonnet requires a rather rigid form, within that form there is total freedom to create.

As discussed earlier, we cannot not make meaning--that aspect is automatic-but we can control or choose the meaning we make. A potential entailment of a machine metaphor is that machines can be automatically or manually operated. Automatic Being: "Rackets" and "Winning Formulas"

"Automatic being" is an already-determined way of being in the form of a reaction. LEC calls these reactive ways of being "thrown." "Thrown" reactions are "triggered" by meaning structures (conversations) that typically go undistinguished in the moment of reacting, at least initially. That is, an automatic way of being and the "conversation" that triggers it do not naturally occur or exist for the individual in the moment of reaction, unless of course, they are distinguished. It is the meaning we make of things--a conversation--that "gives" or determines our being. If the meaning-making machine is running unsupervised (undistinguished) on automatic, the meaning produced and the being that follows will be produced from what is automatically there. What is automatically and effortlessly there, according to LEC, 
are inherited meaning structures and ways of being, such as standards, ideals, expectations, "rackets" and "winning formulas."

The distinctions of "racket" and "winning formula" help to illustrate the three structural implications of LEC's "design principles" for our understanding of human "being": 1) that being is composed of aspects that are strategically organized and can be systematically described; 2) that being functions according to scientific laws or underlying rules in observable ways; and 3 ) that being is a collection of naturally occurring phenomenon and/or mechanical processes. LEC has articulated a form-created a structure--for each of these ways of being that makes what is typically automatic and invisible, recognizable and therefore, accessible.

And I think, I think like, tracing the roots of it, I think they've been able to target, what it is and how it works in people's lives; being able to distinguish that we have ways of being, in life, that look like this. And then they created a structure for that way of being called "racket" and a structure for that way of being called "winning formula." (Ivan 2250-65)

"Racket" and "winning formula," in addition to being "design principles," are also structural metaphors. Unlike conventional structural metaphors, the experiential domains for these two metaphors had to be compiled for participants by LEC during the Forum and then labeled "racket" or "winning formula." Having discussed these two distinctions in some detail earlier (see pp. 95-102), I simply want to focus on the way each of these offers a clearly organized set of functions that provide access to recognizing two automatic ways of being and potentially, choosing alternative ways of being. 
The automatic mechanism called "racket" has a very clear structure that allows an individual to quickly identify whether or not he or she is "running a racket" and if so, to evaluate the costs and payoffs in an orderly, economic fashion.

A Racket: a persistent (or fixed) way of being (doing or having) plus a complaint.

[what is my way of being?]

Payoffs: dominate/avoid domination; be right/make someone or something else wrong; justify self/invalidate others.

[what does this way of being get me?]

Costs: life; love; self-expression; health; vitality; relationship; accomplishment, etc.

[what does this way of being cost me?]

The mechanism's central function in this case is to deflect personal responsibility, a function suggested by one of the definitions of the word "racket": a fraudulent business (Webster's II, 1984). The entailment of "fraudulence" suggests that the "racket" is a cover or "front" that distracts attention from the "real" goings on "behind the scenes."

Although "winning formula" does not have the clear economic structure found in "racket," $\mathrm{a}$ "winning formula" can easily be identified through self-reflection and assessment: what is it one does to succeed in life--what are the practices and ways of being that one repeatedly engages in as a "formula" for success? This "formula" can most easily be identified by considering how one responds to situations of urgency or crisis. Recall that a "winning formula" is a way of being generated to

\footnotetext{
${ }^{8}$ Although the interpretation can be drawn out when one considers that business tends to apply such scientific formulation to successfully compete in the marketplace.
} 
avoid and compensate for a past "failure-to-be" experience. The notion of

"compensation" invokes another familiar meaning related to economic exchange.

These two examples illustrate the kind of systematic, observable, rule-governed structures that follow from the metaphor of "design principles." One interviewee who had just recently completed the Forum commented that she found the "racket" structure to be particularly useful to her:

Well, what's been really helpful was to really recognize a racket when its going on. . . so what helps me is to go: okay, what am I complaining about here? Okay, and then, What am I feeling? What am I being when I complain about that? That helps me to identify . . that it is even a racket. Okay, so, the way that you are when you have a persistent complaint, that that's been coming up and coming up, more than a couple times, and then to recognize what the payoff is. . . then, to examine what the cost is, has been really helpful for me. And to recognize that it is either costing me intimacy and love, or my happiness, or self-expression. . . but to be able to work that through in a method, that's internal of my head ... I I have the ability, if I take the time, to work that through, and therefore not lay it on someone else, and then take the responsibility for myself. . . And what this does, is it gives me a channel to take it--and I envision it like a huge funnel, where, here I have this huge amount of feeling and, and all this jumbled stuff that's being fed into a funnel, and recognizing that its a racket or not a racket, is just funneling it down to exactly what the cost is. . . (Leigh 476-540)

For Leigh, the distinction "racket" seems to provide a useful method or tool that when applied, creates clarity for her. The economic structure also seemed to provide illumination for Leigh, focusing her attention on the consequentiality of her "being" and behavior, and providing the possibility of being responsible. The "design principles" called "racket" and "winning formula" are automatic ways of being that 
human beings inherit--part of "machinery" implied by the root-metaphor "meaningmaking machines."

\section{Automatic Being is a Thrown Switch}

Building on the machine metaphor, "automatic being" can be framed as "thrown switch." The "thrown switch" structures the aspect of reaction, suggesting the experience of force. It also entails that the switch is a preprogrammed (in the past) mechanism.

\section{AUTOMATIC BEING IS A THROWN SWITCH}

$\square$ JT ask how many of us are thrown when we find out we've been doing it "wrong," when we thought we were doing it "right." (Brkdwn, p. 45, 22-23)

$\square$ In the right circle she writes the words: wants, reasons, explanation, justification. This is the world of mechanical, automatic--where an incident triggers this behavior. (Brkdwn, p. 55, 22-24)

$\square$... look at our standards and ideals--not in order to change or get rid of them, but to "unconceal" them in order to look at how much of our automatic behavior comes out of "being run by" them. (Brkdwn, p. 27, 31-34)

口 "thrown" position (different from taking one)--taken from past (FIA, 3350-51)

$\square$... automatic behavior comes out of being run by our standards and ideals-can't be present to what's so or engage in effective action while being used by them. (Brkdwn, p. 27, 40-42)

$\square$ Force--Throwness (Already Always) (FIA, line 3096)

$\square$ Rackets and Winning Formulas generate meanings, but they are mechanisms. (AC notes, 11/95)

Notice that when "being" is automatic, the individual is framed as being "run by" the past-based meanings, which highlights the aspect of giving up control. When a "thrown" way of being remains undistinguished, giving up control is typically experienced as not having control. The experience of not having control is also 
congruent with the earlier mentioned premise that automatic ways of being defer personal responsibility.

The orientational metaphors operating in the metaphor of "automatic being" are also clear: AUTOMATIC BEING IS NOT BEING PRESENT (or in the past) AND AUTOMATIC BEING IS A FIXED POSITION. Interestingly, automatic being is also coherent with the orientation of "being stopped." This might be explained by considering that when the switch is thrown, we stop, and the machine takes over. "Being stopped" and "automatic being" are also congruent with LEC's notion of a "breakdown." A "breakdown happens when we are committed to something and get stopped" (Brkdwn notes). Before being distinguished as a "breakdown" however, the experience of "being stopped" ("thrown") tends to occur as what LEC calls "an upset." "An upset" is an emotional reaction to a situation that "runs" an individual. It is automatic, not present, gives away responsibility, has drama attached to it, and is "thrown" (pilot log). A "breakdown" is rather, "something we can be powerful in the face of" (Brkdwn, p. 38, 21-22); a breakdown is something to be handled, which entails having the appropriate tools to "resolve" the breakdown.

While machines can be complex, they can also be understood and controlled if one has the appropriate knowledge, skills or training (enter LEC!). In this way, the structure of the machine metaphor suggests that being and behavior are accessible and controllable. The power to operate and control the machinery is located within the individual (although sometimes handling a breakdown consists of asking for 
"coaching"). This metaphor distracts our attention from the immense difficulty in maintaining the kind of awareness and intentionality that this kind of "manual being" would seem to require, since, according to LEC: "We are either (1) generating possibility or (2) being used by what we inherited" (AC, 11/95). Consider another aspect that is down played by this metaphor: Would not mastering LEC's technology make it a past-based system of meanings that could give our being automatically? If one is being automatically "run" by LEC-derived ways of being, isn't that still being "run?" Of course, LEC would not claim that automatic meaning and being were "bad" (just possible ways to be), but the value implications between controlling or being controlled become obscured when one moves to consider LEC as the source of past-based meanings for automatic being.

\section{Being is a Conversation}

Focusing on "conversation" as the process and product of meaning-making is what provides access to controlling or directing meaning-making, and thus being. The idea that "being is a conversation" has been explored in the worldview explication as well as in the section on "conversation" metaphors. Being is constituted and expressed in conversation; that is, conversations give rise to ways of being and a way of being gives rise to certain conversations that may occur at the intrapersonal, interpersonal, family, group, public and/or global levels. This relationship between human "being" and conversation is another example of what LEC's calls the "design principles" of human "being." Mapping the experiential 
domain of "conversation" onto that of "being" gives "being" a recognizable and accessible form. The dimensions of conversation--such as language, speaking, listening, participating or not participating, topics and changing topics, kinds of conversations, beginnings and endings--become mapped onto "being" and provide a potentially new understanding of our own and others behavior, as well as new possibilities for future action. The access provided by this mapping also suggests a locus of power in the individual regarding "being." If who and how I am being is a product of my conversations, then those conversations are also my access to consciously choosing and enacting my ways of being.

While human beings have the power to distinguish the conversations giving them being at any given moment, for the most part they go through their days without doing so. The key to empowering one's self in the domain of "being" is being able to distinguish, and then choose, the conversations that one is being from . The relationship between "being" and "conversation" can also be conceptualized as a figure-ground relationship. "Being" occurs as the figure rising from a particular conversational ground or context. Distinguishing focuses one's attention on the "conversational ground," providing the opportunity to alter or shift this context which results in a different way of being or figure. One aspect of LEC's "technology" that addresses this is the distinction between "what happened" and "interpretation" (see pp.91-92). Distinguishing between "what happened" and the meaning one made about "what happened" brings one "present" which reveals the 
"conversation" and the resultant way of being, thus providing the opportunity for choice. Recall that "power is a function of distinguishing--the more you can distinguish, the more access to power you have" (Brkdwn, p. 22, 30-31).

Distinguishing is necessary for altering an automatic, ineffective, or undesirable way of being, but it is not enough: "We can distinguish it, but that doesn't necessarily stop it" (Brkdwn, p. 50, 36-37). Shifting from an automatic/ineffective way of being requires at least three things: (1) "getting present" by distinguishing the conversations giving one being--"transformation begins with being present to what's so" (Brkdwn, p. 8, line 40); (2) choosing whether to continue with the current way of being or to generate a new conversation to "be" from; and (3) generating a new conversation, if that is what one has chosen to do (e.g. one can choose to continue running a "racket"--I have!) "Its about distinguishing conversations as conversations, and in that moment, having the freedom to be present to what is, and to be present to what's not, and to make a choice" (Ivan 2792-96).

\section{BEING IS A CONVERSATION}

口 Ask yourself the question: "What's informing your about to be?" Allows you to make a distinction so you can get present. (Brkdwn, p. 38, 6-7)

$\square$ If we live in a world of conversation, we can invent a new conversation about something. (Brkdwn, p. 63, 31-40)

$\square$ Being who you say yourself to be, rather than automatic, thrown (Brkdwn, p. $55,14-15)$

$\square$ you being cause for another being cause is a conversation called "enrollment" (AC, 194-95)

- Maybe have to give up there is anything out there except what you speak-world as a perfect correlate to who you are being. (SELP, 271-72)

$\square$ what reality is one endorsing through behavior and speaking--what reality are you being? (Brkdwn, p. 27, line 18-20) 
The metaphor BEING IS A CONVERSATION is consistent with the metaphor CONVERSATIONS ARE STRUCTURES. Recall that human "being" occurs within "structures" created through conversation. This consistency can be depicted in this case in the form of a deductive syllogism:

Conversations are structures.

Being is a structure.

Therefore, being is a conversation.

This degree of consistency between metaphors, according to Lakoff and Johnson (1980), is fairly rare. Consider for a moment that LEC's worldview/metaphoric system is: (1) intentionally and artfully manufactured; (2) occurs as new to most participants; and (3) presented as a rhetorical argument that must be made plausible and accessible to participants. In light of these considerations, one can understand this extreme degree of consistency. Shifting the frame slightly to specifically focus on "being" as a conversation provides an emphasis particularly relevant to LEC's "applied ontological" material and more closely tied to interests of participants seeking an access for an empowered existence. The influence of the root-metaphor LIFE/LIVING IS A CONVERSATION shows up strongly in the subsystem "being is a conversation." Human "being" is essentially inseparable from the concept of living, since "being" is the form life takes for us. "Being is a conversation" is a more specific subordinate mapping within the superordinate mapping LIFE IS A CONVERSATION (Lakoff, 1993). 
Automatic being $\&$ being is a conversation: Interview data. The interview data presented here provides illustrations of how the metaphoric structures in the domain of "being" (including the two major divisions in this domain--automatic being and "being is a conversation") manifest in participants' discourse. The themes operating in this metaphoric subsystem and evident in the interview excerpts include: (1) the experience of automatic or "thrown" being (including rackets and winning formulas); (2) the LEC distinction between "what happened" and "interpretation"; (3) the idea that "being is a conversation"; and (4) the role of distinguishing in gaining access to and power with conversations. Also detectable in the following sample of interview data is the influence of the inference patterns of all three rootmetaphors: LIFE IS EMPTY AND MEANINGLESS, HUMAN BEINGS ARE MEANING-MAKING MACHINES, and LIFE/LIVING IS A CONVERSATION.

The first three excerpts provide especially clear examples of automatic being, including the concepts of "being run" by modes of automatic being (e.g. "rackets); that automatic being is a "reaction," which LEC frames as a "thrown switch"; that modes of automatic being function as "mechanisms" and that such mechanisms deflect personal responsibility; and that "automatic being is a fixed position" or entails "being stopped." Also apparent in these excerpts is that "being," which includes automatic being, is a conversation that can be distinguished and subjected to the power of choice. 
$\square$ This whole either or conversation that I have for me, its just a total, its a way of having me not be responsible for sharing Christ with people. Or sharing the Forum--its a way of not having me share. . . Because I'm stopped. . . when you use language, its a racket. And when I'm running that racket, either or, one's right, one's wrong, and I'm not sharing anything. . . in the moment of being stuck, I'm not sharing anything. And sharing is more about freedom. Its like, I can share Landmark or my faith, freely. . . . I can just so see that, that that's a mechanism that has me simply not share. You know, when I'm sharing, I'm just sharing. You know, its like, it is just sharing. (short laugh) There's no right or wrong about it. (Ivan 2815-37)

- ... the other big one was when he got to the point about, you're just a robot on automatic. That really got to me because, um, I saw that I really was. I saw that I had a really strong visualization about getting approval from someone ... and as soon as I got it, I needed it again. And as soon as I got it, I needed it again. It was like, I was never there, I was never just going to be, yes! I'm okay. Because as soon as I got it I needed it again. . . . . . That's just who I am. That's just what I do. And I am a robot. I mean like, I am just that mechanism. . I was like, great, this is really wonderful. I'm a total robot, I really see that and now I've gotta live with it?. . . That you know, no matter what, I was always going to need approval and it was so just mechanical and if somebody had said, you're mechanical to me I would've argued them to the ground. And then when they said . . life is empty and meaningless, it was like, oh, okay, well that doesn't mean anything that I do that and I'm mechanical, but if I can see that I'm that way then I could actually choose something else--that's when they talk about choice (Jenna 525-68)

$\square$... how you speak to people and the way that you hear people. . . the role that you're playing . . . your the setup that you've got created for yourself and the way you automatically react to the world around. . you can observe that happening in yourself if you're aware all the time . . . you can catch your automatic reaction and you can say, "hey that's an automatic reaction, what I-what am I doing? And you can see those automatic reactions in other people . And you can catch yourself doing that ... and try to not do it. You know, its automatic, your automatic responses. So you can get a different conversation going on, instead of automatically responding, you take control of your life and respond in a way that's, you know, more reasonable to what you're trying to do. (Mora 284-315) 
The next five excerpts highlight the ways in which distinguishing and distinctions give access to conversations and disempowering ways of being, and thus, provide the possibility of creating more empowering conversations and ways of being. The first four excerpts point to the LEC distinction between "what happened" and our "interpretation" or "what happened." Another distinction mentioned in the interviewees' discourse is simply "conversation," which seems to provide Leigh with empowering access to her intrapersonal communication.

$\square$ You know, its like, having a great wonderful, intense, special, whatever kind of a life, those are all interpretations as well." . . . [what happened/interp. model] its not something to be take completely literally. That's its just $a$ really powerful structure that you can live your life out of that brings sheer possibility to your life." . . The metaphor of interpretation and what happens, that--saying, "you never listen to me and you're a jerk and I can't stand you" is quote unquote "just an interpretation," but . . . "a really powerful life of beauty and passion". . . . That's still an interpretation as well. its just--its just distinguishing that you're living your life from a particular place. ... And when you can see what they are, you then have choice in the matter. ... You can then choose, so what interpretation are you going to choose? (Ivan 402-36)

$\square$... understand that people aren't out to do something nasty to you, they're just being themselves, and they might do something that you interpret, but to look at what--actually just look at what they--look objectively at what they did, not why they did it to you, which is your idea of what they've done. But just to look at what actually happened. And then just say, you know, then any grudges or problems you've had with anybody just schwew--they seem to slide away. (Carl 411-419)

$\square$ Being a human being is being a conversation that is not distinguished as a conversation ... . So, for example, when that woman came home and her husband said that, she said "you don't love me, I, you know, you hate me, never want to talk to me," she was being something in that moment. She was being the conversation "he doesn't love me," and maybe even "I'm not very loveable," and "this relationship sucks," she was being that. But it was a conversation that she was having, that she was being, and she did not distinguish it just, as, a 
conversation. . . But as real for her. . . And in that moment, she was being that. (Ivan, 442-453)

$\square$ I used to catch myself that I would make these, what I called assumptions, not knowing they were really the conversations that I was having to keep myself, either justified or righteous or you know, and so its really freeing to know that, that it is a conversation that I'm having with myself, you know, and that it really is destructive. It keeps me from having what I really want. Its not beneficial to me. So, when I say conversation, its this internal voice, that's almost, not my friend, you know. It keeps me from really, having the best of what I could have. The self-talk that goes on, where nobody sees it or hears it, that talk you out of everything. . . . And so its, been very empowering, [to determine] what was really said, or what really happened. But to call it, you know, something, is really helpful for me. Um, it stops the spinning. . . I guess it can be empowering as well as disempowering, the conversations? (Leigh 9751036)

D ... and something else that was said in the Forum is that "life is a conversation." And when you can distinguish the conversations that you have, the automatic ones that come from, lets say your past, or comes from something that happened, you know, yesterday or the day before or when you were three years old--when you can distinguish that were started then, as conversations, you don't have to automatically be run by them. You don't have to take them as, the quote unquote, "real" thing. . . . You then have the power, to choose another conversation to have about the matter. (Ivan 459-471)

These excerpts suggest that the metaphoric meaning structures regarding automatic being and being as a conversation have found some meaningful expression in the interviewees' understanding or their own and others' behavior. Also evident in interviewees' discourse is a sense of empowerment and motives for action traceable to the metaphors in this sub-system and to the interaction of this sub-system with the root-metaphors and other metaphoric sub-systems.

\section{Transformation}

The transformation that LEC intends to create seems to occur as a paradigm- 
shift: an alteration or modification of one's worldview. Consider that a "paradigm" of any kind is composed of an individual's or a culture's accumulated knowledge about something. What we know is primarily a function of past experiences and various kinds and conclusions about those experiences. When those past experiences are used automatically as the basis, foundation, or structure for interpretations and actions in the present, they may represent a limitation. Especially when this already existing reference point or structure is not questioned or open for questioning, but taken as "Real," "Right" and/or "True." Landmark recognizes this as a "design principle" of human "being," that is, human beings will always use what they know and understand from the past to interpret and understand things in the present. The point is not to cease this practice, but to become aware of it, and thus, gain the power of choice.

The specific parameters of the "paradigm-shift (outcome, shape, appearance, magnitude, direction) are left undefined by LEC, allowing them to be created by the individual, thereby accommodating the individual needs and goals of those seeking "transformation." Thus, LEC broadly frames the paradigm participants are shifting from as a structure, substance or position comprised of "what we know" (or think we know) about the world (relationships, ourselves, careers, etc.). Knowing and knowledge are not conceptualized as "bad" or limiting in and of themselves. The constraints are self-imposed when human beings adhere, consciously or not, to "what we know" as "the way it is." LEC's framing of "what we know" is closely 
tied to one of this community's key concepts: "already-always." "Already-always" represents ways of knowing, listening, interpreting, etc., that are derived from one's past experiences and one's culture, and that tend to function much like conventional metaphors are said to function in our conceptual system: mostly unconsciously, automatically, and without noticeable effort (Lakoff, 1993). Transformation, then, involves shifting one's relationship to a domain of knowing and/or knowledge, so that "what we think we know" about something becomes "one possible way."

The metaphors in the domains of "knowing" and "transformation" correspond roughly to the root-metaphor LIFE IS EMPTY AND MEANINGLESS. Part of the shift to consider life as empty and meaningless includes seeing that knowledge and the structures arising from it are not inherently meaningful or true or valuable: "I don't know' is only available from empty and meaningless" (CAP, jotted notes). Seeing life from "empty and meaningless" allows for the realization that "the way we see it isn't necessarily the way it is" (LEC Internet Homepage). The other crucial correspondence is that of "conversation." Recall that "conversation" creates structures and those structures can be confining or freeing. "Knowing" is a particular kind of conversation about something that can create a constraining structure and a resultant way of being that could be called "stuck" or "fixed" (more on these orientations in the next section). A "knowing" conversation might occur like certainty, rigidity, or simply basing our actions on assumptions; all of these create limitations of one kind or another. 
Becoming aware of the conversation as a conversation (which you may recall involves a process of distinguishing): (1) provides the possibility of altering the structure, (2) giving up a position, and (3) inventing a new conversation "not given by the past" which can create a "shift in being."

Transformation in LEC occurs in relationship to something or someone, such as a specific relationship; a physical position, direction or destination, a particular topic like money and so forth. Transformation is a process that involves a point from and a point $t o$. For example, the transformation of a creature from a caterpillar into a butterfly alters that creature's form, mode of transportation, and the way in which other creatures interact with it.

\section{Transformation From Structures of Knowing}

In this first set of metaphors the from point of transformation is metaphorically framed as a constraining structure that imposes confinement. Recall from previous discussions that in LEC, CONVERSATIONS ARE STRUCTURES and that some structures are constraining while others are not.

\section{WHAT WE KNOW IS A CONSTRAINING STRUCTURE KNOWING IS CONFINEMENT}

$\square$ Our shoulds and shouldn'ts are so much a part of the way life is for us, that we often end up living within the boundaries they establish without appreciating the all-pervasive impact they have on our lives. (Sharing, par. 47)

$\square$... we have restricted ourselves to being and acting inside a world that is narrowed to and defined by these options. (Sharing, par.48)

$\square$... it is our past experience that ... sets the limits of what can and cannot be . the result is a future that is some variation or improvement on what has been.

口 Transformation and possibility in being don't live in figuring it out [knowing]. (Brkdwn, p. 49, lines 34-35) 
$\square$ We need freedom from already-always structures about appreciation and acknowledgment. (FIA, 3437-38)

WHAT WE KNOW IS A SUBSTANCE; KNOWING IS A FILLED CONTAINER $\square$ The listening we are is filled w/what we know--no room in the cup. (AC 49-50)

$\square$ The more you know who you are, the less possibility--based in the past. (AC 38-40)

$\square$...we often relate to each other as if we already know ... this kind of alreadydetermined way of being ... leaves very little room to see newly. (Sharing. par.56)

WHAT WE KNOW IS A CONSTRAINING STRUCTURE is an example of an ontological metaphor which imposes boundedness on the abstract phenomenon of "knowledge." The second mapping, KNOWING IS CONFINEMENT, is a structural metaphor that maps the experiential domain of "confinement" onto the ontological condition of "knowing." These metaphors suggests that what humans believe they know determines what is thinkable, doable, and overall, what is possible for them. The notion that "knowing is constraining structure" builds on conventional metaphors such as THEORIES ARE BUILDINGS (Lakoff \& Johnson, 1980).

LEC's metaphor, however, suggests that remaining within a structure of "knowing" is a self-imposed and often invisible "prison." In other words, that we are confined does not occur, and thus, the "confining structure" does not exist: it is an undistinguished part of the background. Congruent with the ontological focus of LEC, what makes a structure of knowing constraining is who one is "being" about it: being that what you know is the way it is vs. being that what you know is $a$ way it is. 
The third metaphor, KNOWING IS A FILLED CONTAINER, is also ontological, building on such conventional mappings as THE MIND IS CONTAINER (e.g. "My head is full of ideas"). Unlike the conventional MIND IS A CONTAINER mapping, LEC's metaphor is not referencing just one's mind, but the whole of one's experience. That is, "what we know" constrains or limits what is thinkable as well as what is doable. Recall that "life is empty and meaningless" and that emptiness in LEC is possibility. A filled container does not allow for possibility. Framing "knowing" as a "filled container" is coherent with the previous metaphor of the constraining structure, as a filled container also imposes constraints. The metaphor suggests that "knowing" leaves little space in the container for possibility, which by definition in LEC is nothing; creating newly can only be done from nothing.

The previous section focused on the point from which transformation begins, a place where being and structure come together. The next mapping structures the process or experience of transformation as a breaking through the constraining structure and gaining freedom from the confines of "knowing."

\section{TRANSFORMATION IS BREAKING THROUGH WHAT WE KNOW} TRANSFORMATION IS FREEDOM FROM KNOWING

$\square$ You break through the confines of even the best conventional thinking, opinion and theory. (Forum brochure, lines 2705-9)

- The Landmark Forum provides an opportunity for a breakthrough. (Sharing, par. 31)

- To risk what we already know or have for what is possible; to step outside of habitual ways of acting and thinking. . . (Sharing, par. 19)

- The Landmark Forum offers another view--it proposes that we can stand 
outside the already existing options, outside what we already have or know. Standing there, standing in what is possible, the world that becomes available to us is. . a world where we have new freedom and power to say who we are and what our lives will be about. (Sharing, par. 60)

$\square$ The Landmark Forum is an invitation to move beyond the limits you have set for yourself, the constraints you have imposed on your own life, breaking through to new levels of performance and ability." (Forum brochure 2662-65)

"Breakthrough" is a key concept in LEC. A "breakthrough" is what someone experiences when they suddenly see something about themselves that they had not previously realized. The experience of "breakthrough" provides something by what it reveals. The experience of a breakthrough, or "transformation," is first, becoming aware of the structure or structures that confine us for what they are. The ability to distinguish one's framework as a framework and not "the way it is" opens up the possibility of seeing the world in a variety of different ways: "transformation is looking to create a relationship to life that isn't 'given'" (Brkdwn, p. 46, lines 6-7), one that is "open to being invented in any number of ways." Transformation here is framed as leaving or moving beyond a confining structure of "knowing." This metaphor is also coherent with the "filled container" metaphor, since leaving a filled container could also be framed as "moving beyond" and "stepping out of."

Confinement is conceptualized as self-imposed--"the constraints you have imposed on your own life"--thus, the power to alter those boundaries is located within the individual.

Transformation then, is simultaneously a process and a singular moment. Consider the analogy of the butterfly. While time is an element in its transformation, 
there is also a singular moment when it "breaks through" its cocoon and to the observer there is no longer a question of its nature: it is a butterfly. Consider another previously mentioned analogy of the structure of a prison. There are two primary ways to leave a prison in order to be someplace new: to be set free or to "break out." There is some "in-between" time involved in whatever planning or procedures that must take place, but there comes a moment in that process when one is no longer physically in prison. Once free of the structure of cocoon or the prison, the "transformed" entity no longer interacts with the world in the same ways.

\section{Transformation is "breaking through": Interview data. The following excerpts} from interviews provide illustrations of interviewees' experience of transformation as a moment of breakthrough while also highlighting the possibilities available "on the other side" of a breakthrough. Also evident in the excerpts is that interviewees seem to experience this "transformation" as having a "before" and "after" quality, which highlights the idea that transformation involves a process, but is experienced as a singular moment after which life is somehow altered.

A breakthrough for me, would be having a totally new thought, a thought that I hadn't had before in my life . . just a totally new thought like, wow, it never occurred to me that it could be this way. But more powerful than just like, "Oh I didn't know elephants took two years to have a baby," I mean, that's very nice, but so what? But a breakthrough in that it could have a real profound effect on how I'm living. For example, well you know, I've lived as that people are stingy and selfish and self-centered and greedy and all this, and maybe they're not that way, then what could--how could that be in my life, like how might I live differently and how might --like how could that be and what would that be like? That was a totally new thought. Before that I was so sure that people are this way. I would never think about why they're that way. So, its a totally new 
thought, but its also really unexpected, its not something that I sat around and worked on.(Jenna 616-34)

[ [The power of a breakthrough is in] revealing something about yourself that you had no idea that you lived in that domain or that paradigm ... Its like, if you didn't know you were in that paradigm then you wouldn't ever sit and take a look at that or study that or think about it. Or question it--because you didn't even know you were like that. . . [I]ts not just in concept, that is actually how the world is. That is your reality ... A paradigm is a reality. . . . so when you have the opportunity for a new reality, that's really powerful because it opens up all these new choices and possibilities that weren't there. They couldn't exist in the paradigm you lived in before. (Jenna, 653-682)

$\square$... in all the work in Landmark, its like, you're always being asked to take on something that's, that's maybe either confronting or challenging or a little bit passed, you know, you barriers or whatever . . . . there's often an element of just getting outside of what you already know or how you have always been, breaking that up a little bit. (Ivan 1385-92)

$\square$ I had definitely a breakthrough in one of my relationships in my life and it was something, it occurring, that could never possibly conceived to have occurred before we went through the Forum, so I think that, if you generalize that it would be the possibility for impossible things to happen. (Mora, 202-213)

$\square$ To go way beyond yourself, you know. To be that thing that you think is impossible, to do the right thing, you know. Or the thing you think is right. You just, the ability to go way beyond your limits, that you've put on yourself. (Mora 227-246)

... when I stood up in front of the room and looked at all the people I was very aware that my fear of that person was connected, absolutely, to a very small physical characteristic that reminded me of somebody that I knew when I was a little tiny kid. . . . And I would just be aware that this person looked like that person, and that person looked like that person and it was all stemming from that. It was so clear. . . . And I think that the Forum gives you the opportunity to figure out how you constructed that thing, you know, what ever it was, you know, it gives everybody the opportunity to examine that and figure it out and get rid of it, you know. Cause everybody's constructed it in a different way. . . (Mora 1083-1128)

$\square$ Its to be able to realize what you're getting--what you are keeping around you 
that you don't have to. You don't want to but you're keeping it around you as a shield, to be a victim, to be a martyr, to be to create disorder in your own life, just so you can deal with the disorder or whatever. (Carl, 888-893)

\section{Transformation From a Fixed Position or Way of Being}

The previous section presented metaphors framing transformation as breaking through a constraining structure. The structure, then, was the from point of transformation and the breaking through aspect described the process or experience of such a transformation. LEC also appears to metaphorically structure the from point of transformation as "a fixed position" and the process or experience of transformation as "giving up" a position or a "shift" from a position (which entails a particular way of being, just as a structure does).

\section{WHAT WE KNOW IS A FIXED POSITION;}

\section{WHAT WE KNOW IS AN IMPEDIMENT TO MOTION}

$\square$ When we start from a stance that something is good and "should be," we find ourselves dealing with life from a particular set of options. . . when we take the opposite stance that something is wrong or that it "shouldn't be," we find ourselves with a different but equally limited set of options. (Sharing, par. 48)

$\square$ If we persist in knowing, we can't go forward. (AC, 608)

$\square$ Maybe have to give up that there is anything out there [reality to be known] except what I speak. (SELP, 271-72)

口 However ambitious or creative our goals, they are essentially limited to what we can predict or project from what we already know or are familiar with. (Sharing, par. 65)

\section{TRANSFORMATION IS GIVING UP A FIXED POSITION}

In the Landmark Forum, we have the opportunity to explore what is available when we give up relating from what we have come to know. . (paragraph 58)

$\square$ Give up that you know. Live with what you don't know. Its just one possibility. If you know how ... you are no longer standing in possibility. (SELP, 96-98)

$\square$ But perhaps it is in stepping outside of what we already know, in giving up what is safe, familiar and comfortable in our relationships, that we can discover entirely new dimensions. ("Sharing," paragraph 53) 


\section{TRANSFORMATION IS A SHIFT (IN BEING)}

$\square$... to transform our relationship to breakdowns from one of being stopped to one of being empowered. (Brkdwn, p. 47, 28-30)

$\square$ Transformation is a shift from being an identity to being an opening. (Brkdwn, p. 64, 24)

$\square$ The goal is to begin shifting who you've been being in your communities. (SELP, 154-55)

$\square$ What if being misunderstood was an opportunity to be fully self-expressed? (SELP, 207-08)

A "fixed position" is an ontological metaphor, although the notion of boundaries is more abstract. A position one holds or maintains does have a sort of boundedness, whether that position is a physical location or a psychological parameter. As long as one persists in maintaining a particular location or holding onto a particular idea, then he or she is unable to move. What is available is limited to what can be seen or done from that position. A fixed position is also an orientation--fixed vs. flexible. If "being that you know" is holding onto or maintaining a position, then transformation is giving up that position, which allows for exploration that was unavailable from the prior position.

Note the coherence between the "structure" and "position" metaphors of knowing; leaving the confining structure of knowing also entails giving up a position. The coherence of these concepts can also be viewed in a key LEC distinction between "being on it" and "getting off it." "Being on it" is a phrase labeling a "fixed" or "stuck" way of being that fits with the metaphor of a "fixed" or rigidly held position. "Being on it" is also framed as being "stuck in" a constraining structure of being called "already always": "When we are 'on it' we are in 'already 
always'--no self-expression there; the world occurs as if there is no possibility" (FIA, 3171-78). The practice of "getting off it" is an act that transforms situations in daily life. "Getting off it" is framed as "giving up" a position, and thus, freeing one's self from being "stuck" and "constrained": "getting off it trades your position for possibility" (FIA, 3182). Leaving the structure or position of "what we know"--or emptying the full container--is moving into or "being with" the unknown. The domain of "what we don't know" or "not knowing" in LEC is possibility--no limits or pre-existing structures but rather, freedom and space to move, to create and to choose.

The metaphor TRANSFORMATION IS A SHIFT IN BEING builds on the idea presented earlier that it is not "knowing" itself that limits, but who one is being within that structure of knowing. One of the most common terms used in association with transformation is "shift": "to change or cause to change place, direction, or position; to exchange: switch; a change from one place, direction or position to another" (Webster's II, 1984, p. 637). Thus, "shift in being" is coherent with "giving up" a particular position or way of being and being open to and discovering other possible ways of being. Both of these also connote movement, as does the earlier metaphors of "breaking through." Movement seems to be a key orientation in the process of transformation.

Transformation is "giving up" a position or way of being: Interview Data. The following excerpts of interview data strongly illustrate interviewees' understanding 
and experience of transformation as a "shift in being." Their comments also feature the earlier presented mapping BEING IS A CONVERSATION. Shifting a way of being involves a particular kind of conversation called "transformation." The last excerpt is a more subtle illustration, suggesting that Leigh's communicative behavior and thus, ways of being with others, has shifted.

$\square$ so I guess you could say that transformation is also about letting go of the past, you know, there's been a particular way of being, that you've had, past tense, and in the moment you give that up, there's an opening for a new way of being to be invented or experienced. . . its not like Landmark corners the market, but, but they are about the conversation that most effectively and powerfully brings about that shift in being. (Ivan 2250-65)

$\square$... there is a shift in your thinking about other people and it is a transformation, I mean that's a word for it, because it really does alter your thinking about your relationships with other people. The way other people occur to you is different after the Forum ... that is certainly a transformation. . . . there can be incredible shifts in relationships just by a mere conversation. . . . they call it the paradigm-shift. ... that's exactly what happens. [For example] my relationship with my ex-wife shifted, ah, and became something completely different, and will be something completely different, from that moment on, through a conversation. (Simon, 1199-1226)

$\square$ I would start with saying that human beings be particular ways, in their lives, you know, we be things in life. Whether its we be frustrated or we be sad or we be excited or we be apathetic--in any given moment there's a way of being that you can say that we have. . . And, transformation is a shift in being. . . . What's common to all [transformative] occurrences is language. I suppose you could say that there are certain conversations, that when held, intentionally, give access to transformation. . . And the Forum is a conversation that when you engage in that conversation, the experience and the process shifts your way of being ... in the specific domain of what you're having a conversation about. (Ivan, 2140-93)

But now I've learned ... how, how to soften it a little bit, and to, ah, ask different questions. Because knowing now, that you're dealing with people's own anxiousness or fears or, that kind of thing, that its going to cause, you 
know, the cause, and then you're gonna get the result of that if, you know, their going to distance themselves from you. So, its helped me, to reevaluate and to kind of, work with people a little bit differently. (Leigh 113-21)

\section{Transformation To a New Life Journey}

Breaking through a constraining structure, giving up a fixed position and other forms of transcending the limits of knowing, all allow the opportunity to move beyond that which is familiar in life and explore. Movement and exploration begin to suggest an instance of the conventional LIFE IS A JOURNEY metaphor (e.g. "I don't know what path my life will take now," "I've been feeling lost," "This project is going to slow me down") (Lakoff \& Johnson, 1980). The "breaking free" and "giving up a position" metaphoric clusters structure the paradigm-shift--the initiation of the journey--while the journey metaphor indicates the way transformation can occur in one's life ongoingly.

\section{TRANSFORMATION IS A JOURNEY OF EXPLORATION AND DISCOVERY}

$\square$ But perhaps it is in stepping outside of what we already know, in giving up what is safe, familiar and comfortable in our relationships, that we can discover entirely new dimensions of others, of ourselves, and of what is possible. ("Sharing," paragraph 53)

$\square$ You are able to leave the past in the past, to step beyond the limits of your identity, and to move into a future mapped by what is possible, not just by what has been. (Forum brochure, 2705-2709)

$\square$ This shift, or transformation, is not a one-time event, but a moment-to-moment approach to being alive, an ongoing access to a previously untapped dimension. . (SEF brochure)

$\square$ Undauntable people at play ... on an unknowing, uncharted exploration of limitless growth and development. (LEC International Assisting Charter)

The structural metaphor TRANSFORMATION IS A JOURNEY OF

EXPLORATION AND DISCOVERY is a novel adaption of the conventional LIFE 
IS A JOURNEY mapping. Conventional occurrences of the LIFE IS A JOURNEY metaphor are prevalent in LEC, which is not unusual considering the nature of the work that they do; transformational work suggests significant alterations in ways of living, and thus, alterations of the life journey. What is of more interest in this study is LEC's adaption of this conventional metaphor to meet their own purposes. The "journey" mapping helps LEC structure aspects of life that entail the progression of time, goals, problems, and achievements. LEC modifies the conventional mapping to incorporate the impact transformation has on participants' lives. Being a human being entails having experiences, making conclusions, learning and acquiring knowledge, thus, living a life of transformation entails a continuous process of stepping out of "what we think we know" and into possibility, and "from there," creating, inventing, generating life. In other words, transformation is an ongoing "conversation for possibility," in which individuals choose and create ways of being not determined by the past.

The notion of destination in LEC's adaption of the journey metaphor is different than in the conventional usage, although it is difficult to articulate. The difference stems from the idea that prior to the Forum, the destination of the journey was being determined by the past--what had come before was limiting what could be. The destination was just some version of what had already been. Once "we put the past in the past," the future is "empty" and the destination is "open to being invented in 
any number of ways." Invention, of course, is a process and product of conversation.

On such a journey, "what we think we know" would always be subject to transformation, requiring one develop an ability or capacity to distinguish at any given moment "where one is" on the journey as well as an eternal openness to consider other possibilities. This metaphor highlights the sense of limitless possibility, but distracts our attention from the potential for impermanence and instability introduced by a life that is always subject to change. It also distracts our attention from the fact that LEC's "technology" also becomes something known, and thus, contains the potential to limit ways of being us as much as any other form of knowing.

The metaphors we have explored so far do suggest that LEC's intended outcome of participation in the Forum (and the Curriculum for Living) is a form of paradigm-shift called "transformation." The metaphoric structuring of "transformation" suggests an open-ended and relatively unspecified experience. For example, transformation is commonly described as discovering or opening "new dimensions." This might suggest an expansion or extension of one's paradigm or worldview--being able to see more and differently than before. A dimension can be defined as "a measure of extension, especially height, width, or length" (Webster's II. 1984, p. 198). Opening "new dimensions of living and being" then, suggests expanding the previous boundaries of those areas, creating more space, more room for "movement" without specifying an amount or degree of expansion, space or 
movement. "Opening a new dimension" for one person may involve making a risky career move while for another "opening a new dimension" might occur as forgiving someone for a perceived wrong against them.

Abstract language such as shifting, beyond, open, giving up, stepping out/into, standing outside, new dimensions, explore, discover, breaking through all suggest a sense of movement in time and space, flexibility, overcoming obstacles, as well as a sense of excitement or adventure. Thus, the quality of transformation is suggested, but the specifics are left open to individual interpretation and experience. Sackmann (1989) notes that "adaptive metaphors" are particularly suited to mapping "a transformative process whose outcome cannot be specified at the outset. [Adaptive metaphors] connote a continuing process of search, taking action, and adjustments toward 'fuzzy' goals which may change during the process" (p. 471). This seems particularly relevant in the case of LEC where "transformation" must be available to all participants and adaptable to their individual life paths. The fact that transformation is not a one-time event, but an "ongoing access to a previously untapped dimension" (Sharing the Forum, paragraph 43) also points to the need for the concept or transformation to remain open to individual interpretation. If as suggested "The Landmark Curriculum for Living leaves you with the competency to ongoingly generate your transformation in life" (Curriculum for Living doc.), then transformation must be applicable to my life and the circumstances of my life. 
One thing LEC does specify as being a part of journey resulting from transformation is "possibility." Breaking through, giving up, shifting--all these experiences of transformation include the bringing of "possibility" to one's life. Like the other open-ended and abstract language associated with the outcomes of transformation and living a life of transformation, "possibility" may be expressed and experienced very differently by different people. Generally, possibility includes the qualities of being "an opening," "new," "beyond" the known or previously expected, unfulfilled or "empty," and "flexible." These orientations will be discussed more in the final section on LEC's orientational metaphors.

Transformation is a Journey: Interview Data The metaphoric structuring of transformation frames it as breaking out of or through a constraining structure and/or giving up or shifting one's self from a fixed or stuck position. These metaphors structure the from point, process and experience of transformation. The to aspect of transformation is framed as an open-ended journey that involves movement through the unknown, discovering or creating new dimensions, and generally speaking, the experience of "possibility." These themes are evident in the interview excerpts included here. Conventional journey metaphor language is plentiful in these excerpts, but notice the somewhat subtle hints of LEC derived meaning structures for transformation that conventional language is combined with. Notice also several references to LEC's role in initiating or guiding some interviewees' journey. 
in the SELP I wanted to take on something that would put me in a place I've never been before. (Ivan 1393-94)

its focused by the leader who knows the possibility that can ... happen. And it just gets created by the people there. . . you know, how do you go from something you don't know about, into that being your reality?. . . You have to somehow be shown the way or have a breakthrough, But in our Forum we were shown the way by the leader, that's obviously what happened. (Mora, 265-276)

- ... you see, we're all adults, and we think that as, as we, as we're growing up, as we're going to school, and college, and then get married, and have kids, that we're slowly gaining all this knowledge and pretty soon you get to the point where "I know everything. I pretty much can run my life now." And you know, "what ever occurs I'll be able to handle it." And, and so, you become very aloof and you become cocky and stubborn and when you can drop all that . . that facade.... see the wonderful thing about being coachable at the Forum, at Landmark, is that, you become more coachable, the further you proceed because Landmark didn't steer you wrong in the Forum, and they didn't steer you wrong in the seminar, and they didn't steer you wrong in the Advanced course--they promise you certain things . . they give you a list of promises, and they fulfill their promises. . . they're not going to steer me wrong. (Simon 906-32)

- Once--you've gotta get past that, before you can move on, and ah, once you really become at peace with yourself that's when you can really start to move forward and that's what I got out of the Forum. That's one of the great things that I got--I became okay with who I am. And I think that, not only that, but you begin, you begin, to become okay with everybody around you. (Simon 580-96)

And transformation is when there is genuine growth, you're not just running in a circle, you're actually trying something new here and then something new and something new. You're actually trying new things--new ways of living, new ways of communicating, new, new whatever. But you're actually doing new things all the time versus just repeating. Like my relationship with A . . Our relationship where its going is not where I've ever gone before. . . percentage wize, you know, there's more new than old there. . . And transformation is when there is real growth in any area, whatever that is. (Jenna 962-83)

$\square$ But when you're paying attention to this person, and really, trying to appreciate them and getting--being completely present to the idea that they're expressing it 
just, elevates the possibility of what can come out of that into something... beyond what you know now, you know. (Mora, 665-79)

$\square$ I think its transforming yourself from the quagmire you create around yourself to being able to walk on dry land. A lot of people they just sit there and stew. And that's the thing that, you know, the if you have a gripe with somebody or something or your parent or a um, ah, you know, a former partner or 3omething, that you're--you're the one dragging that along, they aren't spending everyday of their life trying to make sure that you're dragging it with you. Typically, but ah, and I think its that sort of transformation--to be able to step out of that--you're own little poor pitiful me shell and, uh, carry on. And move. (Carl 874-884)

Evident in these excerpts is the notion that transformation has involved "shifts in being" for the interviewees. Also highly evident in these excerpts are examples of movement and movement "beyond" perceived limitations of some kind and being in or putting one's self in a previously unknown or new locations. One can get a sense of the consequentiality of participating in LEC's courses in the lives of these interviewees.

The metaphor clusters in the areas of conversation, being and transformation, while only a portion of LEC's entire metaphorical system, suggest the complexity and systematicity of the overall system of which they are a part. The next section presents some of the key orientational metaphors that organize LEC's metaphoric system and demonstrate the systematicity of that system.

\section{Orientational Metaphor}

Rather than structuring one concept in terms of another, orientational metaphors organize relationships among whole systems of a culture's most 
fundamental concepts. For this reason, I chose to place the section on orientational metaphor last, since one needs a sense of at least a significant portion of the system before being able to see how orientational metaphors organize that system. Lakoff and Johnson (1980) note that there is typically external systematicity among the various spatialization metaphors of a culture, which "defines coherence among them" (p. 18). Deetz (1984) notes that "the systematicity of metaphors is easily observed in the orientation metaphors" which "rest in what might be called archetypal orientations that integrate a whole group of different experiences" ( $p$. 219). Orientational metaphors also organize and prioritize the value system of a culture with the metaphorical system:

The most fundamental values in a culture will be coherent with the metaphorical structure of the most fundamental concepts in the culture. . . which values are given priority is partly a matter of the subculture one lives in. . The various subcultures of a mainstream culture share basic values, but give them different priorities. (Lakoff \& Johnson, 1980, p. 17; 23)

For example, generally speaking, in our own culture GOOD IS UP which gives an UP orientation to concepts associated with general well-being (HAPPY IS UP, ALIVE IS UP, CONTROL IS UP, STATUS IS UP, etc.) Now consider an example of U.S. culture values that are coherent with the GOOD IS UP orientation. "More is better" is coherent with MORE IS UP and GOOD IS UP, while "less is better" is not (Lakoff \& Johnson, 1980). Unless of course we are talking about "cost" or "expense." In this case "less is better," which is still coherent with MORE IS UP and GOOD IS UP if you consider that if the cost is less, you still have more currency or 
resources left afterwards. The "less is better" example demonstrates a difference based on the priority given to particular values by a culture.

The spatio-temporal orientations that comprise LEC's orientational metaphors do function in the U.S. mainstream culture within which LEC's cultural system exists. U.S. culture orientations are also found in LEC's system of spatio-temporal orientations. However, which orientations are most salient and most valued tends to differ. For example, GOOD IS UP, which is a highly prioritized orientation in mainstream culture, can be found in LEC's discourse ("What are you up to?"--an example of DOING/ACTIVITY IS UP which is coherent with LIFE/HEALTH IS UP) but the UP orientation is not given as high a prioritization in LEC. Therefore, the UP orientation did not emerge prominently in the LEC derived discourse of this study. The orientation OPEN, on the other hand, seems to be one of LEC's most highly prioritized orientations (to be discussed in more detail momentarily). The OPEN orientation can be found to function in the U.S. mainstream conventionalized metaphoric system (e.g. "She is really open to new ideas"--evidence of OPEN IS GOOD), it does not figure as prominently as a key organizing orientation for the system as a whole.

LEC's orientational metaphors thus, re-frame mainstream culture concepts in order to construct the prioritization and coherence of their own metaphoric and value systems. The table below is a depiction of how LEC organizes the relationships among what they call "the design principles of human being." 


\begin{tabular}{|c|c|c|c|c|}
\hline Given & Orientation & Transioriagtion & Orientation & Invented \\
\hline Already Existing & Closed & shiti & Open & Not Yet Existing \\
\hline Knowing & Stuck & Beyoud & Free & Not Know \\
\hline Right/Wrong & Stopped & Conversation & Movement & Interpretation \\
\hline Position & Fixed & Brealithrough & Flexible & Location \\
\hline Automatic & Full & Ging 1 ) & Empty & Choice \\
\hline Reactive & Not Present/ & out ortuto & Present/ & Proactive \\
\hline Rackets & Past & $10 \cos \theta$ & Future & Creating \\
\hline Win Formula & Old & OHothag is hing & New & Possibility \\
\hline Withholding & & $\%$ & & Sharing \\
\hline On It & & & & Off It \\
\hline
\end{tabular}

The two outer columns list samples of fundamental concepts LEC frames as characterizing two domains of being. The far left column represents those "design principles" and resultant ways of being that are "already always there" and can be considered "given." Left undistinguished, these aspects of human design are said to limit the potential for human actualization in the world. These "given" ways of being are an "inherited" part of the design of human "being." That is, there is "nothing wrong" with us that needs to be "fixed"--human beings are not broken or defective. The goal is to gain awareness of these potentially limiting ways of being, thereby providing the opportunity to utilize fully another "inherited" aspect of the design of human "being": the ability to create through language and conversation, ways of being not "given by the past." The concepts in the far right column are those ways of being LEC reveals as part of the "new dimensions" of living and being in which human beings generate or invent their existence, rather than being "used" (i.e. controlled) by what they inherited. These concepts are also part of the design of 
human being and access to them is distinguishing individual power to create through conversation (i.e. language use, speaking, and listening).

The next two columns inward (labeled "orientation") illustrate the spatiotemporal orientations LEC associates with the domains of being represented in the outer columns. The dimensions created by juxtaposing the two orientation columns (e.g. open/closed is a dimension) provide the structure for organizing and prioritizing the metaphorical concepts and values in LEC cultural system. In the center column are examples of concepts associated with "transformation": getting from the "already-always" ways of being (structures, positions) we "inherit" to inventing new ways of being that offer possibility and choice. One shifts from being in a "racket" to creating a possibility or one has a breakthrough or gives up a position, etc.

Openness is LEC's key orientational metaphor; without openness, transformation is not possible. In LEC, OPEN IS GOOD and most of LEC's other spatio-temporal orientations are found to be coherent with OPEN. Openness entails the psychological orientation of being "open" to other possibilities, being open to other ideas, open to shifting, open to others (e.g. sharing), and open to the unknown. The orientation OPEN is exhibits external systematicity with all of the concepts in both outer columns of the table above. For example:

NOT YET EXISTING IS OPEN; ALREADY-EXISTING IS CLOSED NOT KNOWING IS OPEN; KNOWING IS CLOSED INTERPRETATION IS OPEN; ASSESSMENT (right/wrong) IS CLOSED 


\section{LOCATION IS OPEN; POSITION IS CLOSED}

CHOICE IS OPEN; AUTOMATIC IS CLOSED

SHARING IS OPEN; WITHHOLDING IS CLOSED

This coherence with OPEN holds true throughout the concepts in the table. In addition, the OPEN orientation is in some way related to or coherent with all of the other orientations. For example, EMPTY/FULL are related to OPEN/CLOSED and both are related to freedom of movement--when something is empty there is room or openness for other possibilities and for movement, creation and action. When something is full there is not. OPEN is also coherent with THE PRESENT and FUTURE, while THE PAST is CLOSED. OPEN allows for newness, so OPEN is coherent with NEW.

Movement is another highly valued orientation in LEC. Being "stuck" is associated with automatic ways of being (rackets and winning formulas), holding onto a position, withholding, equating interpretation with truth, etc. Many of the other orientational metaphors suggest the feeling of movement or provide the possibility for movement. If something or someone is "free," movement and choice are possible. A location is different form being "stuck" in a position, as a location connotes more flexible boundaries, allowing room for movement. Movement also seems to be valued in mainstream U.S. culture, but primarily as it relates to upward or forward progress. While upward or forward progress seem to valued in LEC as well (e.g. "that's when you can really start to move forward and that's what I got out of the Forum," Simon, 582-86), LEC's value of movement is more strongly 
associated with and valued as the notion of "flexibility" (e.g. "You are free if you can go back and forth between points of view," AC, 370-71). This kind of "flexible movement" is not as highly prioritized in mainstream culture--especially in the realm of ideas and actions--where "right" and "wrong" are typically held to exist as "real." In LEC "right" and "wrong" exist, but are recognized as human inventions, as interpretations, which allows more flexibility of movement--especially psychological and intellectual.

The mappings and metaphoric linguistic expressions below demonstrate many of LEC's orientational metaphors in action and interaction. The collections of metaphoric linguistic expressions in this section include some brief excerpts from interview data. Reviewing metaphoric linguistic expressions in previous sections will review many more examples of LEC's system of orientational metaphors.

\section{POSSIBILITY IS AN OPENING; POSSIBILITY IS EMPTY} FULL IS NO POSSIBILITY; EMPTY IS POSSIBILITY

$\square$ What opened up out of [doing the homework] was. . (AC, 275)

$\square$... create openings through communication, sharing. (FIA, 3454-55)

$\square$ Enrollment: A conversation that leaves another in an opening; generating possibility in another's listening such that they step into that possibility committedly and act. (AC, 192-195)

$\square$ You're open to the possibility that impossible things can happen. (Mora 9971001)

$\square$ In the Forum, the last day that, you know, that life is empty and meaningless and what you get out of the Forum is you pay two hundred and ninety dollars for is nothing, you get nothing, which is possibility. (Ivan 1138-41)

$\square$ in this model you are an ontological space for listening, a clearing for the conversation to take place in. (CAP, 9/96)

$\square$ none of us come to any situation completely empty of thoughts, ideas or points of view. There is a way we look and listen . . that is already in place.

("Sharing," par. 71) 


\section{TRANSFORMATION IS AN OPENING; A SHIFT CREATES AN OPENING}

$\square$ as people share what they are working on, what's opening up for them since completing the Forum. (Brkdwn, p. 45, 5-9)

$\square$ Transformation is $a$ shift from being an identity to being an opening. (Brkdwn, p. 64, line 24)

$\square$ In accessing the roots of your own power and spirit, you find not an answer but an opening (Forum brochure, 2725-28)

$\square$ We usually listen for, rather than to. Giving up our concerns (that something is wrong, something to fix, etc.) creates space for listening (CAP, 9/96)

$\square$ So that's what the Forum did for me, it just opened my eyes to a whole nother arena of interacting with people. (Leigh, 190-92)

\section{THE FUTURE IS OPEN; THE PAST IS CLOSED}

THE FUTURE IS EMPTY; THE PAST IS FULL

$\square$ To put the past in the past and open a future not given by the past. (FIA 343031)

$\square$ So, so I guess you could say that transformation is also about letting go of the past ... and in the moment you give that up, there's an opening for a new way of being to be invented or experienced. (Ivan, 2250-55)

$\square$ The Advanced Course is about generating and speaking a future into existence from nothing. (Ivan 985-87)

$\square$ The more you know who you are, the less possibility--based in the past (AC, 38-40)

$\square$ The listening we are is filled with what we know--no room in the cup. (AC, 4950)

WITHHOLDING IS NOT PRESENT; WITHHOLDING IS CLOSED, STUCK, STOPPED; SHARING IS MOVEMENT; SHARING IS OPEN

C Source of all the suffering in your life: Withholding . . more withholding, ... more stuck in private conversation--not present. (AC, 574-77)

$\square$ What ever stops you from sharing/being self is exactly what stops you in life. (AC, 449-50)

$\square$ Everything we are withholding keeps stuck in the interim test! (AC, 328)

$\square$ This work lives in sharing, in conversation; when that stops, so does transformation. (Brkdwn, p. 65, 20-21)

$\square$... its only when they just, surrender, and become coachable, and start sharing (Simon, 654-56) 
BEING "ON IT" IS THE PAST, FULL,

\section{A POSITION IS CLOSED; A POSITION IS STUCK}

GIVING UP A POSITION IS NEW;

$\square$ What ever can't try on is stuckness" (AC, 129)

$\square$ They seem positional--closed--They are on it, stuck. (FIA, 3170-71)

$\square$ When we are "on it" we are in "already always"--no self-expression there;

When we are "on it," "empty and meaningless" and "life as a conversation" are missing/absent (FIA, 3177-80)

$\square$ Willing to give up being right for being related in a new way? (FIA 3375)

$\square$ What shifts an issue from stuck to free is completing [communication] it . . dissolves positions/shoulds. (FIA, 3356-59)

\section{REALITY IS FLEXIBLE; FLEXIBLE IS OPEN}

$\square$ we can reference ourselves against something that does not yet exist . . and from there open up a whole new dimension of living and being." Standing there, standing in what is possible, the world that becomes available" ("Sharing," 60)

$\square$ In The Landmark Forum we see what we know, what we think, and even "who we are" may not be fixed and set in place, but may be completely flexible and open to being invented in a number of ways. . . (Forum brochure)

One can begin to see clearly the external and internal systematicity among the concepts and the orientational system. That is, most of them are consistent with one another. I can just as easily say "not knowing is freedom" or "not knowing is the present" or "creating is in the present" or "choice is movement," etc.

As mentioned earlier in this section, the orientations of "up" and "movement" are prioritized differently in U.S. mainstream culture than in LEC. Discourse derived from LEC courses and artifacts did not reveal high prioritization of "up" and "forward" movement orientations. Such references were more frequently in the interview data, along with discourse supporting LEC orientations. For example:

$\square$ "I've always had a very positive attitude on life, and that has been stepped up to a different level." (Leigh, 756-57)

$\square$ "Its just stepped me up, to . . really enrich things." (Leigh, 1044-48) 
(] "The wonderful thing about being coachable at the Forum ... is that, you become more coachable, the further you proceed" (Simon, 917-19).

Examples such as this might suggest the intersection of the two different cultures' orientational and value systems.

The values emerging from analysis as differently prioritized by LEC revolved primarily around openness (including emptiness and space)--"open is better," "empty is better," etc.). Openness and emptiness create space or freedom for movement and flexibility of movement, which is another highly valued orientation in LEC-"freedom is movement/flexibility," "movement is good." Openness and emptiness are more highly prioritized however, since without them, the movement and flexibility valued in LEC is not possible.

Another set of orientations that seem to be more differently prioritized in LEC are "the past," "the present" and "the future." For example, in LEC, "the past" is acknowledged as having some value as a reference point, but overall, is seen as something that limits possibility in human living, at least when humans allow "the past," wittingly or unwittingly, to dictate their actions in the present (a product of "the past" being undistinguished as "the past"). In mainstream culture "the past" is typically valued as something to learn from ("I'll never let that happen again!") or build on ("Those traditions are the foundation of our company") or as knowledge about how the world is ("I already know what she'll say--that's just how she is"). The degree to which we define our present and future based on the past, and the 
degree to which this limits possibility, seems to be less important in mainstream culture, while in LEC, this is a key element in the overall system.

"The present" is more highly prioritized and valued in LEC than mainstream U.S. culture. "Getting present" and "being present" are key orientations in the process of transformation and the creation of possibility, which are also highly valued concepts in this culture. U.S. culture, while acknowledging "the present," seems to more highly value "the future" as framed within a goal of progress. That is, mainstream culture seems to take "the present" for granted, as a given but rather unimportant temporal experience on the way to "the future." "The future" is also valued in LEC, but that value is dependent upon having a future that is "open to being invented" which requires taking "the past" out of "the future" (see pp. 12628). Otherwise, humans are just living into some version of their pasts projected into the future, leaving the future already full, already determined. In mainstream culture, it is important to be able to predict and control "the future," which relies then on "what we already know" and "the past," leaving little room for possibility. As mentioned earlier, mainstream culture also functions as if "right" and "wrong" are "real," and therefore, there is a "right" way and a "wrong" way for the future to unfold. In LEC, the labels of "right" and "wrong" are seen to limit choices people make about their futures, and thus, limits freedom of movement and the possibility for the "new." 
We can also see the influence of the LEC's orientational prioritizations on the larger metaphoric system. The root-metaphor LIFE IS EMPTY AND MEANINGLESS is a good example. Notice the highly valued orientation of "empty." Life is an empty container, which can be filled in any number of possible ways. Thus, "empty is good" because there is openness, room or space to create. Human beings may be "meaning-making machines" (another root-metaphor), but because "life is empty and meaningless," human beings can choose what meaning to make; "choice is open." Metaphors in the area of language and conversation reveal that LEC highly values communication as a creative act. Human awareness of communication as something that transcends temporal boundaries is also highly valued. Communication is valued as a powerful access to all other things that are valued in this community. And, while LEC may not label automatic or ineffective ways of being as "wrong," they are clearly not valued as evidenced by the orientations associated with them: stuck, fixed, closed, full, stopped, not present.

The examples I have provided represent only a fraction of the ways in which orientational metaphor organize the entirety of LEC's metaphoric system. Reviewing previous sections of this chapter in light of this section would likely reveal a host of examples of the influence of orientational metaphor in organizing the overall system.

\section{Chapter Summary}

This chapter presented LEC's triadic root-metaphor structure: LIFE IS EMPTY AND MEANINGLESS, HUMAN BEINGS ARE MEANING-MAKING 
MACHINES and LIFE IS A CONVERSATION. After briefly looking at these rootmetaphors, LEC's emergent system of metaphors was presented in four sections: conversation, being, transformation and orientational metaphors. The experiential structure provided by these metaphors was explored and excerpts from interview transcripts were integrated to provide a glimpse of the metaphoric structure as it occurs in the discourse of cultural participants. Chapter six will discuss the implications of this study's findings, present the limitations of this study and implications for future research. 


\section{CHAPTER SIX--DISCUSSION \& CONCLUSION}

The purpose of this study was to explore the intersection of metaphor, transformation and culture by revealing the meaning structures within the cultural context of Landmark Education Corporation. This chapter will review the research questions posed and discuss the findings generated toward addressing those questions. Also presented in this chapter is a discussion addressing that which the abstracted metaphoric system tends to obscure, some inherent inconsistencies in the overall system, and general concerns that emerged with the findings. Finally, the limitations of this study and implications for future research will be discussed.

\section{Discussion of Findings}

\section{Research Questions \& Review of Findings}

The following research guided this study:

1. What constitutes the metaphorical system embedded in and abstractable from various LEC texts?

2. What is the experiential structure provided by LEC's metaphoric system?

3. How do metaphors and their experiential structure manifest in the discourse and lived experiences of LEC's cultural members? How do LEC participants make metaphors meaningful in their everyday lives?

4. What are the meaning structures associated with people's experiences of transformation, empowerment, and community?

This study has successfully revealed at least a portion of the metaphoric system associated with LEC's "culture of participation." With analysis emerged the realization that LEC has developed a complex system of meanings situated in a comprehensive and, in many ways, alternative worldview. Comprehension of the 
emerging metaphors seemed to actually require an understanding of the larger experiential structure (i.e. worldview) being produced and reproduced in this culture's discourse. This can be attributed to the idea that metaphors, as part of a culture's discourse, actively "produce their own conditions for being understood" (Deetz, 1984, p. 219). Complicating the need for familiarity with LEC's worldview was an issue of translation, as the emerging metaphors are situated in a "way of speaking" with a unique vocabulary and highly developed system of shared meanings and experiences. This is congruent with Berger and Luckmann's (1966) contention that understanding any shared reality is fundamentally tied to understanding the language of that reality. Explicating large portions of LEC's worldview was both a finding of this study as well as an analytic process, providing a necessary "thick description" of the experiential structure emanating from and supporting the metaphoric system. This lends support to the idea that language, and thus metaphors, are more than objects for expressing experience, they are manifestations of how a culture thinks (Deetz, 1984).

These findings indicate that research questions one and two are in fact, inseparable. The need for an understanding of the larger experiential structure within which a culture's metaphors operate becomes especially crucial if the metaphor analysis is being performed in an unfamiliar culture or if the results of the analysis will need to be comprehensible to a population unfamiliar with the culture. 
Analysis revealed that LEC's worldview emanates from a triad of rootmetaphors: LIFE IS EMPTY AND MEANINGLESS, HUMAN BEINGS ARE MEANING-MAKING MACHINES, and LIFE/LIVING IS A CONVERSATION. Also emerging from analysis was a portion of LEC's entire metaphoric system organized into four clusters of the culture's most salient metaphors: (1) metaphors structuring concepts of language and conversation; (2) metaphors structuring concepts of human "being"; (3) metaphors structuring concepts of transformation; and (4) orientational metaphors organizing spatio-temporal relationships among the metaphoric concepts and making the overall system coherent with the culture's value system. Adopting and acting on new metaphors such as these can "give new meaning to our pasts, to our daily activities, and to what we know and believe" (Lakoff \& Johnson, 1980, p. 139), which becomes especially important when the new metaphors are part of structuring a paradigm-shift, a "resocialization" regarding reality. LEC's metaphoric system and worldview provide the "legitimating apparatus" for a "resocialization" to an alternate social reality which is experienced as transformation and results in reinterpreting the meanings and social relationships of conventional, mainstream "reality" within the "new reality" (Berger \& Luckmann, 1966).

The system created through the combination of these metaphors privileges language and communication (conversation) as a process of creation and frames all modes of human "being" as products of this creation. From this perspective, the 
realities of the world we live in exist only as a product of our conversations. In adopting this constructivist perspective, LEC and its participants elevate the significance of communication and locate power within individuals who actively participate in creating and maintaining the realities they live in. Participants in LEC's experiential structure begin to develop an awareness of the power of the words they choose and the ways in which they engage in communication with others:

$\square \quad$ I've never thought that it was very important how you said things, before. You know, like the difference between have or want, or should or will, or--it just the words you choose, I just didn't think it was that big of a deal. And now I really see that it does create your reality. I mean however you say it is, it really is that way (Jenna, 1156-62)

$\square$ But when you're paying attention to this person, and really, trying to appreciate them and getting--being completely present to the idea that they're expressing it just, elevates the possibility of what can come out of that into something ... beyond what you know now, you know. And that can only happen, I--I believe that it can only happen--when you are totally present and totally paying attention in a completely nonjudgmental way and just saying, like, anything can happen from this conversation that I'm having with you right now, you know. And that was just, amazing. The possibility of the words that you choose and the language you use to make the future happen. (Mora 663-682)

In this experiential structure the world is not imbued with the meaning and purpose of human existence. Instead, human beings as "meaning-making machines" who create reality in and through communication become aware of their own authorship in shaping their experience, as well as freedom and responsibility to choose. Instead of searching the external world for direction and meaning, individuals choosing to participate in this system must look inside themselves and ask the question: "What will I have my life be about?" The key to creating one's 
reality is first to speak it--to choose it and begin the conversation that will bring it into existence.

The highly constructivist approach to and value of communication within LEC's cultural system occurs as a distinct contrast to mainstream culture's conceptualization of communication as a process of transferring "real" units of meaning via the conduit of language (Reddy, 1993). From the "conduit" perspective, communication is putting meaning into the appropriate word containers and sending these containers "across" to receivers who then simply extract the meaning from the words. Reddy also notes that the "conduit" framework implies that successful communication should be effortless and what effort is required is primarily the responsibility of the speaker, trivializing the role of listening. This framework actually allows all participants in communicative processes to avoid taking responsibility for successful or effective communicative transactions.

LEC's metaphoric system reframes mainstream conceptualizations of communication, creating an alternative perspective that views communication as the very essence of human "being." This is reflected in the meta-language used within LEC's symbolic system to talk about communication and communicative processes. Consider, for example, language used to talk about "meaning" in LEC. Meaning is "brought into being," "generated," "created," and "invented" by conversational participants; meaning "occurs" for people and "arises" through conversation. 
While much, if not most, of the meta-language in LEC indicates constructivist frames for communication, there are linguistic occurrences that seem reminiscent of the "conduit" metaphor. For example, one common linguistic practice is that communicators in LEC frequently use the phrase "I got it" or "got it" to indicate understanding of something being communicated to them. This linguistic habit suggests the notion of accurately receiving meaning from another. Reddy (1993) would likely explain linguistic occurrences such as this as instances of "frame conflict." According to Reddy, while we may be able to think and speak in terms of an alternative frame for communication, the logic of the "conduit" metaphor is deeply embedded in the English language:

The logic of the [conduit] framework runs like threads in many directions through the syntactic and semantic fabric of our speech habits. Merely becoming cognizant of this in no way alters the situation. Nor does it appear that one can adopt a new framework and develop it while ignoring the cloth of the language. (pp. 176-77)

The influence of the "conduit" metaphor may be difficult to completely avoid, however, LEC's overall metaphoric system and experiential structure represents an active and comprehensive alternative frame for conceptualizing communication. Adopting this alternative framework seems to result in a heightened appreciation for the effort involved in communication and a heightened sense of responsibility in performing as speakers and listeners. As mentioned earlier, adopting LEC's almost radical-constructivist meaning structures also appears to contribute to participants' experience of personal empowerment. 
The literature presented on empowerment in chapter two consistently makes reference to a need for human beings to experience a transformation of some kind in order to achieve, acquire or recover a sense of control, as well as the practical power, to effect their own lives (Rappaport, 1984; Katz, 1984; Lerner, 1986). This suggests that the perceived norm, the status quo, is that people experience themselves as powerless, as victims, at the mercy of world not of their own making. Lerner (1986) contends that the undermining of human actualization and the experience of fragmentation and isolation contributing to "surplus powerlessness" are part of what human beings inherit with the world they are born into, a world that is already constituted and reified in language: "The very act of entering the linguistic system means entering into a world of meanings in which the fundamental fairness of how things are set up is assumed and asserted" (p. 147). Lerner asserts before transformation can take place, before any kind of empowering structures can be created, these reified conditions must be unconcealed and addressed (Lerner, 1986). Only then can a "conversation for possibility" truly take place.

What is it within the experiential structure of LEC that provides for or facilitates the transformation necessary for participants' to experience themselves as empowered? One potential influence is that participation in LEC seems to provide people with an awareness of their own "authorship in the world" (Berger \& Luckmann, 1966). The practice called "distinguishing" in LEC seems to be instrumental in unconcealing reified and taken-for-granted structures (e.g. 
"conversations") that disempower people personally and socially. Distinguishing shifts figure/ground relationships and provides access to creating more empowering structures. LEC's experiential structure is infused with the idea that human beings create and recreate their realities through language and conversation. With this realization individuals can begin to see themselves as consciously participating in the shaping and maintaining of the everyday realities of their world. The themes consistently tied to empowerment in the discourse of LEC participants include personal responsibility, freedom, the power to choose, and the power to create through their speaking and listening. For example, LEC participants develop an awareness of the role their internal and private conversations and language use play in shaping their experience and actions:

$\square$ See that's my old conversation coming up again (Mora, 466)

$\square$... conversations that stop you just don't make a difference. Rackets. . . That rackets simply don't make a difference in the world. Its not good or bad, its just that they don't make a difference in the world. So I could see that I had a racket about exercising and that that conversation was not making any difference in my life. (Ivan 763-774)

The self-talk that goes on, where nobody sees it or hears it, that talk you out of everything. That talk you, into believing that this person is out to get you, but you don't have any facts to prove it. And so its, been very empowering, well, what was really said, or what really happened. (Leigh 995-1000)

$\square \quad$... and something else that was said in the Forum is that "life is a conversation." And when you can distinguish the conversations that you have, the automatic ones that come from, lets say your past, or comes from something that happened, you know, yesterday or the day before or when you were three years old--when you can distinguish that were started then, as conversations, you don't have to automatically be run by them. You don't have 
to take them as, the quote unquote, "real" thing. ... You then have the power, to choose another conversation to have about the matter. (Ivan 436-72)

you know with language, how disempowering we can become, ah, we disempower ourselves. That was one of the big things I picked up in the S.E.L.P, was-Oh yeah, we pull the rug out from under ourselves all the time, ah, and, and make ourselves less powerful then we can be. (Simon 1647-52)

Examples like these occur throughout the interview data. Kieffer (1984) observed that one key associated with people's experience of empowerment was developing an awareness of one's internal dialogue and learning to use that dialogue constructively. If one truly internalizes the idea that "life is a conversation," than all forms of conversation come to the foreground of awareness. Communication becomes more than a medium of exchange or thoughts in one's head. All forms of communication become acknowledged as creation itself.

Another consistent connection made in the empowerment literature is the need for a sense of connection or community with other people--that somehow the experience of isolation and fragmentation contribute to or exacerbate the perception that one is without significant power to create the kind of life, or the kind of world, they have dreamed about (Lerner, 1986; Rappaport, 1984; Katz, 1984). Lerner (1986) believes that as long as people accept fragmentation and isolation as just part of the way the world is, there is no possibility for change or actions that would facilitate change. Not surprisingly, creating community that counters reified conditions and experiences of isolation is the key to facilitating empowerment that leads to personal and social action (Lerner 1986). Katz (1984) notes that the 
possibility of being empowered and empowering others would be more fully realized if developed within an alternative paradigm he calls a "synergistic community" (see pp. 49-50). Such communities he contends, can be established using "education as transformation" to create "rituals of transformation":

in which participants experience a transformation of consciousness. This is not necessarily a major, intense or radical alteration of consciousness. Nor is the ritual necessarily a dramatic or clearly demarcated event. ... This transformation seems to bring on a (new) way of experiencing self as embedded in and expressive of community. . To establish the empowering environment which is found in a synergistic community requires a radical paradigm shift--a major shift in the way persons experience meaning and process and interpret data. (p. 208, 222)

In many ways, LEC's courses seem to fit these descriptions. Certainly participants, if they begin to adopt or find coordination with the new metaphoric frames, experience a shift in what meaning is and develop an awareness of their own and others perceptual and interpretive processes. Consider, for example, the rootmetaphors LIFE IS EMPTY AND MEANINGLESS, HUMAN BEINGS ARE MEANING-MAKING MACHINES, and LIFE IS A CONVERSATION. The inference patterns of these metaphors alone clearly suggest a very different view of and approach to what reality is. LEC's experiential structure includes the notion that "what we think we know" is a creation, a product of interpretation. This perspective would facilitate the ability to "recognize descriptions as descriptions rather than as reality"--a stance that may be necessary for individuals to generate new and potentially more empowering metaphoric frames (Schon, 1993, p. 160). With such a 
stance, the boundaries of what reality is, what is thinkable, knowable and doable become more permeable. This kind of permeability in LEC is extended to include what is possible between people, allowing for the experience of connection and seeing one's self as embedded in an expressive community (Katz, 1984). The central importance of conversation as an activity that creates realities and maintains them through processes of agreement in LEC (i.e. "interim reality"; see pp. 93-94) makes community a potentially important concept. One's "self" is also viewed as a social construction and as such, who one is, and to a certain extent what is possible, is shaped by the communities one participates in.

One familiar piece of conversation that takes place in the Advanced Course is that human beings tend to view themselves as separate and alone, which leaves them feeling isolated, powerless, defensive, cynical, etc. In actuality, it is said, human beings are more like the fingers on a hand: while each finger is distinct, it is not really separate or isolated from the rest of the fingers. Lerner (1986) claims that as people begin to experience themselves as profoundly connected rather than isolated, this creates a form of transformation and the possibility of seeing oneself as powerful.

While the metaphors abstracted from LEC's metaphoric system for this study do not explicitly structure an expression or experience of community among those who participate, this kind of connection is consistently reported by those who participate in LEC courses: 
[I]t was really stunning my--quite frankly my first inclination when I went there was who are these bozos? And then by the end of the time I'm going these are-these bozos are about 150 of the most sharing people, I mean it was just stunning what these people would get up and say. . . Almost anyone stands up and there's some little thing that can resonate with yourself. (Carl, 452-59)

The most memorable impression I got from it, the biggest impact it had on my life was just the connection with other human beings. . . Just the unbelievable humanity of people, paying attention to other people and really listening to what they said and that kind of--how many people did that, 150 people? And just feeling connected with all of them all at once and it was just, that almost overwhelming it was so cool. You know, and that was, I've never had anything like that. (Mora, 147-156)

What ever else LEC is or does, the implications point to a community and a culture where members are empowered through a focus on "living as a conversation," and an experience of transformation in the way that they interpret and approach the world they live in:

$\square$ Well, what Landmark has given me is--it really is a powerful way of living. And to really know that I own that power, and I can take it anywhere I want to and I am responsible. (Leigh, 1193-96)

I guess overall its powerful because it opens up all these new choices and possibilities that weren't there. They couldn't exist in the paradigm you lived in before. They wouldn't exist. (Jenna, 668-67)

LEC's community also seems to effectively fulfill the function of a "plausibility structure"--a necessary social condition for "resocialization": the process of transforming and then maintaining a social reality:

The most important social condition is the availability of an effective plausibility structure, that is, a social base serving as the 'laboratory' of transformation. This plausibility structure will be mediated to the individual by means of significant others with whom he [or she] must develop strong affective ties. ... Alternation is predicated on a reorganization of "the conversational apparatus. 
The partners in significant conversation change. And in conversation with new significant others subjective reality is transformed. It is maintained by continuing conversation with them, or within the community they represent.

(Berger \& Luckmann, 1966, pp. 157;159)

Thus, the experience of and desire to participate in the community associated with LEC's courses is an important criterion for creating and sustaining transformation and empowerment by "institutionalizing" and "sedimenting" LEC's metaphoric system and worldview as a social reality. With these things in mind, LEC's community can be seen as part of the process of transformation and empowerment, as well as a product of those experiences.

\section{Unanticipated Findings}

Chapter four included a number of examples suggesting the ways in which LEC cultural members make use of and understand the metaphoric system and experiential structure associated with LEC. I would like to address a slightly different but related question here, that while not posed as one of the research questions for this study, is relevant to the issue of individual's lived experiences: how do individuals adapt to or incorporate a new or different metaphorical structuring into their existing structure (i.e. conceptual system)?

An interesting observation was made late in analysis that suggests a process of coordination between LEC's meaning structures and those meaning structures already part of the individual's conceptual system. In four of the five interviews I observed that each participant demonstrated a pattern of metaphoric usage that 
seemed to be a reflection of their unique position and experiences in life. These individual preferred frames were used to share their understanding of and experiences with LEC's meaning structures.

For example, Simon, who drives a delivery route for a major shipping and package service, used an abundance of journey and travel metaphors to talk about his experiences in LEC. His use of these journey metaphors was unusually extensive (far exceeding any journey references in the other interviews) which suggests his particular way of framing lived experience finds expression in his familiarity with journeys:

$\square$... I think that you could really make some progress in there because they, they have already--they're there, they've come with their friends or family to the Forum, or a special evening in the Forum, and they're kinda like half way there. $(165-171)$

- I don't think you can coast at all. You must become coachable, or you don't--if you wanta get it. If you want to get it. (510-12)

I mean if you can unload stuff like that in your life, I mean, just in your own mind you can just unload so much baggage. . . because people carry that stuff around, for years, for ever, for their lives. (568-576)

$\square$... you've gotta get past that, before you can move on, and once you really become at peace with yourself that's when you can really start to move forward. (580-83)

$\square$ I'm gonna kinda like go off on an exit here and talk about the Advanced course. ... I guess probably the second day when we were talking about reality and the nature of reality, when my mind was just fffoggy and I was kinda lost. . . and there were other people that were continuing the inquiry ... I I thanked those people because when I was at my weak point they carried on (662-72) 
These are only a few examples of Simon's use of journey metaphors to share his experiences with me. Leigh, on the other hand, talked a great deal about her experiences as a mother during the interview and shared many of her insights and experiences with LEC using detailed parenting analogies and descriptions that suggested food and nourishment metaphors. For example, Leigh was sharing an insight she had about how her communication with her daughters actually created a reality for them. Leigh is not talking about food when she references "feeding"; but how and what she conveyed to her daughters through her communication:

$\square$ Because I hadn't told her I already had the confidence in her where she was going to be able to do that. And she's 7, so she seems to need, that feeding. You know, she needs to have her daily feeding, that, I'm for her. You know, that, I believe in her. . . But then, once I just started feeding her what I wanted her to $b e$. . . because I $d o$ believe, she can be that. What happened before is I expected it. And what I'm doing now is I'm letting her know, that I believe she can $b e$ it. . . I just never realized, the things that I was feeding them. . (Leigh, 832-59)

Leigh used many examples from her experiences as a parent to illustrate various insights she gained from the Forum. Mora, a computer programmer, actually pointed out that she was using a computer programming word to describe something about the Forum:

Mora: I'd also use the world integration. That's another word I would also use. . . . it seems like all the premises that I had agreed with before seemed to be presented in an integrated way. It was a way of taking views that were disparate and kinda putting them together in 3 days and talking about just that....

Me: I like that, that's a good description, too. Integration. 
Mora: Mm. A programmer (laughing) word. (lines 881-895)

Mora used the word "integration" in several places throughout the interview to describe aspects of the Forum or her experience in it. Ivan, devoted Christian, shared extensively with me about his own inquiry into how LEC's "design principles" aligned with the "spiritual design principles" of Christianity. Here are just two examples:

$\square$... that what hell is, being it a burning lake of fire or not, its just simply the logical conclusion of what it is to be a sinner. The Forum says, a life given by rackets and winning formulas, the logical conclusion of that is no possibility. No possibility. . . And I don't think there's a whole lot of possibility in hell, you know, either. (1094-1101)

$\square$ Christ said, before he died, he said that unless a grain of wheat enters the ground and dies, it cannot become a shoot. The process is a grain of wheat, enters the ground, the husk dies and falls off and a brand new life springs up. Three days later the Christian story is, that ... the stone rolled away and Christ walked out. That, that through his dying, through taking on in his self, the logical conclusion of our sins so to speak, paying the price for it, literally, and then rising ... He was reborn. . . Sheer, utter possibility. Sheer, utter possibility in the world.... When you, when you get off a racket, you're dying to that and possibility springs up. And you know, in the Forum, the last day that, you know, that life is empty and meaningless and what you get out of the Forum is you pay two hundred and ninety dollars for is nothing, you get nothing, which is possibility. (Ivan, 1112-41)

I am not suggesting these metaphoric patterns are the only ones these individuals rely on to interpret and express their experiences. Additional analysis would likely reveal that each individual has a constellation of preferred frames for conceptualizing their experiences. The point of these examples is to illustrate that the participants interviewed for this study seem to be coordinating or synchronizing 
two different metaphorical systems and experiential structures in a way that makes sense for them.

This could be an example of what Schon (1993) called "frame restructuring." Schon notes that the process of "frame restructuring"--integrating new or conflicting metaphoric frames--might actually occur in a process similar to that of making new generative metaphors. In the process of frame restructuring, the old frames are restructured to coordinate with one another, as opposed to being mapped onto one another. Schon also suggests that the cognitive work of "frame restructuring" requires that individuals draw on "features and relations" found in the concrete situation in order to construct new stories and descriptions that achieve the coordination of new or conflicting frames. The process Schon describes may actually take place during the Forum (or other LEC courses) as individuals actively work through LEC training content. Schon also notes that awareness of the frames we use to think metaphorically about experiences and the ability to restructure and generate new, potentially more effective or empowering frames, requires a stance that will allow people to "recognize descriptions as descriptions rather than as reality" (p. 160). Clearly, the key meaning structures in LEC's metaphorical system frame realities as products of human "descriptions" through language use in conversation. LEC's root-metaphors alone provide a set of inference patterns that, if adopted, would provide just such a stance. 
Another possibility is that the emphases on creation, flexibility, and selfreflexive awareness built into LEC's experiential structure allows individuals to be creative in adapting and adopting LEC's metaphors to accommodate aspects of their unique worldviews. All of LEC's metaphors provide structuring adaptable to many different individuals and situations (e.g. the idea that "life is a conversation" can be applied to any and all situations and to all human beings). Those metaphors structuring the concept of "transformation" (see pp. 174-93) occurred as especially adaptive. Sackmann's (1989) research suggested that "adaptive metaphors" were especially useful in facilitating the process of organizational transformation.

"Adaptive metaphors": "describe a transformation process whose outcome cannot be specified at the outset. They connote a continuing process of search, taking action, and adjustments toward 'fuzzy' goals which may change during the process"; adaptive metaphors "can accommodate random variation and changing conditions without becoming obsolete" (p. 471). It is possible that adaptive metaphors also facilitate the process an individual must go through to incorporate, accommodate, or integrate new metaphoric structures into their existing meaning structures.

\section{A Critical Look at LEC's Emergent System}

Any metaphorical concept or system of metaphorical concepts (or for that matter any symbolic system) privileges certain domains of experience (e.g. ways of thinking, speaking, acting and understanding), focusing our attention a particular aspect or aspects of something. At the same time, metaphorical concepts downplay 
or obscure other domains of experience that may be inconsistent with them (Lakoff \& Johnson, 1980). Metaphors provide partial structuring and thus, partial understanding of concepts.

To this point, much attention has been given to the aspects of experience that LEC's metaphoric system highlights, with less being invested in examining what this system down plays or obscures. However, some examples of what is down played by the various metaphors have been given in several earlier sections (e.g. p. 144; 166-67). The goal here is reiterate this aspect of metaphoric structuring and to touch on some of what the system as a whole seems to downplay or make it difficult to focus on.

Consider that LEC's metaphoric system essentially places responsibility for what things mean with the individual, which tends to down play the role of intentionality that may actually be behind some form of discourse or action. For example, person A might say to person B "You don't approve of the way I work" in response to person A's "feedback" about his or her work habits. Person A might then say, "I didn't say that, that's just what you made it mean" and avoid addressing the intent behind the communication or taking responsibility for one's potential impact on others. Thus, while LEC's system of meanings tends to facilitate personal responsibility, it could also be interpreted to obscure mutual responsibility in some instances. This represents an incongruity in the system, especially when one considers the following LEC premise for being a responsible communicator: "take 
responsibility for what people are left with after [your] conversations with them" (CAP notes).

LEC's metaphors highlight language and communication as creative acts that, among other things, produce various kinds of structures for human "being." What these metaphors seem to down play is that if communication can be used to create, it can also be used to destroy. It may be that in LEC's system, the negative connotations of "destroy" are encompassed within the realm of individual interpretation, again down playing the potential for and existence of malign intent. While meaning-making may be an individual product, knowledge of intent could in fact be an important consideration.

LEC's system privileges that which is newly created, not based in the past, open to invention, and not limited to what has been. Everything is a function of conversation and thus, anything can be altered or created through conversation. LEC's metaphoric system does include metaphors framing the products of creation, but process seems to be more highly prioritized as does the flexibility to move beyond any product (e.g. structure) one creates. This focus may tend to obscure the potentially negative impact of impermanence. That is, in valuing the new and not yet existing, the values of the past and the benefits of stability might be overlooked. Herein also lies a strong potential for frame conflict, as the dominant mainstream culture would tend to value stability and predictability. Stability and change tend to co-exist in a sort of tension or balance, however, LEC's system seems to value 
change more than stability in some permutations--a prioritization that obscures the value of balance between the two.

As explored earlier in this chapter, the system of metaphors abstracted in this study tend to highlight the power of the individual to choose, create, alter and to a certain extent, control one's reality and experiences. While this may be the case, the metaphors emerging in this study down play the fact that social structures of power are not necessarily altered when the individual realizes his or her own power. The significance of this issue, as well as practical approaches for dealing with and altering socially constructed realities are addressed in LEC's curriculum, but metaphors for structuring these ideas did not specifically emerge.

In addition to obscuring certain aspects of experience, metaphoric systems, like other symbolic systems, possess internal and inherent inconsistencies and schisms, most notably as these systems are interpreted and enacted by human beings with different needs, goals and interests. In this case, such inconsistencies and schisms are most obvious when considering the influences on and interpretations of the system that are introduced by LEC as an organization.

One very obvious inconsistency exists in the apparent contradiction between the capitalistic goals of LEC as a for-profit organization and the service goals they adopt as an educational organization providing transformational courses to contribute possibility to the lives of human beings. While LEC's stated goals may be noble and while their educational product may even effectively deliver on those 
goals, the organization's primary goal must still be to maintain its own existence. Thus, the conflict between self-serving and community-serving goals cannot help but remain a constant, if often subtle, influence on organizational choices and actions.

For example, one of LEC's methods for ensuring the organization's existence seems to be "piggy-backing" marketing practices onto practices that allegedly further individuals "training and development" in particular areas. The practice of "sharing the Forum" is claimed to provide an opportunity to overcome those things that "stop" one in life, such as fear of rejection or lack of confidence in being selfexpressed. "Sharing the Forum" is also a key practice for sharing one's own experience of transformation as well as transforming communities. At the same time, the organization relies heavily on "sharing the Forum" to bring new participants to their courses, and thus, maintain their existence. LEC also relies heavily on the support of volunteers to present their programs and run daily organizational operations. Serving in this way--"assisting"--is also framed as a method of providing additional training and development in various areas such as service, leadership, and accountability.

One informant reported that his awareness of this internal conflict between capitalistic and service oriented goals was the source of suspicion and cynicism regarding LEC's motives as well as the credibility of their ideas and worldview. These issues were a major factor in his decision not to further his participation, as he perceived they could not really have his best interests at heart or truly develop a 
system of ideas that would ultimately put themselves out of business if all were to "convert" (Ash, 1-18-97).

Another problematic aspect of LEC's organizational interpretations of their "design principles" and "technology" is the potential to use that system to avoid or reverse responsibility. For example, if "human beings are meaning-making machines" and events themselves (the domain of "what happened") are "empty and meaningless," then ultimately the individual is responsible for the meanings he or she makes of experience. The potential then exists for the organization to claim that a participant who had a negative experience of some kind in an LEC course "made up" those meanings and is thus, responsible for them. LEC might even suggest that the problematic issues and meanings the individual experienced represent "rackets" or "stories" or some other "conversation" that often stop them in life and could be "transformed." Indeed, LEC seems to be able to use some aspect of their own technology to deflect challenges to that technology. According to one philosopher: "You cannot debate the Forum, you just start talking its language or you don't" (Gottlieb, 1990. p. 5).

The issue of language is in fact, another current of tension in LEC's system that many find problematic. Gottlieb (1990) points to the irony of LEC's "way of speaking" when he says that in order "to open the possibilities in your own life" and "free yourself from the categories of everyday language, you have to be imprisoned in a new jargon that few other people speak" (p. 5). LEC's "applied ontology" is 
embedded in a very particular way of speaking and fully adopting LEC's meanings and practices is to a certain extent dependent upon learning that language. Of course, there are theoretical premises supporting the need for a new language to create and support a new reality, some of which have already been discussed in this chapter. The core of those theoretical premises is that we inherit a world already constituted in language, which constrains what is thinkable, knowable and doable. Major of alterations in what is possible at the level of individual or social reality would thus depend on alterations in the language constituting reality (Berger \& Luckmann, 1966; Lerner, 1986).

Whether or not participants are "imprisoned" by LEC's language, they frequently express difficulties with or reservations about what is sometimes called "Landmark-speak," "Landmark-ese," or "Forum-speak." Some, like the informant Ash, are very resistant to using LEC's language, perceiving it as an unnecessary attempt to force acceptance of LEC meanings which may not fit with the individual's view of the world. A common theme among those interviewed for this study is that while LEC's language facilitated conversation among those sharing a background in LEC, it was not effective for communicating with those who do not.

$\square$ Before I did the Forum, I could hear people, you know, talk about possibility and rackets and winning formulas, and they were all using the same words and .. it just felt kinda weird and brainwashy to me because ... I didn't know what those words meant. (Ivan 1699-1704) 
$\square$ you want to be able to speak a language that people really relate to and if you use all this kinda weird jargon, they're gonna [think]. . you've been brainwashed. (Mora, 562-68)

$\square$ Its part of what you get when you're involved in Landmark for any length of time. Ah, you get it actually from the Forum, I mean, you start using words. . . they're like buzz words. . . Its like, if I was a computer programmer, talking to another computer programmer buzz words that only computer programmers-its like an insider's language. And that's kind of the way I look at Landmark language, Landmark-speak. Ah, its kind of an insider's language (Simon, 12741361)

What might be called issues of language "integration" and "translation" are often discussed in LEC courses and participants are encouraged to speak in "plain English" (AC homework handout) to their friends and families when sharing about their LEC experiences. Translation and interpretation of LEC's concepts became critically important in this thesis in order to both analyze the data and communicate the findings. One interviewee shared her thoughts on these issues:

$\square$... if it was going to be completely integrated in my life I guess I'd have to get used to using the language or I'd have to talk about these issues in a language that makes me really comfortable. . . Be able to integrate it and use words that, that speak about these things that people can relate to. (Mora 554-580)

Some note the potential for this language use to become an "ego" display of membership or to be perhaps be used mechanically or "inauthentically":

$\square$ I was just starting to get the language, when I was doing the S.E.L.P., I mean, using it fluently. Not like crazy fluently, but I was able to drop in a word here or there . . . it was almost an ego thing, when you're doing it . . But, when I said it, I was very pleased with myself, because it said, "yeah, I've been doing this Landmark stuff for awhile now. I can talk like this, I can use this word." And, its interesting, I think that people get caught up in the language (Simon, 1277-87) 
there's times when I've just been really tired hearing somebody share, in just complete perfect Landmarkese. . . . when I hear somebody talking in that specific way it just feels like there's a machine going on--and its just my interpretation. . . . But also I think that--I guess this is more of a question: does that specific accent, the Landmark accent, have a--is it something that can be a barrier for people, to authenticity, that they can hide behind the Landmark accent and not be real about what they're saying and what they're going through? ... . I think a person can use Landmarkese with the accent and the words, and if they're authentic about it, you can have a powerful interaction with them. (Ivan, 1750-1851)

One source of tension for interviewees is that in spite of these concerns and criticisms, they can also see the potential usefulness or value in the particular way of speaking:

$\square$ But, its like with any group of thought, its language that distinguishes it from another language and that specific language gives one possible way of being when you take it on and you commit yourself to it. . . And, I can see that using that vocabulary gives you access to distinctions in your life (Ivan 1845-55)

All of the interviewees demonstrated a heightened awareness of the relationship between language and reality and that LEC's language is thus, instrumental to creating the view of reality that LEC constructs.

The internal inconsistencies and schisms present in LEC's system does not necessarily negate its value or diminish the possibility and empowerment many people derive from it. Similarly, the existence of these issues does not indicate intent on the part of LEC craftily use its power to achieve its own goals at the expense of those it claims to serve. Such conflicts of interests and issues of power are unavoidable in the process of producing and reproducing social and organizational realities (Mumby, 1988). My own goal in highlighting some of the inconsistencies 
arising in the interpretation and enactment of LEC's metaphoric system is to foster critical thinking and healthy skepticism when considering what is, after all, an ideological discourse. No system of ideas, no matter how enticing, should be taken at face value and no system of ideas has "all the answers," regardless of what its proponents may say. For my own part, I find much that is valuable in LEC's approach to living and have seen their courses make a remarkable difference in the lives of so many. In order for LEC to continue making a difference, I believe people must continue to interact critically with the ideas and the organization.

\section{Limitations of this Study}

Several limitations in this study should be acknowledged. One limitation was incomplete access to The Landmark Forum as a "text" for analysis. The Forum is the beginning of "the conversation," the place where the structure and logic of this culture's metaphoric system is initially developed. Because LEC does not allow note-taking during the Forum, my access to these initial meaning structures was through interviews, my own experiences, and through course notes which reviewed or further developed initial meaning structures. Similarly, a lack of access to individuals and texts more directly reflecting the organization's culture means that the larger influence of the organization on the symbolic system is not adequately represented here.

My familiarity with and membership in LEC's cultural context represents both a strength and a limitation. The detailed explication of LEC's meaning structures 
would not have been possible without my "insider" status. However, closeness to the topic may have constrained my ability to "see the forest" of the larger metaphoric structures while wandering amidst "the trees."

Underestimating the complexity of LEC's metaphoric system resulted in a less detailed metaphor analysis than originally intended. A more extensive analysis including metaphoric mapping of ontological correspondences would more accurately reflect the systematicity present in this metaphoric system.

The sample in this study was carefully selected to provide diversity in terms of participants' background, as well as, the length (number of years) and of depth (number of courses) of their participation with LEC. However, a larger sample would have better represented the natural diversity present in LEC's community.

Finally, metaphor analysis allowed for the description of meaning structures, but the design of this study did not accommodate examining the aspect of process. Transformation whatever else it may be, is not static. Understanding the experiential process through which LEC's metaphoric system is delivered and developed would likely reveal more about participants' experience of transformation, empowerment and community than could be addressed in this study.

\section{Implications for Future Research}

The results of this study indicate that further research is needed to understand the process by which individuals adopt or adapt new metaphoric meanings into their existing conceptual framework, as well as the conditions and factors that seem to 
facilitate or interfere with that process. The findings in this study also suggest that there is a relationship between becoming an active member of a culture and the adoption of or adaption to that culture's metaphors, which is congruent with Deetz (1984) position. More metaphor analysis in cross-cultural contexts could provide useful information about the processes of acculturation. However, this study also revealed that current analytic frames for metaphor analysis (with few exceptions, e.g. Deetz, 1984) do not adequately address issues of conducting and translating cross-cultural metaphor analyses. Future research might also investigate the systems of metaphoric meaning structures present in other transformational "conversations" such as neurolinguistic programming (NLP) and logotherapy in order to explore their similarities and differences (e.g. Are there meaning structures common across processes of transformation?).

\section{Conclusion}

As one interview commented: "Transformation is just a word . . the thing is, we need a language to talk about what Landmark does, and what it is" (Simon, $1198 ; 1234-35)$. The goal of this study was to investigate the concept of "transformation" by taking a closer look at the language used in this community to talk about what Landmark is and what it does through the lens of metaphor analysis. Emerging from this analysis is evidence of a unique system of metaphors associated with an alteration or shift in participants' perceptions and enactments of communication, their conceptualizations of reality, and their experience of 
themselves and others in fundamental ways. The worldview that these metaphors privilege is in many ways an alternation of the conventionally held views and values of mainstream U.S. culture. The important question we are left with is not whether or not these metaphors are "true" propositions about the world, but what sort of world do they propose? We would do well to ask this of our own taken-for-granted metaphors, personally, socially, and globally.

Language is the bridge between the uncreated and created worlds. It is both the ultimate reality and the instrument through which reality is brought forth. --Parabola 


\section{REFERENCES}

Axley, S.R. (1984). Managerial and organizational communication in terms of the conduit metaphor. Academy of Management Review, 9 (1), 428-437.

Babbie, E. (1992). The practice of social research (6th ed). Belmont: Wadsworth.

Bauman, R. \& Sherzer, J. (Eds), (1974). Explorations in the ethnography of speaking.

Berger P.L. \& Luckmann, T. (1966). The social construction of reality: A treatise in the sociology of knowledge. Anchor: New York.

Black, M. (1962). Metaphor. In M. Black, Models and metaphor. (pp. 25-47). Ithaca, NY: Cornell University Press.

Black, M. (1993). More about metaphor. In A. Ortony (Ed.), Metaphor and thought. (pp.19-41). Cambridge: Cambridge University Press.

Braithwaite, C.D. (1991). Cultural communication among Vietnam veterans: Ritual, myth and social drama. In R. Morris \& P. Ehrenhaus (Eds.), Cultural legacies of Vietnam: Uses of the past and present. Norwood, NJ: Ablex.

Brownell (May, 1996). The theorectical base of gestalt psychotherapy. $<$ http://www.europa.com/ brownell/theory/html > (1996, May 5).

Bulmer, M. (1979). Concepts in the analysis of qualitative data. Sociological Review, 27,(4), 651-677).

Deetz, S. (1984). Metaphor analysis. In William B. Gudykunst \& Young Yun Kim (Eds.), Methods for Intercultural Communication Research (pp. 215-228). International and Intercultural Annual, v. 8. Sage: Beverly Hills.

Ely, M., Anzul, M., Friedman, T., Garner, D., \& McCormack Steinmetz, A. (1991). Doing qualitative research: Circles within circles. London: Falmer Press.

Foss, S.K. (1989). Rhetorical criticism: Exploration \& practice. Prospect Heights: Waveland.

Freeman, E., \& Appel, D. (1970). The wisdom and ideas of Plato. Greenwich: Fawcett. 
Gottlieb, A. (1990, January 7). Heidegger for fun and profit. The New York Times, Sunday late edition, sec. 7, p. 1, col. 1. (reprinted with permission on the internet: http://www.inlink.com/ dhchase/heidig.htm)

Guba, E.G. \& Lincoln, Y.S. (1994). Competing paradigms in qualitative research. In N. Denzin \& Y. Lincoln (Eds.), Handbook of Qualitative Research. Newbury Park: Sage.

Honigman, J.J. (1970). Sampling in ethnographic work. In R. Naroll and R. Cohen (Eds.), A Handbook of method in cultural anthropology (pp. 266-281). Garden City: The Natural History Press.

Hymes, D.H. (1962). The ethnography of speaking. In T. Gladwin \& W. Sturtevant (Eds.), Anthropology and Human Behavior, (pp. 13-53). Washington, D.C.: The Anthropological Society of Washington.

Hymes, D.H. (1972). Toward a descriptive theory. In J. Gumperz \& D. Hymes (Eds.), Directions in Sociolinguistics: The ethnography of Communication, (pp. 52-71). Holt, Rhinehart \& Winston.

Hymes, D.H. (1974). Foundations in sociolinguistics. Philadelphia: University of Philadelphia Press.

Jackson, S. (1996, April 18-24). When it comes to Landmark Education Corporation, there's no meeting of the minds. Westword.

Kallick, D. (1989). The speakerly teacher: Socrates and writing. Metaphilosophy, 20 , (3 \& 4), 341-346.

Katz, R. (1984). Empowerment and synergy: Expanding the community's healing resources. In J. Rappaport, \& R. Hess (Eds.), Studies in empowerment: Steps toward understanding and Action (pp.201-226). New York: Hawthorn.

Kieffer, C. (1984). Citizen empowerment: A developmental process. In J. Rappaport, \& R. Hess (Eds.), Studies in empowerment: Steps toward understanding and Action (pp. 9-36). New York: Hawthorn.

Kirk, J. \& Miller, M.L. (1986). Reliability and validity in qualitative research. Newbury Park: Sage. 
Koch, S. \& Deetz, S. (1981). Metaphor analysis of social reality in organizations. Journal of Applied Communication Research, 9 (1), 1-15.

Lakoff, G. (1993). Contemporary theory of metaphor. In A. Ortony (Ed.), Metaphor and thought.(pp. 202-251). Cambridge: Cambridge University Press.

Lakoff, G. \& Johnson, M. (1980). Metaphors we live by. Chicago: University of Chicago Press.

Langan, T. (1961). The meaning of Heidegger: A critical study of an existentialist phenomenology. New York: Columbia University Press.

Landmark Education Corporation Homepage. (July 1997). <http://www.landmarkeducation.com/forum/home.htm>

LeCompte, M.D. \& Goetz, J.P. (1982). Problems of reliability and validity in ethnographic research. Review of Educational Research, $\underline{52}$ (1), 31-60.

Lemay, E. \& Pitts, J.A. (1994). Heidegger for beginners. New York: Writers and Readers Publishing.

Lerner, M. (1986). Surplus powerlessness: The psychodynamics of everyday life... and the psychology of individual and social transformation. New Jersey: Humanities Press International.

Lincoln, Y.S. \& Guba, E.G. (1985). Naturalistic inquiry. Newbury Park: Sage.

Littlejohn, S.W. (1992). Theories of human communication. (4th ed). Belmont: Wadsworth.

Lofland, J. \& Lofland, L.H. (1995) Analyzing social settings: A guide to qualitative observation and analysis (3rd ed). Belmont: Wadsworth.

McCracken, G. (1988). The long interview. Newbury Park: Sage.

Marshall, C. \& Rossman, G.B. (1995). Designing Qualitative Research. (2nd ed.). Thousand Oaks: Sage.

Morgan, G. (1980). Paradigms, metaphors, and puzzle-solving in organizational theory. Administrative Science Quarterly, 25(4), (pp. 605-622). 
Mulhall, S. (1996). Routledge philosophy guidebook to: Heidegger and being and time. London: Routledge.

Mumby, D.K. (1988). Communication and power in organizations: discourse, ideology and domination. Norwood, N.J.: Ablex.

Ortony, A. (1993). Introduction to metaphor and thought. In A. Ortony (Ed.), Metaphor and thought. (pp. 1-27). Cambridge: Cambridge University Press.

Palmer, D. (1995). Sarte for beginners. New York: Writers and Readers Publishing.

Patton, M. (1996). Qualitative evaluation and research methods (2nd ed.). Newbury Park: Sage.

Philipsen, G. (1977). Linearity of research design in ethnographic studies of speaking. Communication Quarterly, 25 (3), 42-50).

Philipsen, G. (1992). Speaking culturally: Explorations in social communication. Albany: State University of New York Press.

Reddy, M.J. (1993). The conduit metaphor: A case of frame conflict in our language about language. In A. Ortony (Ed.), Metaphor and thought.(pp. 164-201). Cambridge: Cambridge University Press.

Richards, I.A. (1936). The philosophy of rhetoric. New York: Oxford University Press.

Rappaport, J. (1981). In praise of paradox: A social policy of empowerment over prevention. American Journal of Community Psychology, 9 (1), 1-25.

Rappaport, J. (1984). Studies in empowerment: Introduction to the issue. In J. Rappaport, \& R. Hess (Eds.), Studies in empowerment: Steps toward understanding and Action (pp. 1-7) New York: Hawthorn.

Ricoeur, P. (1976). Interpretation theory: Discourse and the surplus of meaning. Fort Worth: Texas University Press.

Riessman, C.K. (1993). Narrative Analysis. Qualitative Research Methods V.30. Newbury Park: Sage. 
Rohrer, T. (1995, Summer). Conceptual blending on the information highway: How do metaphorical inferences work? Paper presented at the International Cognitive Linguistics Association, Amersterdam, Johns Benjamin.

Sackmann, S. (1989). The role of metaphor in organizational transformation. Human Relations, 42(6), 463-485.

Saville-Troike, M. (1982). The ethnography of communication: An introduction. Basil Blackwell.

Schatzman, L. \& Strauss, A.L. (1973). Field research: Strategies for a natural sociology. Englewood Cliffs, NJ: Prentice Hall.

Schulte, J. (1992). Wittgenstein: An introduction. (W. H. Brenner \& J.G. Holley, Trans.). Albany: State University of New York Press.

Schon, D. (1993). Frame awareness, frame restructuring and frame conflict: A generative theory of metaphor. In A. Ortony (Ed.), Metaphor and thought.(pp. 137-163). Cambridge: Cambridge University Press.

Self, J. (1992). 60 minutes and the assassination of Werner Erhard. Houston: Breakthru Publishing.

Smith, R.C. \& Eisenberg, E.M. (1987). Conflict and Disneyland: A root-metaphor analysis. Communication Monographs, $\underline{54}, 22-35$.

Spradley, J.P. (1979). The ethnographic interview. Orlando, FL: Holt, Rhinehart, \& Winston.

Stewart, J. \& Philipsen, G. (1984). Communication as situated accomplishment: The cases of hermeneutics and ethnography. In B. Dervin \& M.J. Voigt, (Eds.), Progress in Communication Sciences (Vol. V, pp. 177-217). Norwood, N.J.: Ablex.

Sticht, T.G. (1993). Educational uses of metaphor. In A. Ortony (Ed.), Metaphor and thought. (pp. 621-632). Cambridge: Cambridge University Press.

Strauss, A.L. \& Corbin, J. (1990). Basics of qualitative research: Grounded theory procedures and techniques. Newbury Park: Sage. 
Thompson, J.B. (Ed.) (1981). Paul Ricoeur: Hermeneutics and the human sciences. Cambridge: Cambridge University Press.

Welkos, R.W. (1991, December 29). Scientologists ran campaign to discredit Erhard, detective says. The Los Angeles Times, sec. A, p. 1; col.1.

Whorf, B. L. (1956). Language, thought and reality. New York: John Wiley.

Yontef, G. (1993). Awareness, dialogue, and process. The Gestalt Journal Press. Highland, NY. 


\section{APPENDIX A}

\section{LEC PROPOSAL LETTER}

July 18,1996

Harry Rosenberg, Chief Executive Officer

c/o Christina Anderson, Executive Assistant

Landmark Education Corporation

899 Howard Street, Suite 501

San Francisco, CA 94103

Greetings!

Per my telephone conversation with Christina, this letter of introduction will be followed by a brief proposal describing my master's thesis research project, which I would like to conduct with the cooperation of Landmark Education Corporation (hereafter referred to as LEC). Before going into the actual proposal I would like to tell you a little about who I am and how I came to be interested in the proposed research project.

My scholarly interest in LEC grew out of my personal experiences as a active participant in the various courses and seminars. I became involved with LEC during a period of my life where I was feeling "stuck," like there was something more to life--and my life in particular--that I did not know how to reach or uncover. I was not unhappy really, just not fully engaged; feeling a lack of power and purpose in my life. I did the Forum in November of 1994 and have since completed the Curriculum for Living, the Communication: Access to Power course, three graduate seminars, and am scheduled to be a coach for an upcoming SELP. My participation in LEC's courses continues to have a profound impact on my life, manifesting in extraordinary accomplishments and an ongoing commitment to my own and others' training and development. One of those unpredictable accomplishments is the fact that I am within less than a year of completing my masters degree in the field of communication. I had given up on ever completing my graduate work and after distinguishing the stories I had built around that area of my life, I dramatically altered my life to reenter the conversation called "masters thesis."

What I have experienced and observed in others during my association with LEC is dramatic shifts in the way people live their lives in all areas. The key to the manifestation of these shifts seems to dwell primarily in communication--developing a new awareness of how one communicates and taking action through 
communication to consciously create, to live intentionally, rather than at the effect of their circumstances. This is an incredibly exciting observation for a scholar and instructor of communication! What I have observed about LEC's technology in producing such remarkable results is, among other things, the use of metaphor to initiate a process of transforming the human conceptual system, and hence, what is real. My research is informed by an approach to metaphor that elevates it in significance beyond the domain of artful speech to the domain of how we are designed as human beings.

Lakoff and Johnson (1980), in their book Metaphors We Live By ${ }^{1}$, build a compelling argument to support their claim that the conceptual system giving rise to human thought and action, the conceptual system defining the everyday realities we experience, is "fundamentally metaphorical in nature. . . human thought processes are largely metaphorical. This is what we mean when we say that the human conceptual system is metaphorically structured and defined." (p. 3,6). In order to study and better understand the nature of human conceptual systems, Lakoff and Johnson look to language:

Since communication is based on the same conceptual system that we use in thinking and acting, language is an important source of evidence for what that system is like. . . Metaphors as linguistic expressions are possible precisely because there are metaphors in a person's conceptual system... . Since metaphorical expressions in our language are tied to metaphorical concepts in a systematic way, we can use metaphorical linguistic expressions to study the metaphorical nature of our activities. (pp. 3, 6-7)

Metaphors embedded in our ways of speaking are expressions of metaphorically structured concepts that at least partially constitute our mindscape, our software for living. The implication is that metaphor is much more than a linguistic device. Metaphor not only describes our world in particular ways, it is the particular way we experience our world:

In all aspects of life. . . we define our reality in terms of metaphors and then proceed to act on the basis of the metaphors. We draw inferences, set goals, make commitments, execute plans all on the basis of how we in part structure our experience, consciously and unconsciously, by means of metaphor (Lakoff \& Johnson, 1980, p. 158)

The relationship between conceptual systems, metaphor, thought, and action suggests that significant changes in the way an individual thinks and behaves are accompanied by changes in an individual's conceptual system, and thus, by implication, changes in the metaphors they live by. If indeed metaphorical concepts infuse the very fabric of human thought and action, then it follows that the 
introduction and integration of a new metaphor or system of metaphors into one's conceptual system could have the power to redefine one's reality, and hence, the nature of thought and action. In their discussion of the relationship between new metaphors, action and reality, suggest that:

New metaphors have the power to create a new reality. This begins to happen when we start to comprehend our experience in terms of a metaphor, and it becomes a deeper reality when we begin to act in terms of it. If a new metaphor enters the conceptual system that we base our actions on, it will alter that conceptual system and the perceptions and actions that the system gives rise to. (p. 145)

So, for example, when people begin to participate in life from the new metaphor LIFE IS A CONVERSATION, they experience remarkable shifts--transformation-that have a profound impact on how they see and act in the world. The purpose of my study is to investigate the potentially transformative and empowering impact of new metaphors by exploring the system of metaphors occurring within the community of people who participate with LEC in a variety of ways (e.g. taking courses, being on staff, assisting). I would like to use Lakoff and Johnson's framework to study the metaphors occurring in the speaking and informing the actions of the LEC community of speakers.

Based on the above, the general questions that will guide my research are as follows:

1. What constitutes the system of metaphors embedded in and abstractable from various LEC originated or associated texts? ("texts" being analyzable examples of communication such as organizational literature, transcribed interviews, observation notes, etc.)

2. What does the system of metaphors highlight and downplay about human experience?

3. How might this system of metaphorical concepts give shape to and be evidenced in the everyday experience of those who participate in this system?

Collecting the metaphors--the data--for this study will be accomplished, with the approval of LEC, in several ways. One form of data collection would involve me regularly spending time at the Center doing "participant observation." I envision my role as that of an active participant at the Center, actually performing volunteer work of some kind. This would allow me to experience and observe the ways in which the metaphors specific to LEC actually get expressed in an organizational 
setting. Being an active participant would also allow me to contribute to Landmark by helping further the fulfillment of its commitments. Of course, performing this role at the Center would additionally provide an opportunity for me to receive personal training and development.

Another major source of metaphors would be individual and possibly group interviews with individuals who have been involved with LEC in various ways: participated in courses, assisted, taught seminars, worked on staff, and/or lead the Forum. An individual's participation in any interview would be voluntary and scheduled at a time and location convenient for them. I intend to enroll the participation of others as an independent student researcher, not a representative of LEC. My network of relationships within LEC is such that I see no difficulty in locating willing individuals, however, I do need your approval specifically to interview any staff member or other individual in a formal agreement with LEC.

The identity of individuals participating in the research would be protected as confidential with no actual names or identifying information appearing in tape transcripts or the final write up. I am proposing five (5) or six (6) individual interviews, each taking approximately 60 to 90 minutes, and two (2) focus group interviews from which I record and analyze participants' interactions and language use. The data collection portions of this study will take approximately three months and will begin in late August or early September. Naturally, I am eager to hear your comments, concerns, questions, and ideas regarding this research.

The study I propose is significant in several ways. First, there is a recognized need in the scientific community for studies that contribute to our understanding of the human conceptual system and the processes affecting successful change. I believe that through an analysis of the rich and compelling system of metaphors expressed within the community emanating from LEC and the trainings it offers, this study will provide such a contribution.

Second, because LEC's training content and practices appear to introduce new metaphorical concepts in order to facilitate change, transformation, or empowerment, this study will provide new insight into the relationship between metaphor, empowerment, as well as into the processes facilitating the experience of empowerment. This study will not attempt to assess that the setting represented by LEC is itself empowering by comparing it to other settings, but will instead investigate this as a case study of individuals participating in and with a particular organization whose goals and methods seem to facilitate the experience of empowerment. 
A third potential contribution lies in the area of education and training. Because the majority of what LEC provides training in is communication, I see this study as an opportunity to inquire into how metaphor might be used in the process of teaching generally and teaching communication skills specifically. If our conceptual system is indeed metaphoric in nature, then it follows that we could benefit from a better understanding of how metaphor influences various types of education and processes of learning. Also along these lines, I am a stand that my research be an opportunity for sharing this work with the academic community.

Finally, I am committed to my study benefiting LEC. Knowing that LEC is an organization committed to ongoingly developing itself and the service it provides to others, I intend that my research contribute to that goal. My observations clearly indicate that LEC exists within a model of the world that privileges the centrality of language and communication in creating and maintaining the realities we experience. The goal of my study is to look at a specific piece of that model--metaphor--and inquire into its unique power within the context of transformation and living a transformed life. This would seem to be a goal LEC would find congruent with its own.

Well, if I haven't yet overwhelmed or confused you, I hope I have convinced you that there is merit in my proposal worthy of LEC's consideration. In summary, I would like your approval to participate and observe at the Center and to interview staff and volunteers, as part of my master's thesis inquiry into the significance of metaphor as both a constitutive and expressive element of human being.

As you consider my proposal please keep in mind that a proposal is by definition one possible way to proceed. Please feel free to contact me if anything is missing, unclear or requires a conversation for alignment on some aspect. I have included an extra copy of this document for your convenience in forwarding it to another interested and/or acccountable individual. I look forward to hearing from you at your earliest convenience.

Peace and Prosperity,

Debbie Gabbert

Lakoff, G. \& Johnson, M. (1980). Metaphors we live by. Chicago: University of Chicago Press. 


\section{APPENDIX B}

\section{ACCESS AGREEMENT}

August 27, 1996

Harry Rosenberg, Chief Executive Officer

Landmark Education Corporation

899 Howard Street, Suite 501

San Francisco, CA 94103

Hello again!

In addition to extending acknowledgement and appreciation, I also want to take a moment to recreate my understanding of the major points of our conversation that relate to the conduct of my thesis research. Please let me know if my understanding is inaccurate or in need of amendment in any way. I certainly do not want to take up more of your time, but it is important to me that I proceed with as much alignment as possible. Thus, I am taking responsibility for my listening and checking it with you.

My general understanding is that while Landmark cannot at this time "be in partnership" with me in the process of this research, they also will not disapprove or attempt to hinder this project so long I adhere to the conditions agreed upon during our conversation. The following statements capture my understanding of the agreement between Landmark Education Corporation and myself regarding my research as negotiated through its representative Harry Rosenberg:

$\square$ Landmark Education Corporation does not support, endorse, or acknowledge any connection to the thesis research conducted by me (Debbie Gabbert). I will clearly establish this fact with any who choose to participate in her research project and with those who will become "consumers" of the written report (e.g. thesis committee, reviewers, colleagues).

$\square$ Landmark does not grant permission for me to interview paid staff members of Landmark Education.

- I may interview those persons in my personal network of relationships who are graduates of Landmark Education courses. I may also interview individuals who are in an assisting agreement with Landmark provided those individuals are in personal relationship with me in addition to being known to each other through 
assisting.

Landmark acknowledges that I am currently in an assisting agreement with Landmark which includes working on the CIS team at the Center and serving as a coach for the upcoming SELP (beginning Sept. 7, 1996). These activities are an expression of my commitment to the work being conducted in and through Landmark (I would be assisting in these ways whether or not I was doing research). While I am not engaging in these activities for the purpose of research, I may make observations during the course of assisting that inform my research. Should such observations be included in the research data, I will protect the confidentiality of any persons involved by using pseudonyms and leaving out identifying details.

If at any time a question arises regarding some aspect of data collection or inclusion of data, I will may contact Harry Rosenberg to clear the issue or get the question answered.

While thinking about our conversation over the last several days, it occurred to me that the process and product of my thesis research may truly not have been clear in the original proposal. I realize now that in describing my research I used what are to me common terms in qualitative research, like "interview," "observation," or even the word "research" for that matter. I apologize for assuming so much. In order to clarify what I mean when I say "interview," I have included a copy of my interview questions. Interviews are a common tool of qualitative research, which typically strives to learn about an individual's experience and meanings regarding some situation, event, process, etc. Qualitative research does not attempt to prove or expose cause and effect relationships, it attempts to explore the rich background of human experience.

In further effort to create a sense of trust in me and the conduct of this research project, I am providing you with a copy of the Human Subjects Research Review Committee (HSRRC) policies that all research in our institution must comply with in order to be approved by this committee. Each researcher must submit a detailed application to the committee that specifically outlines the data collection procedures (how interviews will be conducted, interview questions, how individuals will be informed of one's researcher status, etc.) involved in the research. The researcher must explain thoroughly how he or she will meet all of the HSRRC's policies and guidelines, such as protecting confidentiality and obtaining informed consent. If the committee is dissatisfied with the researcher's attention to the policies or if they believe the research poses too great a risk to the potential participants, the board will deny the privilege to conduct research. I thought you might find this information 
interesting and, perhaps, useful in adding to your understanding of the context in which I operate as a student researcher. I realize, of course, that these are still just words on a page and that the real issue is the integrity of the individual.

In summary Harry, I provide these explanations and information in an effort to create mutual understanding and peace of mind. I am not attempting to persuade you to alter the agreements we discussed on the phone. My goal is being responsible for my communication and creating as much shared meaning as possible. Please feel free to contact me with any further questions, concerns, comments, or requests regarding this research project. Knowing (or at least having a pretty good idea of) how busy the CEO of Landmark Education must be, I again acknowledge the time you have already afforded me in reading through correspondence and being with me on the telephone. Your commitment to Landmark and to those who consider themselves a part of this community has not gone unnoticed. Thank you!

Graciously yours,

Debbie Gabbert 


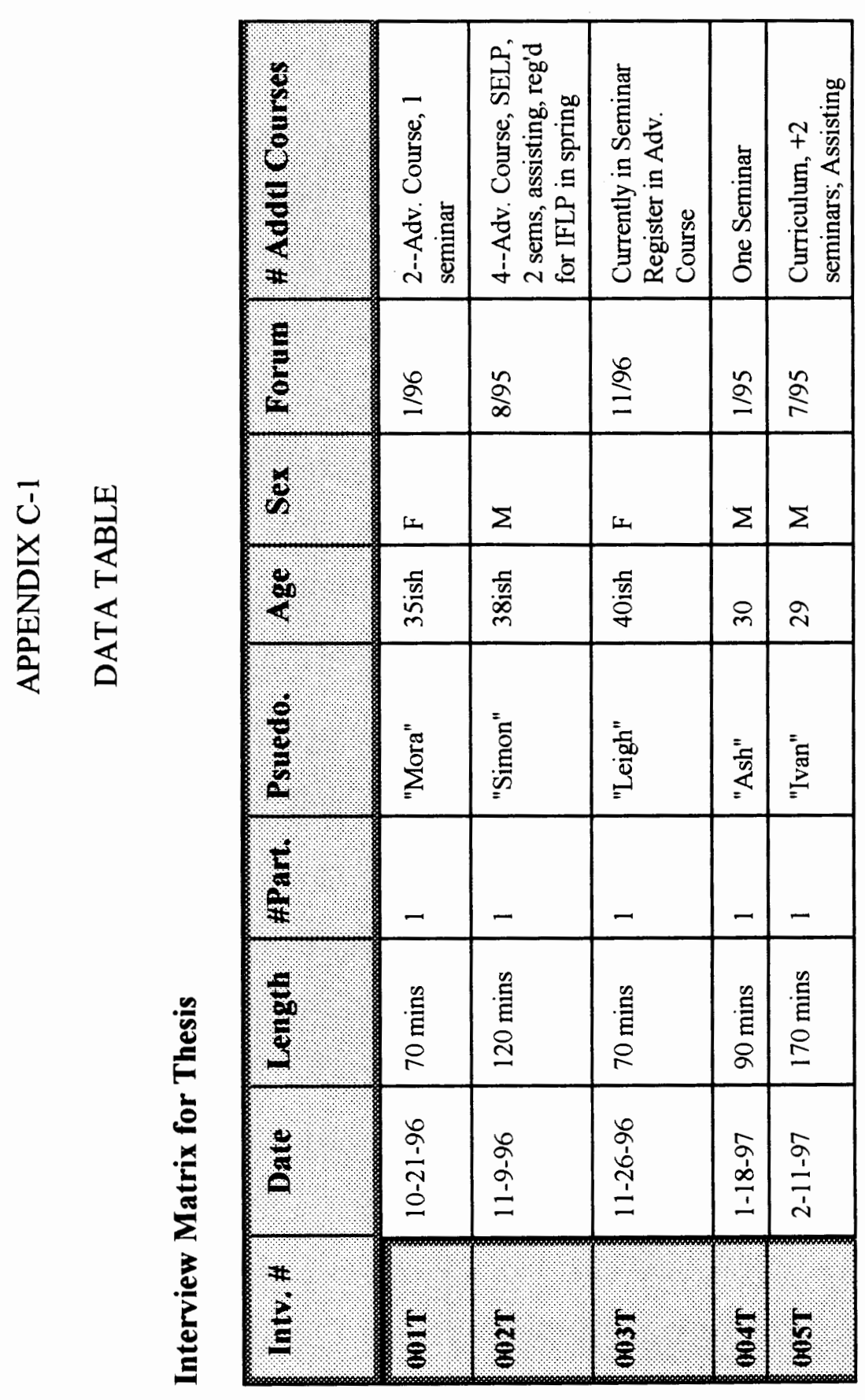

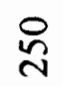




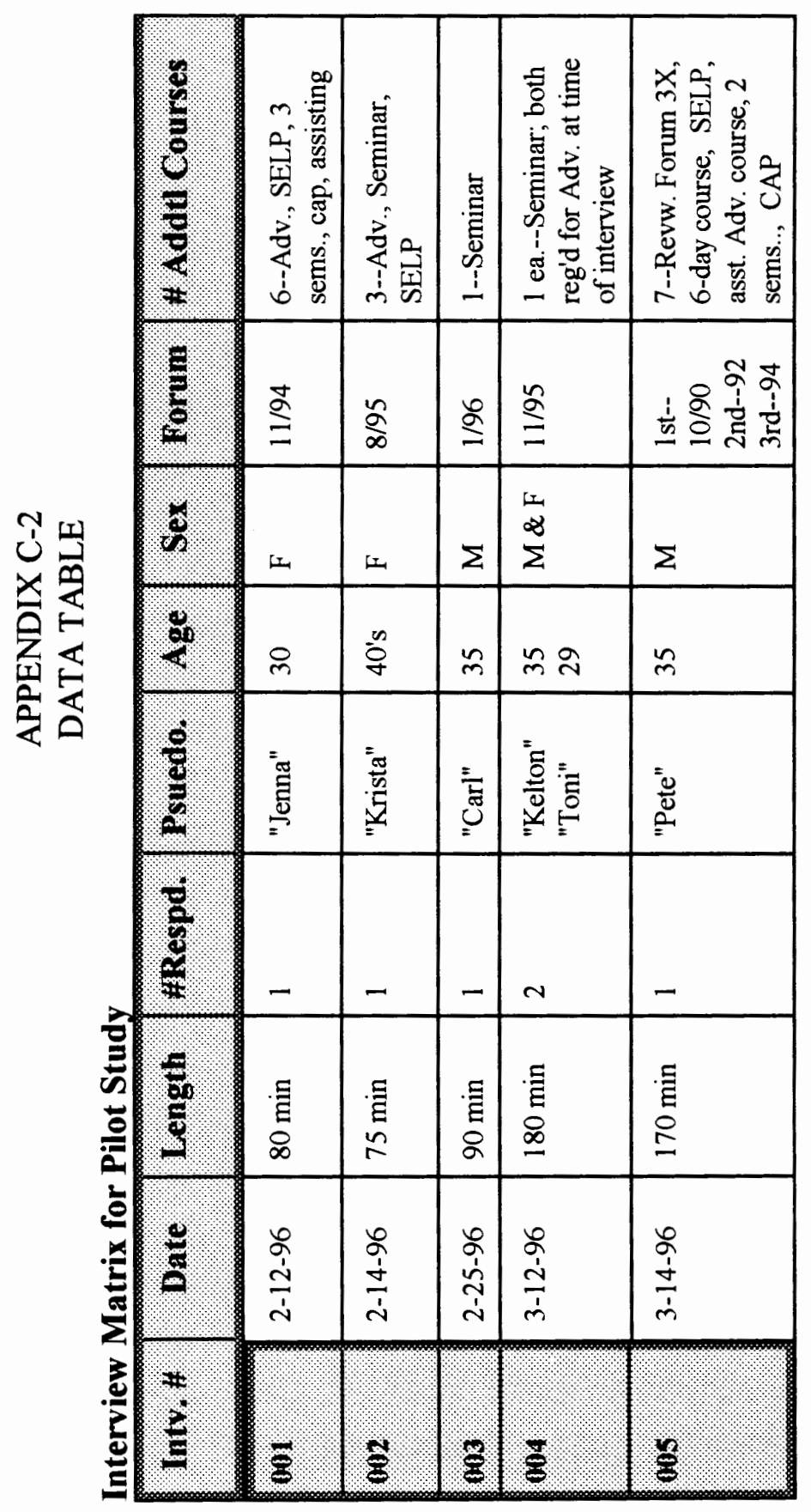

$\bar{\pi}$ 


\section{APPENDIX D}

\section{INFORMED CONSENT}

I, , hereby agree to participate in the research project investigating how people experience their participation with Landmark Education Corporation and translate that experience meaningfully in their everyday lives.

I understand that this study involves my verbally responding to open-ended questions asked by Debbie Gabbert, and that my time commitment to this study will be approximately 90 minutes. I understand that possible risks to me associated with this study are inconvenience and demand on my time for this interview. I may not receive any direct benefit from participation in this study, but my participation may help to increase knowledge which may benefit others in the future.

Debbie Gabbert has offered to answer any questions I may have about the study and what is expected of me in the study. I have been assured that my identity and information I give during the interviews will be kept confidential and that the names of all people in the study will be kept confidential. I understand that I am free to decline answering any question I do not feel comfortable answering or to withdraw from participation in this study at any time without jeopardizing my relationship with Debbie Gabbert or Portland State University.

This research project is in no way connected to the organization known as Landmark Education Corporation. The motivation for and results of this research are strictly for academic inquiry. While Landmark Education is aware that this research is being conducted and does not oppose it, they also do not support, endorse or acknowledge any connection to this research.

I have read and understand the foregoing information and agree to participate in this study.

Signature

Date:

If you experience any difficulties that are the result of your participation in this study, please contact the Chair of the Human Subjects Review Committee, Office of Grants and Contracts, 303 Cramer Hall, Portland State University, P.O. Box 751, Portland, OR 97207-0751, (503) 725-5262. If you have any questions about this study, please contact Debbie Gabbert at (206) 789-1182 or Susan Poulsen, PhD at (503) 725-3544. 


\section{APPENDIX E}

\section{INTERVIEW GUIDE}

1. When did you complete the Forum? (if more than once, find out how many and when)

2. How many and what courses have you participated in since then?

3. Tell me about your own decision to do the Forum. How did you decide to do the Forum?

4. What was (or tell me about) your experience of the Forum

Probes: What stands out for you about your experience in the Forum (or LEC's courses)? (for example, what distinctions stand out for you)

5. What has been the impact of LEC courses on your life? (or how has your participation in LEC courses shown up in your life)?

6. According to LEC, "transformation" is an outcome of participation in the Forum and other courses. What does the word "transformation" mean to you? Is that something you think you experienced or is that a word you would use to describe your experience with LEC courses?

7. What is your experience of the language or way of speaking associated with LEC?

8. I've noticed what seems to be a community or shared identity among people who have participated with LEC. What is your experience?

9. Does LEC or the courses you've particpated in come up in conversations with people?

Probes: Tell me about a recent conversation you had with someone about LEC. 


\title{
APPENDIX F
}

\section{ADDITIONAL SOURCES FOR INFORMATION}

\author{
ABOUT ERHARD, EST, AND LEC \\ Copyright 1992 Times Newspapers Limited : The \\ Times \\ July 22,1992 , Wednesday \\ SECTION: Home news \\ HEADLINE: New Age guru goes into hiding \\ BYLINE: Ben Macintyre \\ Copyright 1992 Times Newspapers Limited The \\ Times \\ July 22, 1992, Wednesday \\ SECTION: Home news \\ LENGTH: 987 words \\ HEADLINE: Mind game courses aimed at public \\ sector workers \\ BYLINE: Ray Clancy \\ Copyright 1992 The Washington Post The \\ Washington Post \\ July 7, 1992, Tuesday, Final Edition \\ SECTION: METRO; PAGE B3 \\ LENGTH: 641 words \\ HEADLINE: Self-Help Course Allegedly \\ Shattered a Life \\ SERIES: Occasional \\ BYLINE: Robert F. Howe, Washington Post Staff \\ Writer \\ Copyright 1991 Newsweek Newsweek \\ February 18, 1991, US Edition \\ SECTION: Life/Style; Mind; Pg. 72 \\ LENGTH: 919 words \\ HEADLINE: The Sorrows of Werner: For the \\ founder of est, a fresh round of charges \\ BYLINE: David Gelman with Pamel Abramson in \\ San Francisco and Elizabeth Ann Leonard in \\ New York \\ Copyright 1991 The Times Mirror Company Los \\ Angeles Times \\ December 29, 1991, Sunday, Home Edition \\ SECTION: Part A; Page 1; Column 1; Metro Desk \\ HEADLINE: Founder Of est targeted in campaign \\ by scientologists; Religion: \\ BYLINE: By Robert W. Welkos, Times Staff \\ Writer \\ Copyright 1991 The Atlanta Constitution The \\ Atlanta Journal and Constitution \\ February 17, 1991 \\ SECTION: National News; Section A; Page 21 \\ HEADLINE: Self-help guru Werner Erhard \\ selling empire \\ BYLINE: The San Francisco Chronicle \\ Copyright 1992 American Lawyer Media, L.P. \\ The Recorder \\ March 16, 1992, Monday \\ SECTION: Bar Talk; Pg. 2 \\ LENGTH: 353 words \\ HEADLINE: Cults: If You Can't Beat 'em ... \\ BYLINE: Stephen G. Hirsch \\ Copyright 1991 The Times Mirror Company Los \\ Angeles Times \\ March 27, 1991, Wednesday, Home Edition \\ SECTION: Business; Part D; Page 2; Column 2; \\ Financial Desk \\ HEADLINE: Guru ERHARD Accused of trying to \\ hide assests; Lawsuit: A Former Associate \\ Of the est program's founder alleges that he's \\ Copyright 1991 The Chronicle Publishing Co. The \\ San Francisco Chronicle \\ transferred property in an effort to evade creditors. \\ March 5, 1991, Tuesday, Final edition \\ BYLINE: By Martha Groves, Times Staff Writer \\ DATELINE: San Francisco
}

SECTION: NEWS; Pg. A9

LENGTH: 433 words

HEADLINE: More Allegations Against est

Founder 
Copyright (c) 1990 The New York Times Company; The New York Times

January 7, 1990, Sunday, Late Edition - Final SECTION: Section 7; Page 1, Column 1; Book

Review Desk

HEADLINE: Heidegger for Fun and Profit

BYLINE: By Anthony Gottlieb; Anthony Gottlieb, the science and technology editor of the British weekly The Economist, is a doctoral student in philosophy at University College, London.

Copyright 1990 The Chronicle Publishing Co. The San Francisco Chronicle APRIL 3, 1990, Tuesday, Final Edition SECTION: News; Pg. A4

HEADLINE: Ex-Employees Describe Abuse In Suit Against est's Erhard

BYLINE: Don Lattin, Chronicle Religion Writer

Copyright 1988 Crain Communications, Inc.; Crain's Chicago Business

December 5, 1988

SECTION: Rance Crain; Pg. 10

HEADLINE: Shining the light on hidden link BYLINE: By Rance Crain

Copyright (c) 1988 The Times Mirror Company; Los Angeles Times

December 2, 1988, Friday, Home Edition

SECTION: View; Part 5; Page 8; Column 1; View

Desk

HEADLINE: Transformation of est Founder

Werner Erhard; The noted salesman of personal

effectiveness is still attracting the buyers

BYLINE: By Iris Krasnow, United Press

International

DATELINE: Washington

Los Angeles Magazine Copyright Los Angeles

Magazine, Inc. 1988;

Business Dateline; Copyright (c) 1988 UMI/Data

Courier

May, 1988

SECTION: Vol 33; No 5; Sec 1; pg 106

HEADLINE: The Return of Werner Erhard: Guru

II

BYLINE: Mark MacNamara

DATELINE: San Francisco; CA; US
Vermont Business Magazine Copyright Manning

Publications Inc 1987;

Business Dateline; Copyright (c) 1987 UMI/Data

Courier

May, 1987

SECTION: Sec 1;pg 37

HEADLINE: New Age Workshops: Superlearning or Psychobabble?

BYLINE: William Sypher

DATELINE: Burlington; VT; US

Copyright (c) 1979 The Washington Post

April 14, 1979, Saturday, Final Edition

SECTION: Style; $\mathrm{Cl}$

HEADLINE: Encountering Werner Erhard;

Getting His Bearings by Moving West and

Heading est

Buckminster Fuller and the Head of est Engage in a Geodesic Dialogue

BYLINE: By Megan Rosenfeld

Copyright (c) 1977 The Washington Post

November 30, 1977, Wednesday, Final Edition

SECTION: Style; B1

HEADLINE: You Can Read This Article;

Read This Article in 10 Minutes and Improve

Your Life!;

BYLINE: By Michael Kernan

Copyright 1975 Forbes, Inc. Forbes

December 1, 1975

SECTION: FEATURES; Pg. 22

HEADLINE: The Power Of Positive Eyewash HIGHLIGHT: EST, the newest self-improvement medicine, is a mixture of eyewash and brainwash-- and yet many businessmen are buying it.

\section{Books about Werner Erhard and est:}

Werner Erhard: the transformation of a man, the founding of est.

Author: Bartley, William Warren

Publisher: Potter 1978

ISBN/ISSN: 0517535025

Call number B E67B 
Outrageous betrayal: the dark Journey of Werner Erhard, from est to exile Author: Pressman, Steven

Publisher: New York, St. Martin's Press, 1993 ISBN/ISSN: 0312092962

Call number B E679P

"60 Minutes and the Assassination of Werner Erhard"

Author: Jane Self, Ph. D,

Publisher: Breakthru Publications

IBSN/ISSN: 0-942540-23-9

Call number:

est; 60 hours that transform your life

Author: Bry, Adelaide

Publisher: Harper 1976

Call number 616.8 B915E

Getting it: the psychology of est

Author: Fenwick, Sheridan

Publisher: Lippincott 1976

ISBN/ISSN: 0397011709

Call number 158.F343G

The Book of EST

Author: Rhinehart, Luke

Publisher: Holt 1976

ISBN/ISSN: 0-03-018326-X

Call number 158.R473B
Some more current articles related to

LEC:Capuzzo, J.P. (1996, March 16). Come on! There's a new life waiting over the weekend.

Philidephia Inquirer.

Capuzzo, J.P. (1996, March 22). All types of workshops for plumbing the sould.

Philidephia Inquirer.

Walker, S. (1993, September). On course for a change. Health \& Fitness Magazine.

The weekend that changed my life. (1997, January). Teen Magazine.

Emmerich, W. (1994, May 26). Landmark forum can make a difference. Northside Sun.

Pratt, C. (1995, July) earning his keep, neatly. The New York Times.

Klausner, H. (1993, October) Globetrotting volunteer brings hope to the blind. The New York Times. 